Linköping University Medical Dissertation No. 1579

\title{
Neuropeptide Receptors as Treatment Targets in Alcohol Use Disorders
}

\author{
Abdul Maruf Asif Aziz
}

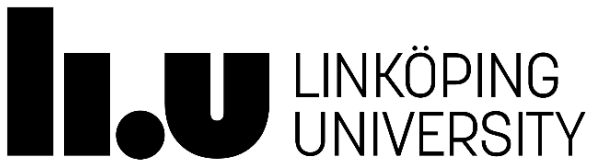

Center for Social and Affective Neuroscience (CSAN)

Department of Clinical and Experimental Medicine (IKE) Linköping University, Sweden 
(C) Abdul Maruf Asif Aziz, 2017

Cover illustration by Dr. Shadi Jafari.

Published articles have been reprinted with the permission of the copyright holders.

Printed by LiU-Tryck, Campus Valla, Linköping, Sweden.

ISBN: 978-91-7685-484-6.

ISSN: 0345-0082.

Series: Linköping University Medical Dissertation No.1579; 15 Sep 2017. 


\section{Neuropeptide Receptors as Treatment Targets in Alcohol Use Disorder}

\section{Thesis for doctoral degree (Ph.D) by Abdul Maruf Asif Aziz}

\section{Principal Supervisor:}

Docent Annika Thorsell, Ph.D

Department of Clinical and Experimental Medicine (IKE)

Linköping University, Sweden

\section{Co-supervisor:}

Docent David Engblom, Ph.D

Department of Clinical and Experimental Medicine (IKE)

Linköping University, Sweden

\section{Opponent:}

Docent Elizabeth Jerlhag Holm, Ph.D

Department of Pharmacology

University of Gothenburg, Sweden

\section{Examination board:}

Professor Giannis Spyrou, Ph.D

Department of Clinical and Experimental Medicine (IKE)

Linköping University, Sweden

Docent Jakob Ström, Ph.D

Department of Clinical and Experimental Medicine (IKE)

Linköping University, Sweden

Docent Erika Roman, Ph.D

Institute of Pharmaceutical Life Sciences

Uppsala University, Sweden

Mattias Alenius, Ph.D (Universitetslektor)

Department of Clinical and Experimental Medicine (IKE)

Linköping University, Sweden 


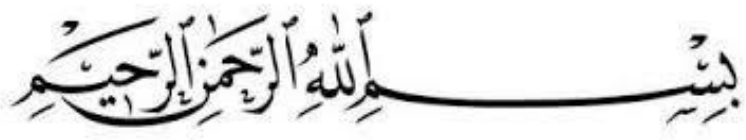

In the Name of Allāh, the Most Gracious, the Most Merciful

Dedicated to my beloved \& respected parents...

"My greatest strength is the love for my people, my greatest weakness is that I love them too much." 


\section{ABSTRACT}

Alcohol use disorder (AUD) is a complex disorder with multiple pathophysiological processes contributing to the initiation, progression and development of the disease state. AUD is a chronic relapsing disease with escalation of alcohol-intake over time in repeated cycles of tolerance, abstinence and relapse and hence, it is very difficult to treat. There are only a few currently available treatments with narrow efficacy and variable patient response. Thus it is important to find new, more effective medications to increase the number of patients who can benefit from pharmacological treatment of AUD.

The research presented in this thesis work focuses on the critical involvement of central neuropeptides in alcohol-related behaviors. The overall aim was to evaluate the nociceptin/orphanin FQ (NOP) receptor, the neuropeptide Y (NPY) Y2 receptor and the melanin-concentrating hormone $(\mathrm{MCH})$ receptor 1 as novel and potential pharmacological treatment targets for AUD by testing the NOP receptor agonist SR8993, the NPY-Y2 receptor antagonist CYM-9840 and the $\mathrm{MCH} 1$ receptor antagonist GW803430 in established animal models.

In the first study (Paper I), the novel and selective NOP agonist SR-8993 was assessed in rat models of motivation to obtain alcohol and relapse to alcohol-seeking behavior using the operant self-administration (SA) paradigm. Firstly, treatment with SR-8993 $(1 \mathrm{mg} / \mathrm{kg})$ showed a mildly anxiolytic effect and reversed acute alcohol withdrawal-induced "hangover" anxiety in the elevated plus-maze (EPM). Next, it potently attenuated alcohol SA and motivation to obtain alcohol in the progressive ratio responding (PRR) and reduced both alcohol cue-induced and yohimbine stressinduced reinstatement of alcohol seeking, without affecting the pharmacology and metabolism of alcohol nor other control behaviors. To extend these findings, SR-8993 was evaluated in escalated alcohol-intake in rats. Treatment with SR-8993 significantly suppressed alcohol-intake and preference in rats that were trained to consume high amounts of alcohol in the two-bottle free choice intermittent access (IA) paradigm. SR8993 also blocked operant SA of alcohol in rats that showed robust escalation in operant alcohol SA following chronic IA exposure to alcohol.

In the second study (Paper II), SR-8993 was further evaluated in a model for escalated alcohol-intake induced by long-term IA exposure to alcohol. The effect of previous experience on operant alcohol SA on two-bottle free choice preference drinking was evaluated and sensitivity to treatment with SR-8993 was tested in rats selected for escalated and non-escalated alcohol seeking behavior. We found that rats exposed to the combined SA-IA paradigm showed greater sensitivity to SR-8993 treatment. In addition, acute escalation of alcohol SA after a three-week period of abstinence was completely abolished by pretreatment with SR-8993.

In the third study (Paper III), the effects of the novel, small molecule NPY-Y2 antagonist CYM-9840 were tested in operant alcohol SA, PRR which is a model for 
motivation to work for alcohol and reinstatement of alcohol-seeking behavior paradigm. Treatment with CYM-9840 (10 mg/kg) potently attenuated alcohol SA, progressive ratio responding and stress-induced reinstatement using yohimbine as the stressor, while alcohol cue-induced reinstatement was unaffected. Moreover, a range of control behaviors including taste sensitivity, locomotor and pharmacological sensitivity to the sedative effects of alcohol remained unaffected by CYM-9840 pretreatment, indicating that its effects are specific to the rewarding and motivational aspects of alcohol-intake and related behaviors. CYM-9840 also reversed acute alcohol withdrawal-induced "hangover" anxiety measured in the EPM and reduced alcohol-intake in the 4 hour limited access two-bottle free choice preference drinking model.

Finally, in the fourth study (Paper IV), the selective MCH1-R antagonist GW803430 was tested in rat models of escalated alcohol-intake. Pretreatment with GW803430 (effective at $10 \& 30 \mathrm{mg} / \mathrm{kg}$ ) dose-dependently reduced alcohol and food-intake in rats that consumed high amounts of alcohol during IA, while it only decreased food-intake in rats that consumed low amounts of alcohol during IA, likely due to a floor effect. Upon protracted abstinence following IA, GW803430 significantly reduced operant alcohol SA and this was associated with adaptations in $\mathrm{MCH}$ and $\mathrm{MCH} 1-\mathrm{R}$ geneexpression. In contrast, GW803430 did not affect escalated alcohol SA induced by chronic alcohol vapor exposure and this was accompanied by no change in $\mathrm{MCH}$ or $\mathrm{MCH} 1-\mathrm{R}$ gene expression. Overall, these results suggest that the MCH1-R antagonist affects alcohol-intake through regulation of both motivation for caloric-intake and the rewarding properties of alcohol.

In conclusion, our results suggest critical roles for these central neuropeptides in the regulation of anxiety and of alcohol reward, making them potential pharmacological targets in the treatment of AUD. 


\section{POPULÄRVETENSKAPLIG SAMMANFATTNING}

Bruk av alkohol är vanligt förekommande och socialt accepterat i stora delar av världen. Detta trots den skada som alkoholbruk medför, både för individen själv, familj och vänner samt samhället i stort. Enligt Världshälsoorganisationen, WHO, står riskbruk av alkohol för cirka $6 \%$ av den årliga dödligheten och är en av orsakerna till runt 200 sjukdomstillstånd ${ }^{1}$.

Alkoholberoende utvecklas gradvis över tid. Innan beroendet utvecklats talar man om riskbruk, dvs ett konsumtionsmönster som är skadligt för individen, vilket leder till en obalans i olika kemiska ämnen i hjärnan. Dessa påverkar, bland annat, omdöme, positiva känslor ("belöning") och impulskontroll. När detta pågått en tid väljer individen att dricka alkohol inte för belöningskänslan utan för att må bättre eller glömma negativa känslor och upplevelser. Orsaken till ett alkoholberoende är en kombination av flera olika riskfaktorer, inkluderande både miljö-faktorer och genetik.

Trots den höga sjukdomsbördan och att man vet hur varierande orsakerna till ett alkoholberoende kan vara, finns idag ett mycket begränsat antal läkemedel. Dessa inkluderar disulfiram (antabus), naltrexon (ReVia) och akamprosat (Campral). Målet med den här beskrivna forskningen är att ta fram flera läkemedel för att bättre behandla individer med olika sjukdomsprofil.

I denna doktorand avhandling studeras tre av hjärnans signalsystem och hur reglering av deras funktion påverkar beteenden som alkohol-intag, återfallsrisk, belöningsupplevelse och impulskontroll. De tre signalsystem som studeras tillhör alla en grupp, de är alla neuropeptider och de är: nociceptin/orfanin FQ (NOP), neuropeptid Y (NPY) och melanin koncentrerande hormon (MCH). NOP är släkt med opioider (t.ex. morfin), finns $i$ hela nervsystemet och deltar bland annat i reglering av smärta, inlärning och ångest. NPY reglerar matintag, fetma, stressvar och liknande funktioner och kan påvisas även den i hela nervsystemet. $\mathrm{MCH}$ däremot kan bara ses i specifika hjärndelar och den reglerar energibalans, dygnsrytm, födointag $\mathrm{mm}$.

Gemensamt för effekter av ändring av funktion hos dessa tre var att intag av alkohol signifikant minskades, risken att återfalla till att konsumera alkohol försvagades och motivationen att vilja ha alkohol minskades. Dessutom visas att funktionen hos två av signalsystemen, NOP och $\mathrm{MCH}$, påverkas av en längre tids alkoholkonsumtion. Detta påvisas genom att känsligheten för funktionsändring är större efter högt intag av alkohol. Sammantaget illustrerar de i avhandlingen ingående studierna att neuropeptid-system i hjärnan kan vara attraktiva för framtagande av framtida läkemedel vilka kan hjälpa individer med alkohol-riskbruk eller beroende².

\footnotetext{
1Källa: http://www.who.int/mediacentre/factsheets/fs349/en/ (20170803)

${ }^{2}$ Avhandling: Neuropeptide Receptors as Treatment Targets in Alcohol Use Disorder; Författare: Abdul Maruf Asif Aziz, LiU avhandling \# 1579, 15 September 2017
} 


\section{LIST OF SCIENTIFIC ARTICLES}

I. The nociceptin/orphanin FQ receptor agonist $\mathrm{SR}-8993$ as a candidate therapeutic for alcohol use disorder: validation in rat models

Aziz AMA, Brothers S, Sartor G, Holm L, Heilig M, Wahlestedt C, Thorsell A.

Psychopharmacology (Berl). 2016 Oct;233(19-20):3553-63. doi: 10.1007/s00213016-4385-8. PMID: 27515665.

II. Escalation of self-administration following intermittent access to alcohol selects for sensitivity to treatment with a nociceptin/orphanin FQ agonist SR8993

Aziz AMA, Karlsson SC, Holm L, Ahmed A, Brothers S, Sartor G, Heilig M, Wahlestedt C, Thorsell A.

Manuscript to be submitted to the journal of Alcoholism: Clinical and Experimental Research.

III. The neuropeptide Y-Y2 receptor antagonist CYM-9840 as a putative treatment target in alcohol use disorder: validation in rat models

Aziz AMA, Brothers S, Holm L, Sartor G, Heilig M, Wahlestedt C, Thorsell A.

Manuscript to be submitted to the journal of Psychopharmacology.

IV. Melanin-Concentrating Hormone and Its $\mathrm{MCH}-1$ Receptor: Relationship Between Effects on Alcohol and Caloric-intake

Karlsson C, Aziz AMA, Rehman F, Pitcairn C, Barchiesi R, Barbier E, Wendel Hansen M, Gehlert D, Steensland P, Heilig M, Thorsell A.

Alcoholism: Clinical Experimental Research. 2016 Oct;40(10):2199-2207. doi: 10.1111/acer.13181. PMID: 27579857. 


\section{TABLE OF CONTENTS}

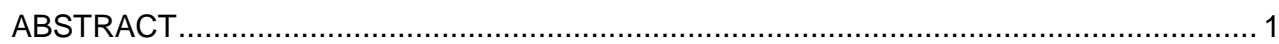

POPULÄRVETENSKAPLIG SAMMANFATTNING ................................................ 3

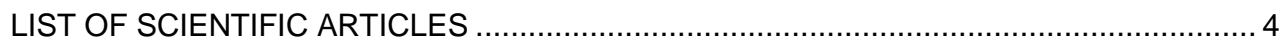

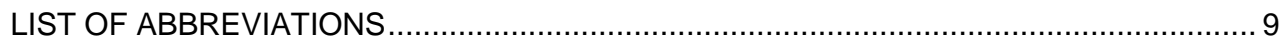

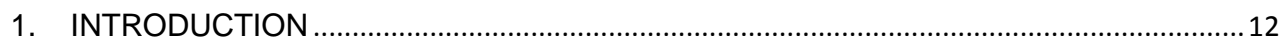

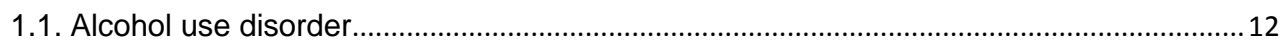

1.1.1. Diagnostic criteria for alcohol use disorder .............................................. 13

1.1.2. Alcohol use disorder criteria according to DSM-V ...................................... 14

1.1.3. Transition from controlled alcohol use to alcohol use disorder ........................ 14

1.1.4. Risk factors for alcohol use disorder ........................................................ 16

1.1.5. Anxiety and depression are closely associated with alcohol use disorder ....... 16

1.1.6. Neurobiological effects of alcohol ......................................................... 17

1.1.7. Current treatment options for alcohol use disorder .................................... 18

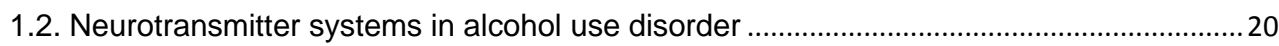

1.2.1. The GABAergic system in alcohol use disorder ........................................ 21

1.2.2. The glutamatergic system in alcohol use disorder ..................................... 21

1.2.3. The dopaminergic system in alcohol use disorder ..................................... 22

1.2.4. The opioid system in alcohol use disorder ................................................ 23

1.2.5. The serotonergic system in alcohol use disorder ......................................... 24

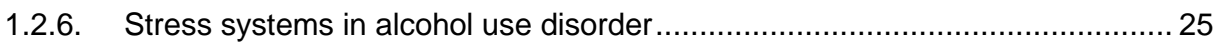

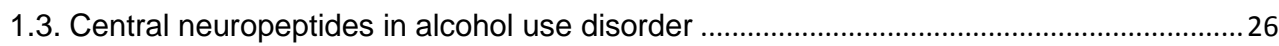

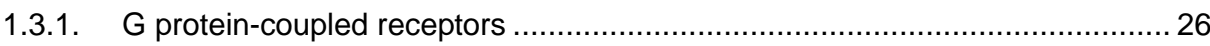

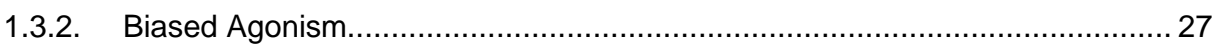

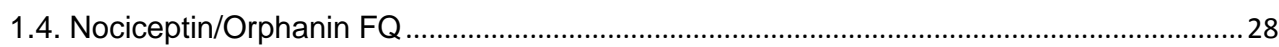

1.4.1. Tissue distribution of the nociceptin/orphanin FQ receptor ............................ 28

1.4.2. Functional role of nociceptin/orphanin FQ ............................................... 29

1.4.3. The role of nociceptin/orphanin FQ system in reward processing ................... 30

1.4.4. The nociceptin/orphanin FQ system in alcohol use disorder ......................... 30

1.4.5. Buprenorphine in the rewarding effects of morphine and alcohol.................. 31

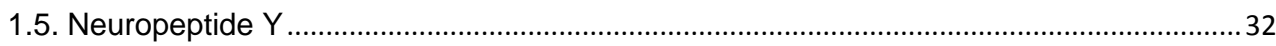

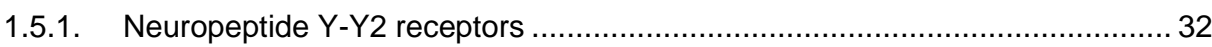

1.5.2. Neuropeptide $Y$ in the modulation of food and alcohol-intake ....................... 33

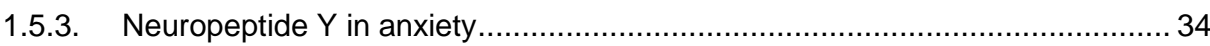

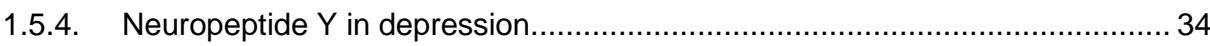


1.6. Melanin-concentrating hormone. .36

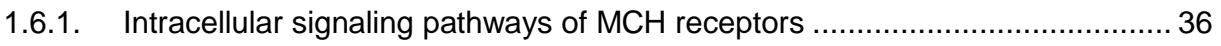

1.6.2. $\mathrm{MCH}$ in modulation of food-intake and energy homeostasis ........................... 37

1.6.3. Shared mechanism in the regulation of food and drug reward ......................... 37

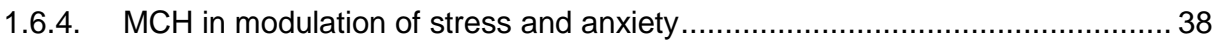

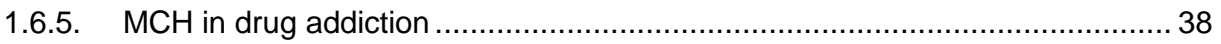

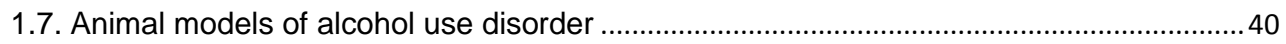

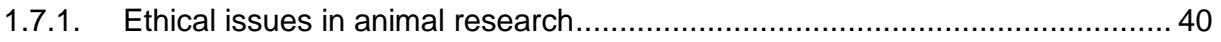

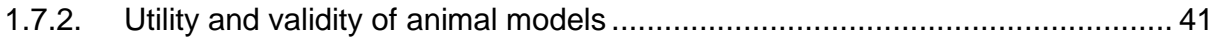

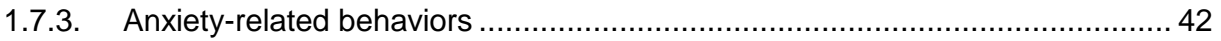

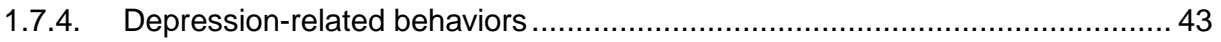

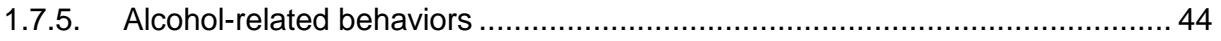

1.7.6. Compulsive alcohol seeking and related behaviors .................................... 45

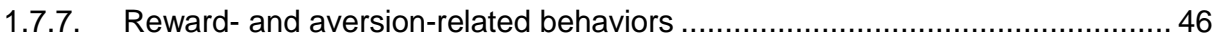

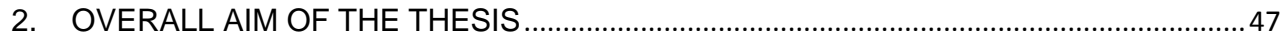

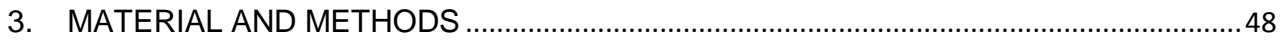

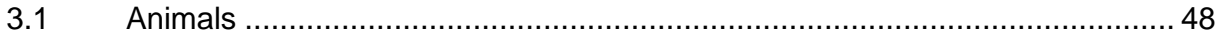

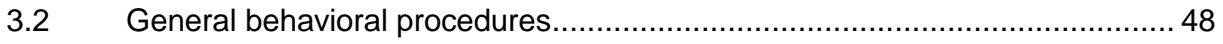

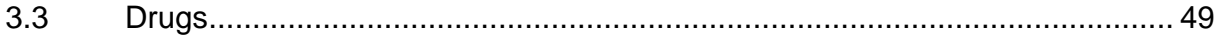

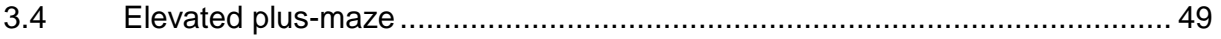

3.5 Acute alcohol withdrawal-induced anxiety ............................................. 50

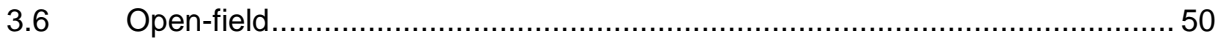

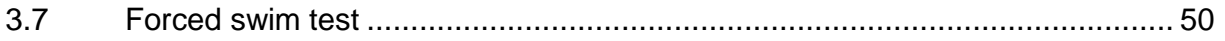

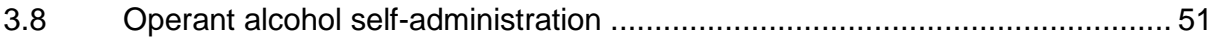

3.9 Progressive ratio responding for alcohol self-administration .........................51

3.10 Cue- and stress-induced reinstatement of alcohol seeking .......................... 52

3.11 Two-bottle free choice drinking (4 hours limited access) .............................. 52

3.12 Two-bottle free choice drinking (Intermittent Access) .................................. 52

3.13 Operant alcohol self-administration following intermittent ............................. 53

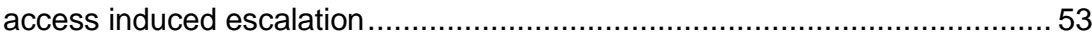

3.14 Operant alcohol self-administration after intermittent alcohol .........................53

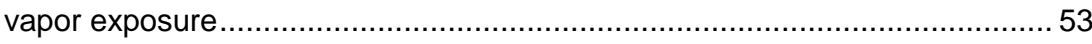

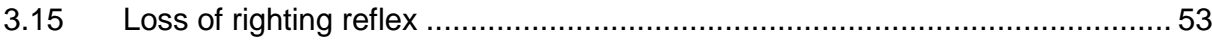

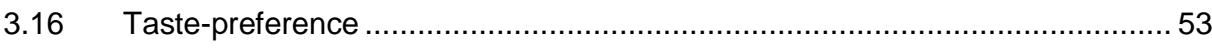

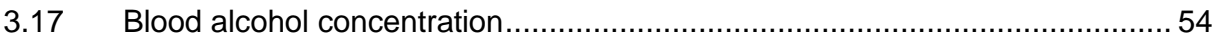




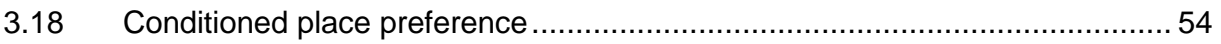

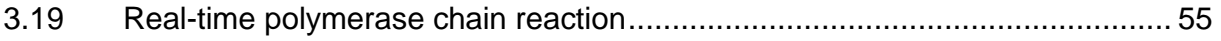

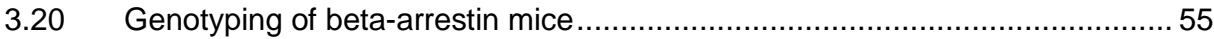

3.21 Immunohistochemistry of extracellular signal-regulated...............................56

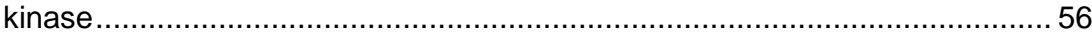

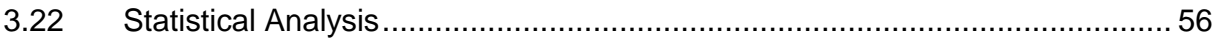

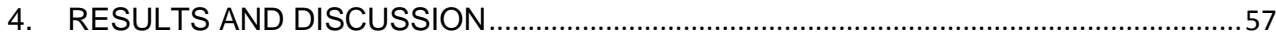

4.1 The nociceptin/orphanin FQ receptor agonist SR-8993 as a candidate therapeutic for alcohol use disorders: validation in rat models (Paper I).................................................5

4.1.1 Acute alcohol withdrawal-induced anxiety in the elevated plus-maze was reversed by SR-8993.

4.1.2 Operant self-administration, progressive ratio responding and cue- and stressinduced reinstatement of alcohol seeking were attenuated by SR-8993 in alcohol naïve Wistar rats

4.1.3 Alcohol-intake in the 4 hour limited access and intermittent access drinking was reduced by SR-8993

4.1.4 SR-8993 reduced escalated alcohol-intake in intermittent access two-bottle free choice drinking and operant self-administration in rats with a history of escalation

4.1.5 Control experiments confirmed that the effects of SR-8993 were specific to alcohol reward and motivation to seek alcohol

4.2 Escalation of self-administration following intermittent access to alcohol selects for sensitivity to treatment with a nociceptin/orphanin FQ receptor agonist SR-8993 (PAPER II)

4.2.1 A history of operant self-administration resulted in increased alcohol-intake and preference in the intermittent access two-bottle free choice model and escalated operant responding.

4.2.2 A robust positive correlation between alcohol-intake and preference regardless of previous experience with alcohol self-administration. 62

4.2.3 Intermittent access to alcohol resulted in escalated alcohol self-administration and increased motivation to obtain alcohol

4.2.4 Intermittent access to voluntary alcohol drinking resulted in a pronounced escalation of operant alcohol self-administration in a subgroup of individuals. 63

4.2.5 SR-8993 selectively suppressed alcohol-intake in individuals with escalated self-administration

4.2.6 SR-8993 potently suppressed abstinence-induced alcohol self-administration 64

4.3 The neuropeptide $\mathrm{Y}-\mathrm{Y} 2$ antagonist as a putative treatment target in alcohol use disorder: validation in rat models (Paper III)

4.3.1 CYM-9840 prevented acute alcohol-induced withdrawal anxiety 65 
4.3.2 CYM-9840 reduced alcohol-intake in the two-bottle free choice preference drinking with 4 hour limited access

4.3.3 CYM-9840 suppressed operant self-administration of alcohol, motivation to seek alcohol and stress- but not cue-induced reinstatement of alcohol seeking

4.3.4 Control experiments confirmed the behavioral specificity of the effects of CYM9840 on alcohol-related behaviors

4.4 Melanin-concentrating hormone and its $\mathrm{MCH} 1$ receptor: Relationship between effects on alcohol and caloric intake (Paper IV)

4.4.1 Intermittent Access to alcohol resulted in robust escalation of intake that was accompanied by a caloric shift from food to alcohol.....

4.4.2 GW803430 reduced caloric-intake from both alcohol and food in the high drinker group only.....

4.4.3 Escalated alcohol self-administration induced by intermittent access was suppressed by GW803430 administration during protracted abstinence following intermittent access.

4.4.4 Escalated alcohol self-administration induced by chronic alcohol vapor exposure was not suppressed by GW803430 administration during acute withdrawal

4.4.5 Expression of $\mathrm{MCH}$ and $\mathrm{MCH} 1-\mathrm{R}$ is dysregulated in the hypothalamus and nucleus accumbens during protracted abstinence from intermittent access but not during acute withdrawal from alcohol vapor ...................................... 71

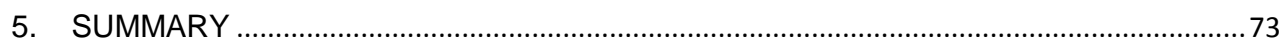

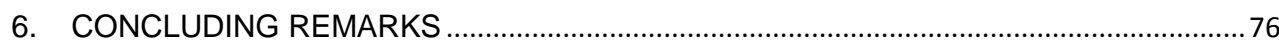

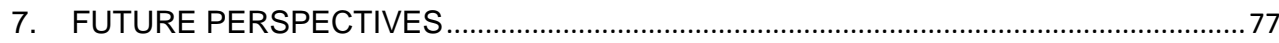

7.1 SR-8993 blocked expression of alcohol-induced CPP both at ...................... 78

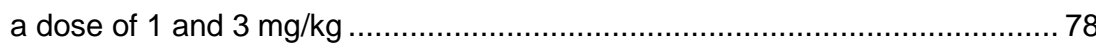

7.2 SR-8993 blocked alcohol-induced CPP via a beta-arrestin .......................... 79

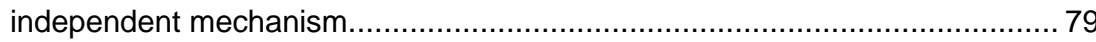

7.3 Pretreatment with SR-8993 attenuates acute alcohol-induced....................... 80 phosphorylation of ERK in key brain regions ........................................... 80

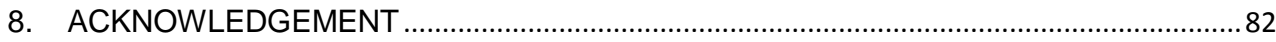

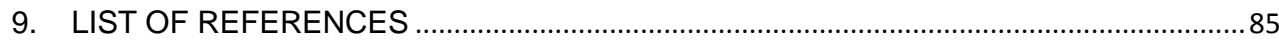




\section{LIST OF ABBREVIATIONS}

ACTH

ADE

ALDH

AMPA

AUD

BAC

BLA

BNST

Camp

CeA

CNS

CPA

CPP

CRH

CSF

DALYs

DARPP32

Adrenocorticotropic hormone

Alcohol deprivation effect

Alcohol dehydrogenase

a-amino-3-hydroxy-5-methyl-4-isoxazolepropionic acid

Alcohol use disorder

Blood alcohol concentration

Basolateral amygdala

Bed nucleus of the stria terminalis

Cyclic adenosine monophosphate

Central Amygdala

Central nervous system

Conditioned place aversion

Conditioned place preference

Corticotropin releasing hormone

Cerebrospinal fluid

Disability adjusted life years

Dopamine and cyclic adenosine monophosphate phosphoprotein $32 \mathrm{kDa}$

DOR

Delta opioid receptor

DSM

Diagnostic and statistical manual

DYN

Dynorphin

EAAT

Excitatory amino acid transporters

ECT

Electroconvulsive therapy

EPM

Elevated plus-maze

ERK

Extracellular signal-regulated kinase

FDA

Food and Drug Administration

fMRI

Functional magnetic resonance imaging

FST

Forced swim test 


\begin{tabular}{|c|c|}
\hline GABA & Y-aminobutyric acid \\
\hline GDP & Guanosine diphosphate \\
\hline GEF & Guanine nucleotide exchange factor \\
\hline GPCRs & G-protein coupled receptors \\
\hline GTP & Guanosine triphosphate \\
\hline HPA & Hypothalamic pituitary adrenal \\
\hline IA & Intermittent access \\
\hline ICD & $\begin{array}{l}\text { International statistical classification of diseases and related } \\
\text { health problems }\end{array}$ \\
\hline ICV & Intracerebroventricular \\
\hline IHC & Immunohistochemistry \\
\hline KOR & Kappa opioid receptor \\
\hline LGICs & Ligand gated ion channels \\
\hline LH & Lateral hypothalamus \\
\hline LORR & Loss of righting reflex \\
\hline MAPK & Mitogen-activated protein kinase \\
\hline $\mathrm{MCH}$ & Melanin-concentrating hormone \\
\hline MCH1-R & Melanin-concentrating hormone 1 receptor \\
\hline MCH2-R & Melanin-concentrating hormone 2 receptor \\
\hline mPFC & Medial prefrontal cortex \\
\hline NAc & Nucleus accumbens \\
\hline NEI & Neuropeptide glutamic acid isoleucinamide \\
\hline NGE & Glycine glutamic acid \\
\hline NMDA & $\mathrm{N}$-methyl-D-aspartate \\
\hline NOP & Nociceptin/orphanin FQ \\
\hline NP & Non-preferring rats \\
\hline NPY & Neuropeptide $Y$ \\
\hline OPRM & Mu opioid receptor 1 \\
\hline ORL1 & Opioid receptor like-1 \\
\hline $\mathbf{P}$ & Preferring rats \\
\hline
\end{tabular}




$\begin{array}{ll}\text { PET } & \text { Positron emission tomography } \\ \text { PFC } & \text { Prefrontal cortex } \\ \text { POMC } & \text { Proopiomelanocortin } \\ \text { PRR } & \text { Progressive ratio responding } \\ \text { SA } & \text { Self-administration } \\ \text { SDS } & \text { Social defeat stress } \\ \text { SNP } & \text { Single nucleotide polymorphism } \\ \text { SSRI } & \text { Selective serotonin reuptake inhibitor } \\ \text { VDCCs } & \text { Voltage-dependent calcium channels } \\ \text { VTA } & \text { Ventral tegmental area } \\ \text { WHO } & \text { World Health Organization } \\ \text { 5HT } & \text { Serotonin }\end{array}$




\section{INTRODUCTION}

\subsection{Alcohol use disorder}

Alcohol is currently one of the most widely used and abused drugs in the world. Alcohol-related accidents, cognitive disorders and other problems caused by alcohol abuse rank high as causes of societal disability burden and result in tremendous human suffering (1-3). Alcohol use disorder (AUD) is among the most prevalent, difficult to treat and undertreated mental disorders in developed countries (4). It is well known that alcohol at lower doses is consumed for its rewarding, anxiolytic and social facilitation effects. With increasing doses, it induces cognitive and motor impairment and disruption of emotional stability that increases the likelihood of injury. Heavy alcohol drinking can be a casual factor in various chronic diseases, including cancer, cardiovascular disease, diabetes, gastrointestinal disease, neuropsychiatric disorders and infectious disease(5). This leads to increased healthcare costs (4).

Individuals with AUD have significantly impaired control over their alcohol-intake and maintain their drinking patterns despite detrimental effects on their health and their relationships with their spouse, children, family members, friends and coworkers (5). Moreover, chronic intoxication is associated with increased risk taking and impulsivity that can result in catastrophic events such as automobile accidents that cause harm to others. This along with decreased productivity and increased criminality, contributes to the devastating societal and economic consequences of AUD (6).

The World Health Organization (WHO) has reported that alcohol directly or indirectly causes approximately 3.3 million deaths per year, which is $5.9 \%$ of all deaths globally. AUD also contributes $5.1 \%$ to the global disease burden, which is calculated as disability-adjusted life years lost (DALYs). WHO has also reported that around 2 billion people worldwide consume alcohol, $5-10 \%$ of whom will eventually escalate their use and progress to develop dependence (5). The progression of alcohol dependence is due to a combination of biological, psychiatric and social processes (6). Genetic heritability contributes approximately $50-60 \%$ of the risk for developing AUD, however, the contribution is highly polygenic. Interactions between genetic and environmental factors determine the risk for addiction (7). Alcohol-associated cues such as peers, cultural factors and social settings contribute to this, as does exposure to alcohol itself. Other environmental factors such as stress and trauma can have detrimental effects that increase the vulnerability of developing $\operatorname{AUD}(10,11)$.

AUD is a complex state with diverse pathophysiological processes. This results in a high degree of diversity within the patient population. Because of this, patient response to the few pharmacotherapies that are currently available is quite variable. Therefore, one of the most important challenges in this research area is to find new, more effective medications, thereby increasing the treatable portion of the patient population. Basic neuroscience research can improve understanding of AUD 
pathophysiology and in doing so can identify novel mechanisms that can be targeted by more effective pharmacotherapies.

\subsubsection{Diagnostic criteria for alcohol use disorder}

Two main diagnostic manuals exist that are used to assess alcohol dependence. The Diagnostic and Statistical Manual of Mental Disorders (DSM) classification system is published by the American Psychiatric Association and is widely accepted as a standard for diagnosing AUD in the USA and many other countries. The International Statistical classification of Diseases and Related Health Problems (ICD) is published by the $\mathrm{WHO}$ and describes diagnostic criteria for a wide range of conditions (8).

The $5^{\text {th }}$ edition of the DSM (DSM-V) introduced some major changes to previous diagnostic criteria for AUD. The current criteria as stated in DSM-V are grouped as outlined below:

Criteria 1-4: These describe drinking habits, total amount of consumption, timing of drinking and willingness to drink or craving for alcohol. The criterion describing craving for alcohol is a new addition.

Criteria 5-7: These describe drinking more than intended resulting in disrupted attention at work, school and in the home. The individual may isolate themselves from social and recreational activities and prefer drinking to these activities. The individual may be willing to give up personal hobbies in order to drink alcohol which ultimately decreases general motivation.

Criteria 8-9: These describe risk-taking behavior. The individual will take physical risks or behave violently due to the effects of alcohol. The individual may drive a car or swim unsafely while drinking and not be concerned about the detrimental on their own and others' safety.

Criteria 10-11: These are based on the development of tolerance and withdrawal symptoms. When tolerance develops to alcohol, the amount of alcohol required to elicit the desired effect increases over time. The time course to develop tolerance can vary based on factors such as race, gender, age, ethnicity and body size and composition which make it complex to determine the development of tolerance in an individual patient. Alcohol withdrawal occurs with unplanned or sudden cessation of alcohol drinking. Symptoms include anxiety, tremor, depression, fatigue, sweating and various cognitive disorders are among others. These highly negative and aversive withdrawal effects often drive the individual to resume alcohol consumption to eliminate the unpleasant feelings they cause (9).

In the current DSM-V for the diagnosis of AUD the individual must meet at least two criteria out of eleven (specified on the next page). Based on the number of the criteria met, the severity of the AUD is further classified into three categories; two to 
three symptoms is diagnosed as mild AUD, four to five as moderate AUD and more than five as severe AUD.

\subsubsection{Alcohol use disorder criteria according to DSM-V}

1. The amount of alcohol-intake is higher than expected and consumption occurs over a longer period of time.

2. The individual wants to cut down or cease alcohol drinking but is unable to do so.

3. Spending a lot of time drinking, obtaining alcohol and recovering from the effects of alcohol.

4. Craving or a strong desire to drink alcohol.

5. Repeated alcohol drinking results in failure to meet obligations at work, school and home.

6. Continued high level of alcohol drinking although it creates social and interpersonal problems.

7. Giving up and becoming isolated from social, occupational and recreational activities.

8. Continued alcohol drinking in unsafe situations such as driving, swimming or using machinery.

9. Unable to discontinue or reduce alcohol drinking when it is causing or exacerbating physical or psychological conditions such as liver disease, cancer, cardiovascular disease, anemia, dementia and depression.

10. Needing to drink much more than before to get the same effect or experiencing reduced effects with the same level of drinking (tolerance).

11.Withdrawal symptoms such as sleep disturbance, anxiety, depression, agitation, confusion or hallucinations that occur when ceasing alcohol consumption and can only be resolved by consuming more alcohol.

\subsubsection{Transition from controlled alcohol use to alcohol use disorder}

At lower doses and in the early stages of the addiction process, drinking to intoxication is mainly driven by the positive reinforcing effects of alcohol and individuals are motivated to consume alcohol primarily for its rewarding effects $(7,10)$. Thus, alcohol consumption leading to intoxication is initially driven by the craving for alcohol reward. If this process continues it may lead to repeated episodes of heavy drinking followed 
by abstinence (Figure 1). As this occurs, the individual will associate environmental stimuli such as social context, specific places and specific other people with alcohol use (10). This is a critical component in the development of addiction. As the addiction progresses the cues previously associated with alcohol use will produce conditioned craving for the rewarding effects of alcohol and will gain the ability to induce relapse to alcohol seeking (11). This process of contextual cues driving drug-seeking behavior after a period of abstinence is called relapse in humans and reinstatement in animal models of addiction $(12,13)$.

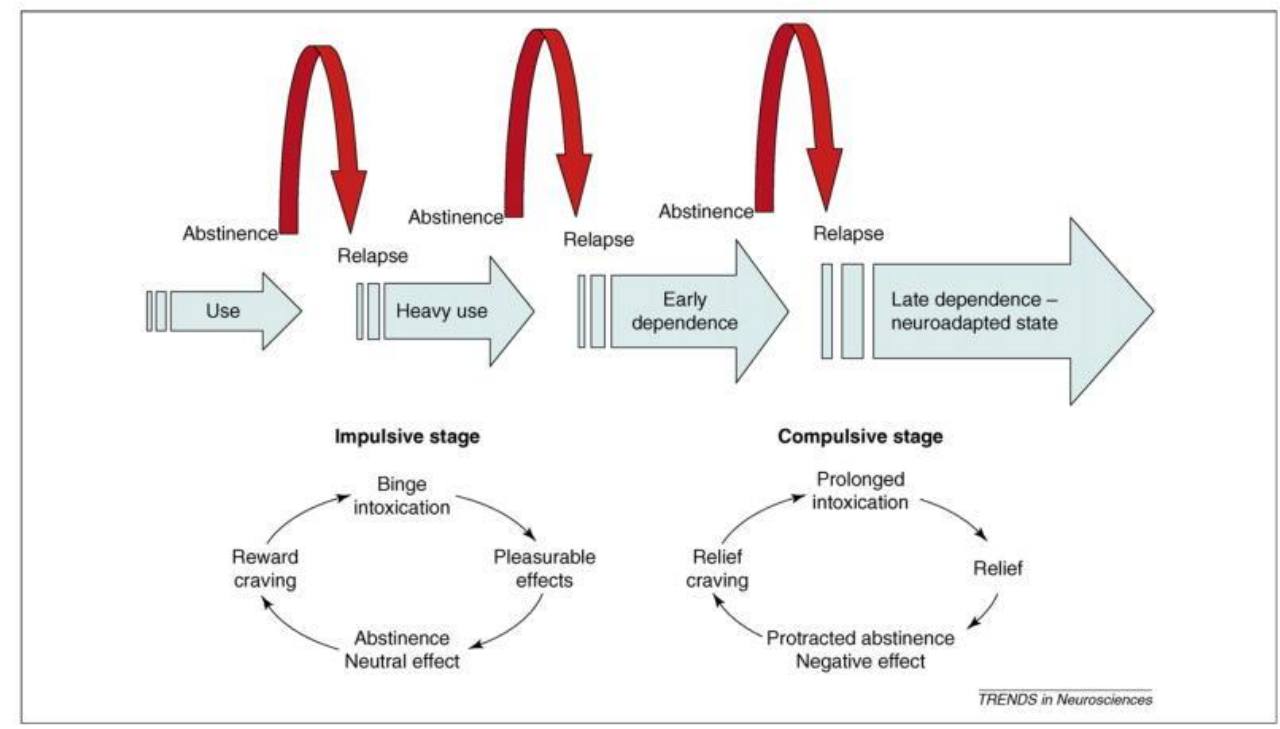

Figure 1. A schematic representation of the progression and transition to alcohol dependence over time. At the early impulsive stage the use of alcohol is driven for its positively reinforcing effects i.e. reward craving. However, after a period of prolonged episodes of intoxication a shift occurs. In this phase the use of alcohol becomes compulsive. The alcohol is now consumed to overcome the highly negative and aversive symptoms that is marked in the chronic state. This figure is reproduced with permission from Elsevier for printing in this thesis (14).

As mentioned, the clinical diagnosis of alcohol dependence includes tolerance and withdrawal. With repeated cycles of alcohol-intake and subsequent withdrawal, neurobiological and neurochemical responses to alcohol-intake are altered and increasing doses of alcohol are required to achieve the same effects. Abrupt cessation of alcohol-intake produces a rebound effect and is experienced as withdrawal symptoms.

Behaviorally, acute withdrawal from alcohol is characterized by tremor, increased heart rate, flush, sweating and high risk of epileptic seizures and delirium tremens. During protracted abstinence, affective symptoms include increased anxiety, negative mood and enhanced sensitivity to stress $(15,16)$. The individual feels highly motivated to offset these aversive symptoms by resuming alcohol consumption. Alcohol craving 
in the latter stage of AUD is thus driven primarily by negative reinforcement or behaviors that remove negative aversive affect and alcohol-intake becomes compulsive $(14,17,18)$. The specific neurobiological mechanisms behind the switch from positive to negative reinforcement mediating alcohol seeking and consumption are multiple and the exact mechanisms are largely unknown (18). Understanding these mechanisms is critical for the development of new and effective treatments for AUD.

\subsubsection{Risk factors for alcohol use disorder}

AUD arises from a combination of many personal, social and biological risk factors that directly and indirectly influence disease progression. A social context that permits alcohol use in everyday settings and approves of drinking to intoxication plays a critical role. It has been extensively reported that early and excessive alcohol use during adolescence increases the risk of developing AUD as an adult, with peer use being a strong predictor of adolescent alcohol use (19). Other risk factors include family history of AUD, lack of family support, low parental monitoring, childhood conduct disorders, mood disorders, low self-control, impulsivity and positive expectancies about alcohol use. Family history of AUD in particular predicts high risk of early onset and development of AUD due to a combination of genetic risk and behavioral effects of family members modeling heavy drinking (20).

In addition to environmental factors, genetics play a substantial role in development of AUD (21). A polymorphism of the enzyme alcohol dehydrogenase (ALDH) reduces risk of developing AUD (22). There are several other genetic variations that influence neurotransmitter responses to alcohol and that individually and modestly increase risk of developing AUD. Each identified allele that contributes adds less than $1 \%$ of the genetic risk for AUD, making AUD a polygenic disorder and indicating that although AUD is heritable, this is likely due to additive effects of many genes that contribute minor risk rather than having a clearly predictive genotype (23).

\subsubsection{Anxiety and depression are closely associated with alcohol use disorder}

Specific symptoms of different anxiety disorders vary, but all include some form of excessive, irrational fear and some form of anticipation of negative events (24). Common depressive symptoms include persistent sadness, hopelessness, guilt, irritability, loss of interest in activities that were once pleasurable, sleep abnormalities and suicidal thoughts. The severity, frequency and duration of these symptoms vary between individuals. There is high comorbidity between anxiety disorders, major depression, alcohol and other substance-use disorders and personality disorders (25).

Interestingly, anxiety disorder is among the third most common psychological disorder exceeded by depression and AUD (26). There is a strong relationship between anxiety and AUD. Several epidemiological studies have shown a remarkable co-morbidity 
between anxiety disorder and AUD $(26,27)$. Thus, we should focus more on exploring the potential factors that influences the relationship between anxiety disorder and AUD. Gender, situational context, drinking motives and alcohol expectancies are some of the interesting variables. Thus it is necessary to gain insight into which factors are the key players in the association between anxiety disorders and AUD and which might prove useful clinically in developing treatments for the patients with this common form of psychiatric co-morbidity (27).

Depression and AUD also frequently co-occur. Depression is a prevalent psychiatric disorder among drinker. Although the relationship between depression and alcohol use is well established, it is not known whether depression is an independent risk factor for developing alcohol dependence (28). Plenty of underlying mechanism defines the association between depression and alcohol-intake comorbidity along with the behavioral manifestations of depression $(29,30)$. Moreover depressive like symptoms weaken an individual's ability to self-control which influences the amount of alcohol-intake and risk to relapse (31).

\subsubsection{Neurobiological effects of alcohol}

Alcohol is one of the most socially accepted psychoactive drugs in modern society, despite the fact that the dangers of alcohol abuse and addiction are very well known. The neurobiological mechanisms underlying the transition from alcohol use to AUD remain unclear (32). Mechanisms are likely related to interactions between genetic susceptibility and contributing environmental factors; and to changes in the brain that occur with chronic exposure to alcohol (33).

Alcohol is considered a pharmacologically "dirty drug" meaning that it influences a broad range of neurotransmitter and neuropeptide systems in multiple brain areas (34). Moreover, different stages of development of alcohol addiction recruit different regions and sub-regions of the brain (Figure 2). Brain areas that are involved in regulating the rewarding effects of alcohol and other drugs of abuse include the ventral tegmental area (VTA), nucleus accumbens (NAc) and medial prefrontal cortex (mPFC) $(35,36)$. In the early stages of development of alcohol dependence, alcohol consumption is impulsive and driven by its pleasurable and positive reinforcing effects, i.e. reward. This leads to craving for alcohol reward in social contexts, followed by more frequent and heavier drinking. Over prolonged and repeated cycles of intoxication followed by abstinence and then relapse, a shift occurs. At this point, alcohol consumption becomes compulsive and drinking becomes necessary to relieve the negative and aversive affective symptoms that accompany withdrawal from alcohol. Negative affect and withdrawal involves brain regions such as the amygdala and bed nucleus of stria terminalis (BNST) (Figure 2). This process is associated with plasticity changes in central neuropeptide systems, suggesting recruitment of these systems during the transition to alcohol dependence $(37,38)$. 

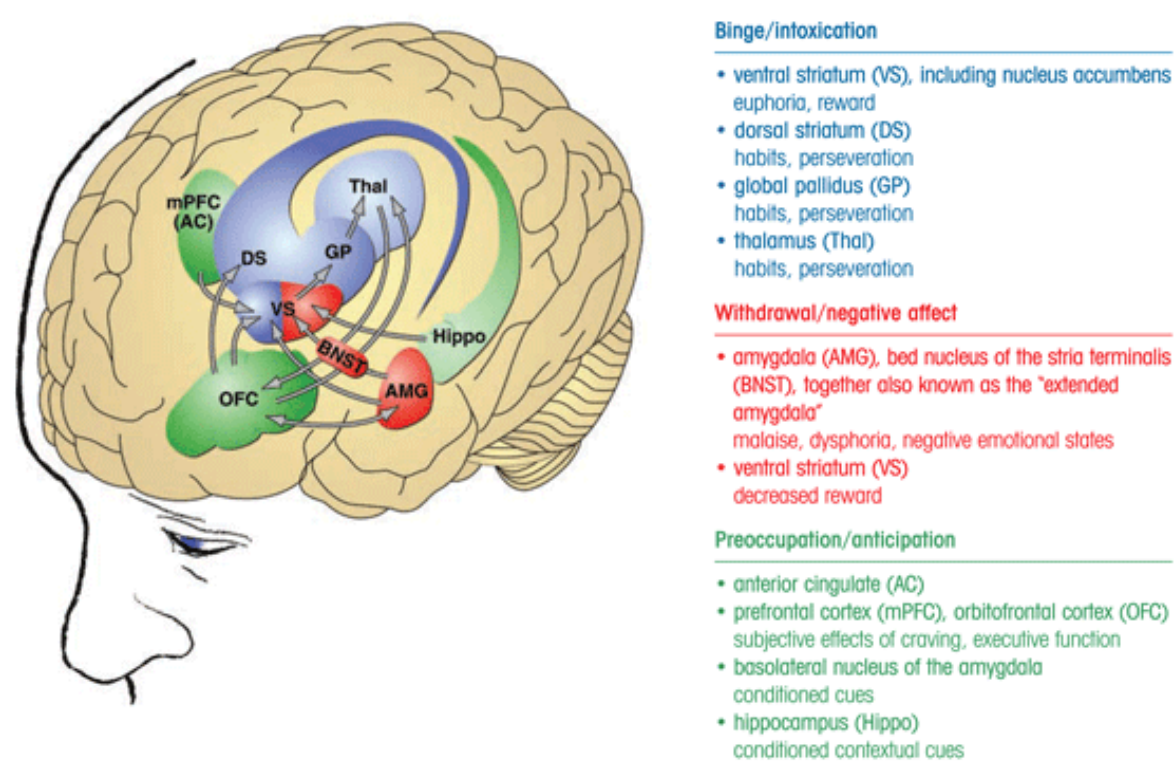

Figure 2. Neurocircuitry schematic illustrating the combination of neuroadaptations and key brain regions during the three proposed stages (binge/intoxication, withdrawal/negative affect and preoccupation/anticipation) of the alcohol addiction cycle that drive drug-seeking behavior. The striatum is activated during the binge/intoxication stage. During the withdrawal/negative affect stage dopamine systems are inhibited and the stress response is activated to further enhance the salience of drugs and drug-related stimuli in the context of an aversive dysphoric state. During the preoccupation/anticipation stage, contextual cues processed in the hippocampus and conditioned stimulus cues processed in the basolateral amygdala (BLA) converge with frontal cortex activity to drive drug seeking. Neurotransmission in other areas of the frontal cortex is inhibited causing deficits in executive function. This figure is reproduced with permission from Elsevier for printing in this thesis (38).

\subsubsection{Current treatment options for alcohol use disorder}

Given the prevalence and severity of alcohol use, abuse and dependence understanding how and where it acts in the brain is a key challenge in the development of novel treatments. The direct actions of alcohol within the central nervous system (CNS) are poorly understood (39) although mechanisms of action of alcohol via several classical neurotransmitters as well as several neuropeptides have been identified (39). The current number of available pharmacological treatments for AUD is limited to only three to five depending on the country (6).

Although AUD is a significant world health problem and socioeconomic burden only a few pharmacological interventions are available. Currently only three medications have been approved by Food and Drug Administration (FDA) in the United States for treatment of AUD. Those are: Disulfiram, Naltrexone and Acamprosate. The situation is similar in Sweden with those three medications plus nalmefene (trade name 
Selincro) being available to patients. All these treatment options available today have limited efficacy in preventing craving and relapse.

Disulfiram (trade name Antabuse) was the first treatment for alcohol addiction introduced in the market. The mechanism of action of disulfiram is to inhibit the enzyme ALDH thereby blocking the metabolism of alcohol and resulting in an accumulation of acetaldehyde in the body (40). This causes highly aversive symptoms such as sweating, flushing, nausea and vomiting if alcohol is consumed after taking the medication. These effects are potentially dangerous and can even be fatal. The accumulation of acetaldehyde can have toxic effects on multiple organs including the liver where it is mostly metabolized (41). Thus, it is very important that the patient is well informed about the actions and the possible adverse effects of this medication and preferably taken under medical supervision. The aim with disulfiram was to establish a negative association between alcohol use and aversive feelings to help the patient to refrain from drinking. However, it has been shown that disulfiram has no significant impact on withdrawal symptoms or the desire to consume alcohol. Compliance is low in the absence of medical supervision and randomized controlled trials show no difference from placebo in promoting abstinence (42).

Naltrexone (trade names Vivitrol and Revia) an opioid receptor antagonist that preferentially binds to $\mu$ and $\kappa$ receptors at the doses used clinically was approved by the FDA in 1984 for treatment of opiate dependence and AUD. The mechanism of action of is not clearly understood but it is thought that it modulates alcohol-induced dopamine transmission to reduce the rewarding effects of alcohol which has been the behavioral outcome of studies with social drinkers and patients with AUD $(43,44)$. Treatment outcomes are variable likely because this treatment is most effective in male individuals carrying a specific variant of the OPRM1 gene (45).

Acamprosate (trade name Campral) is the most recent medication to be approved for the treatment of AUD in the United States. It acts to reduce craving and promote abstinence (46). It is chemically similar to that of GABA and its primary mechanism of action appears to be reducing glutamatergic neurotransmission though this is complicated and fairly controversial. At high concentrations according to various studies acamprosate inhibits glutamate receptor activation, enhances NMDA receptor function, acts as a weak antagonist at the NMDA receptor, acts as a partial agonist at the polyamine site of the NMDA receptor and possibly inhibits the mGluR1 and mGluR5 receptors. However, no direct action of acamprosate at clinically-relevant concentrations has been discovered. Preclinical data has recently suggested that the mechanism of action of acamprosate at clinically relevant doses may be due to its function as a carrier of calcium into the nervous system (47). 


\subsection{Neurotransmitter systems in alcohol use disorder}

Alcohol does not have a single specific receptor or transporter site of action in the CNS. Upon ingestion, it affects the general homeostasis of the brain by modulating numerous neurotransmitter and receptor systems $(32,48,49)$. This makes it challenging to identify which specific factors are affected during the transition from controlled to compulsive alcohol drinking. Several neurotransmitter systems that have are recruited and implicated in regulation of the rewarding effects of alcohol include classical transmitters such as GABA, glutamate, dopamine and serotonin systems, as well as neuropeptide systems such as the opioids, etc (50-53) (Figure 3). The neuropeptide systems (nociceptin/orphanin FQ (NOP), neuropeptide Y (NPY) and melanin-concentrating hormone $(\mathrm{MCH})$ ) which are the focus of this thesis are presented separately in the next sub-chapter 1.4, 1.5 and 1.6.

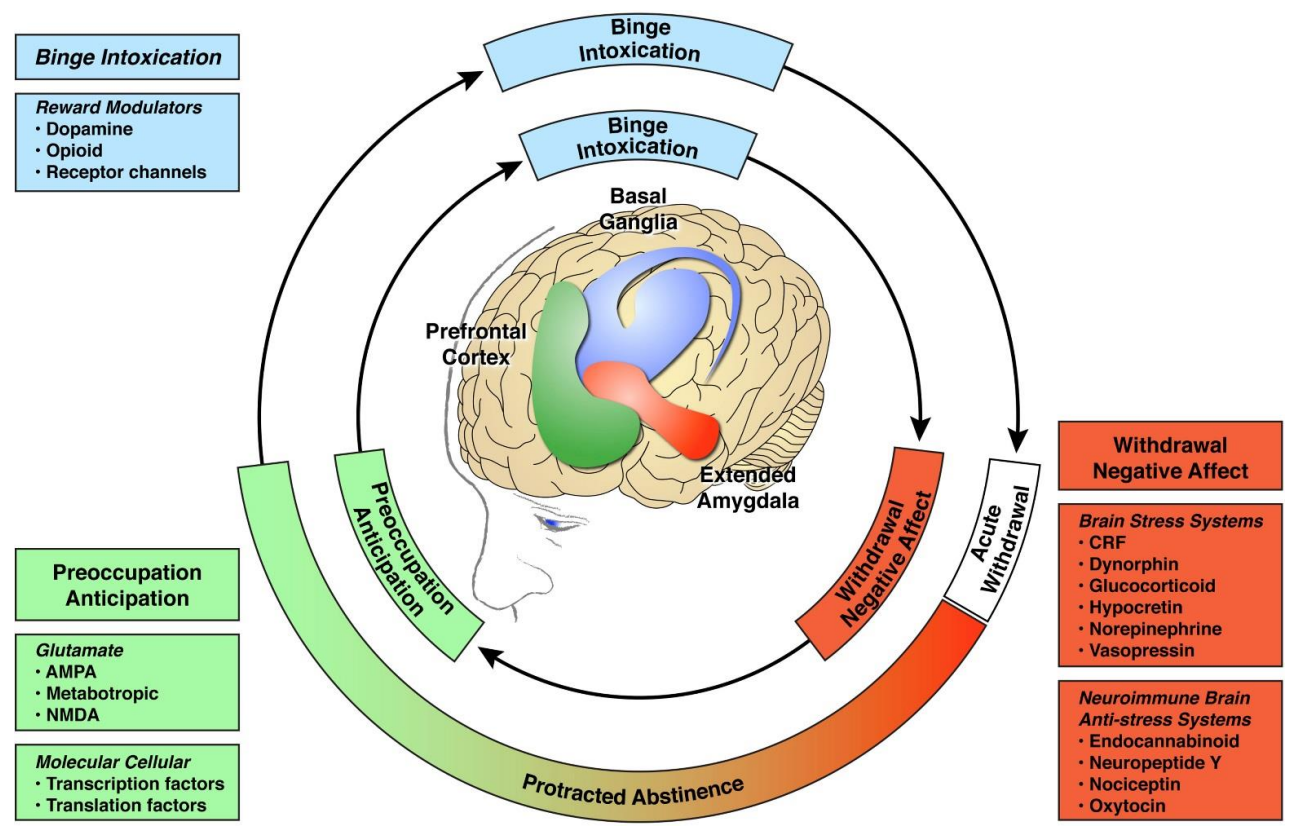

Figure 3. This pictorial presentation highlights the novel targets by focusing on the three key stages (binge/intoxication, withdrawal/negative affect and preoccupation/anticipation) of the AUD cycle with potential molecular targets. Mason et. al 2017 summaries and expands on the emerging and potential pharmacotherapies for AUD with corresponding clinical states (54). This figure is reproduced with permission from Elsevier for printing in this thesis. 


\subsubsection{The GABAergic system in alcohol use disorder}

The depressant effects of alcohol result from its agonist activity at GABAA receptors the principal postsynaptic receptors for the inhibitory neurotransmitter GABA. Other agonists at these receptors include barbiturates, anesthetics and benzodiazepines. GABA release opens the ion channel allowing chloride ions $\left(\mathrm{Cl}^{-}\right)$to enter the neuron resulting in hyperpolarization of the membrane which leads to delay and/or inhibition of action potentials (55).

Chronic and repeated exposure to alcohol results in reduced sensitivity of $\mathrm{GABA}_{A}$ receptors to alcohol and to GABA (56). This results in an increased tolerance to the effects of alcohol such that more alcohol is needed in order to achieve the desired depressant and intoxicating effects. In order to achieve homeostasis the CNS recruits glutamate resulting in symptoms such as tremors and anxiety during withdrawal phase. Over time continued exposure to alcohol promotes further CNS hyperexcitability as a result of hypersensitivity of glutamate receptors $(57,58)$.

$\mathrm{GABA}_{A}$ receptors also regulate dopamine levels in the mesolimbic dopaminergic system. Injecting $\mathrm{GABA}_{\mathrm{A}}$ agonist muscimol directly into the VTA dose-dependently increases dopamine release from the VTA to the NAc (59). Long-term exposure to alcohol decreases $\mathrm{GABA}_{A}$ receptor expression in the VTA which also results in increased dopamine release (60).

\subsubsection{The glutamatergic system in alcohol use disorder}

Glutamate is the primary excitatory neurotransmitter and acts through the ligand gated ion channel (LGIC) receptors, N-methyl-D-aspartate (NMDA), kainic acid and $\alpha$ amino-3-hydroxy-5-methyl-4-isoxazolepropionic acid (AMPA) as well as through the G-protein coupled metabotropic glutamate receptors (mGluR1-8). These receptors are widely distributed throughout the brain with the highest density in the forebrain (61, 62). Glutamate can be neurotoxic at high concentrations and its synaptic concentration is therefore tightly controlled. Glutamate is rapidly removed after release into the synaptic by excitatory amino acid transporters EAAT1-5, EAAT1 and EAAT2 predominantly found in glial cells, are responsible for the majority of glutamate reuptake from synapses. EAAT3-5 are expressed in neurons throughout the brain (63). Glutamate is important for neuronal plasticity, learning, memory and has been implicated in the pathophysiology of anxiety disorders and AUD.

Results from studies using in-vivo microdialysis in rats show that repeated cycles of alcohol exposure and abstinence lead to elevated levels of extracellular glutamate (64). This finding is further supported by a recent study showing that mice with a targeted mutation of the clock gene Per2 had increased levels of glutamate in the ventral striatum driven by impaired function and expression of glutamate transporters 
and also had increased drinking in the two-bottle free choice alcohol drinking paradigm (65).

Glutamatergic signaling in the mPFC, amygdala and hippocampus is important in the regulation of alcohol reward with enhanced signaling in initial stages of the reinforcing effects of acute alcohol followed by reduced glutamatergic activity. Acute alcohol also markedly attenuates extracellular glutamate levels in the NAc. NMDA and mGluR5 are the glutamate receptor subtypes most directly involved in modulating the effects of alcohol. Glutamate is critically involved in the neuroplasticity that occurs as dependence develops and in the neuronal hyperexcitability as well as in craving that occur during abstinence $(66,67)$.

Treatment with acamprosate an approved treatment for AUD that proposedly acts at NMDA receptors was shown to stabilize the elevated glutamate levels, reduce alcohol consumption and promote abstinence (46). Therefore, it has been hypothesized that either downregulation or inhibition of glutamate transporters may mimic the hyperglutamatergic state observed after repeated cycles of alcohol exposure and that withdrawal could be due to downregulation or inhibition of glutamate transporters (14). It has been shown that glutamate transporters were upregulated in the PFC of patients with AUD (68).

It should be noted that calcium has been suggested to be the active moiety of acamprosate (69). Recently it has been reported that plasma calcium concentrations in association with severity of alcohol dependence and its interaction with regulating pathways and alcohol craving in alcohol-dependent patients (70).

In rodent models, acute stress produces robustly elevated glutamate release in the hippocampus and PFC (71). Patients with major depression also have elevated levels of glutamate possibly resulting from a broad loss of glial cells and reduced expression of EAAT1-2 in the frontal regions of brain (72-74).

\subsubsection{The dopaminergic system in alcohol use disorder}

The mesocorticolimbic dopaminergic system is altered in patients with AUD. This neurotransmitter system consisting of dopaminergic neurons projecting from the midbrain VTA to the cortical mPFC and the limbic system NAc is known as the "reward pathway or hub" and is a key factor in the development of AUD. Alcohol acts directly on VTA interneurons to stimulate dopamine release from dopaminergic neurons projecting from the VTA to the NAc. This process is critical in the mediation of drug seeking, positive reinforcement and reward learning. As previously mentioned, another effect of alcohol-intake is to enhance $\beta$-endorphin release and its activity at MOR in the VTA results in inhibition of the GABAergic interneurons that normally exert tonic inhibition of the dopaminergic neurons in the VTA. The net effect is enhanced dopamine release into the NAc $(36,75,76)$ (Figure 4). 
The neuronal population of the NAc consists of $90-95 \%$ GABAergic projection neurons called medium spiny neurons that contain dopamine D1 or D2 receptors (77). The neurons expressing D1R primarily contain the neuropeptides substance $P$ and dynorphin (DYN) and project back to the VTA or to the substantia nigra defined as the direct pathway (78). The neurons expressing D2R contain the neuropeptides neurotensin and enkephalin and project to limbic regions including the ventral pallidum defined as the indirect pathway $(79,80)$.

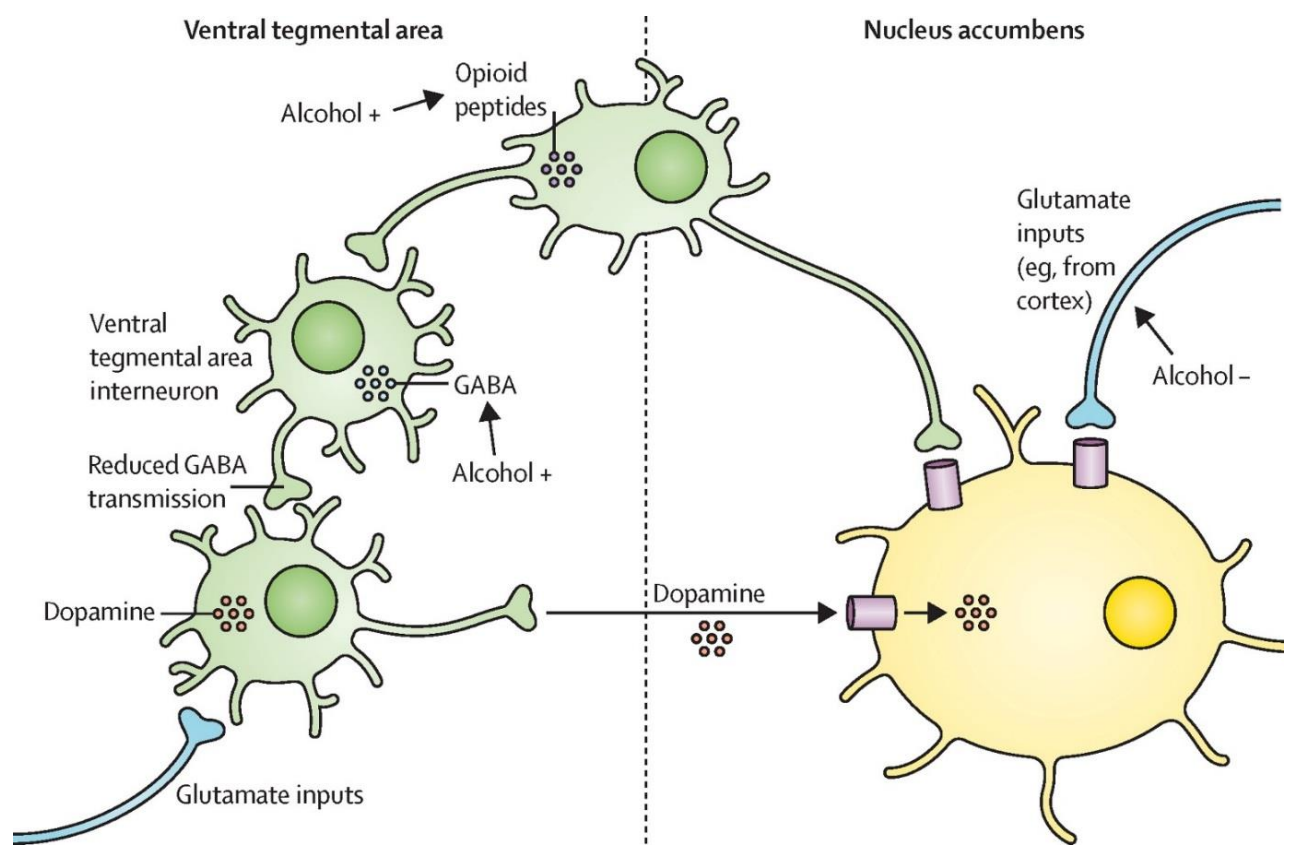

Figure 4. The effects of alcohol on $\mathrm{GABA}_{\mathrm{A}}$, glutamate and dopamine in the mesolimbic reward pathway. Alcohol disinhibits GABAergic transmission in the VTA resulting increased dopamine release into the NAc. Consequently, alcohol inhibits glutamate release of in the VTA and NAc. It should be noted that several other neuropeptides may also affect regulation of alcohol reward in this pathway. This figure is reproduced with permission from Elsevier for printing in this thesis (1).

\subsubsection{The opioid system in alcohol use disorder}

Significant experimental evidence implicates the endogenous opioid system with the processes of reward and reinforcement. Actually many behaviors associated with reward and reinforcement for example feeding behavior are controlled by distinct components of the endogenous opioid system located in relevant brain regions (81). Opioids act by stimulating specific membrane receptors of which there are three major types. Those are: $\mu$ (mu), $\delta$ (delta) and $k$ (kappa). 
Alcohol stimulates the release of endogenous $\beta$-endorphin the primary ligand for muopioid receptors (MOR) resulting in alcohol's euphoric effects. This process affects the synthesis of endogenous opioid peptides and the binding properties of their receptors. Blocking activity at mu-opioid receptors with for example compounds with opioid receptor antagonist properties such as naltrexone reduces craving for alcohol and has been useful in the treatment of AUD for some patients (82).

Rats made dependent on alcohol via chronic exposure to alcohol vapor increased their operant responding for alcohol and also displayed negative affect and increased DYN immunoreactivity and $\mathrm{k}$-opioid receptor (KOR) signaling in the central nucleus of the amygdala (CeA) (83). DYN and KOR have been implicated in various psychiatric disorders including anxiety, depression and drug addiction. Rodent strains with genetically predisposition to consume more alcohol have reduced DYN/KOR tone in the brain reward circuitry. Both acute and chronic exposure to alcohol upregulate the DYN/KOR system (84-86). KOR antagonists have been shown to reduce the negative affect associated with stress and with repeated cycles of alcohol-intake and withdrawal (87). These antagonists are effective in reducing alcohol-intake but more under conditions of high levels of alcohol-intake in combination with exposure to stress than during the initial phase of AUD development. These results support a significant role for the DYN/KOR system in the development of alcohol dependence and increased alcohol consumption induced by stress (88). Thus targeting KORs in the development of novel therapeutics for AUD and related affective disorders could be promising.

Studies examining the $\delta$-opioid receptor (DOR) in alcohol-intake have yielded inconsistent results using pharmacological approach on alcohol consumption. DOR receptor expression densities were evaluated in brain regions of rodents that differed in alcohol avidities clarifying the role of DOR in alcohol-intake $(89,90)$. DOR knockout mice drink more alcohol and influences alcohol-intake partly through an effect of it on anxiety (91). Interestingly a long term exposure to alcohol downregulates MOR in NAc and striatum but has no effect on DOR (92). These data suggest that DOR may influence and plays a role in alcohol-intake and related behaviors making it a possible attractive target for the treatment of AUD.

\subsubsection{The serotonergic system in alcohol use disorder}

Serotonin $(5 \mathrm{HT})$ also regulates dopaminergic activity. Alcohol-preferring $(\mathrm{P})$ rats selectively bred for high two-bottle free choice alcohol drinking have fewer serotoninergic neurons, higher levels of endogenous opioids and more GABAergic neurons in the limbic system than alcohol-non-preferring (NP) rats selectively bred for low two-bottle free choice alcohol drinking (93). This results in reduced dopamine and a lower density of postsynaptic dopamine D2 receptors in $\mathrm{P}$ rats. Drugs that enhance $5 \mathrm{HT}$ release or dopamine release onto D2 receptors decrease alcohol consumption and dopamine D2 receptor antagonists increase alcohol consumption. Dopamine release is higher in $\mathrm{P}$ rats than in NP rats after alcohol consumption which suggests 
that $\mathrm{P}$ rats are more sensitive to the effects of alcohol. These findings are similar to results from human studies. Levels of $5 \mathrm{HT}$ metabolites in the cerebrospinal fluid (CSF) are lower in patients with AUD than in matched controls. Patients with AUD have reduced craving for alcohol, reduced stress and reduced probability to relapse when treated with serotonin and dopamine precursors (94).

\subsubsection{Stress systems in alcohol use disorder}

Endogenous stress systems are recruited during the late phase of AUD which is associated in mediating aversive feeling and negative emotional states. This negative affective state is remarkable at this late phase of addictive process characterized by elevated anxiety, dampened mood and hypersensitivity to stress response. Thus the craving for alcohol in this stage is compulsive which is mainly driven by negative reinforcement and insensitivity to negative consequences. The hypothalamus is not only involved in processing reward-related behaviors but also plays a key role in stress regulation as part of the hypothalamic-pituitary-adrenal (HPA) axis which is activated by both psychological and physiological stressors (95). Thus screening drugs that target the withdrawal and negative affect stage in AUD is important.

Corticotropin-releasing hormone $(\mathrm{CRH})$ is the primary neuropeptide involved in stress regulation and is an important component of the brain stress system. It is synthesized in the paraventricular nucleus (PVN) of the hypothalamus which is central in the regulation of the endocrine stress response system and contains a high density of cell bodies that produces $\mathrm{CRH}(96,97)$. Adrenocorticotropic hormone (ACTH) is released upon activation of the anterior pituitary gland by $\mathrm{CRH}$. ACTH is secreted into the blood and travels to the adrenal cortex, resulting in glucocorticoid release. The primary glucocorticoid in rodents is corticosterone and in humans is cortisol. CRH containing cell bodies project to extrahypothalamic regions including CeA and BNST that are involved in mediation of both fear and anxiety $(98,99)$. Also growing bodies of evidence and data makes $\mathrm{CRH}$ an attractive and novel target for the treatment of AUD.

On the other hand, DYN which binds to the KOR is another stress-related neuropeptide that is involved in regulation of the negative reinforcing effects of alcohol. The DYN/KOR system undergoes neuroadaptations following chronic exposure to alcohol and increased signaling during withdrawal period promotes the negative affect that drives subsequent excessive alcohol-intake (88). 


\subsection{Central neuropeptides in alcohol use disorder}

As previously mentioned, numerous neurotransmitter and neuropeptide systems are involved and recruited during the progression of AUD from intake due to reward to heavy consumption; and the anxiety associated with withdrawal and subsequent relapse. Several neuropeptides have been implicated in control of the reward and anxiety aspects of the pathophysiology and neuronal mechanisms of AUD.

The work in this thesis focuses on three of these neuropeptide systems: NOP, NPY and $\mathrm{MCH}$ that play roles in the neuroadaptation that occurs during the progression of the disease. These neuropeptides have been studied as possible therapeutic targets to alleviate and abolish the harmful effects of alcohol (100-102).

\subsubsection{G protein-coupled receptors}

Neuropeptides exert their functions within the CNS via G protein-coupled receptors (GPCRs) also known as 7-transmembrane domain receptors. GPCRSs are the most common target of drugs prescribed to treat CNS, cardiovascular, pulmonary and gastrointestinal diseases (103). GPCRs have a structure that passes through the cell membrane seven times creating a cavity in the membrane. The GPCRs detect molecules (ligands) on the outside of the cell and then activate internal pathways via coupling to G-proteins and for some also to beta-arrestin resulting in cellular responses (104).

The two principal signal transduction pathways following activation of GPCRs are primarily the cyclic adenosine monophosphate (cAMP) and the phosphatidylinositol signaling pathways. Binding of a ligand to a GPCR results in a conformational change in the GPCR that enables it to act as a guanine nucleotide exchange factor (GEF). GEFs are proteins or protein domains that activate monomeric guanosine triphosphatases (GTPases) by directly stimulating the release of GDP to allow binding of GTP. Thus, GPCR activates an associated G-protein by exchanging its bound GDP for GTP. The a subunit of the G-protein binds GTP and can then dissociate from the $\beta$ and $\gamma$ subunits to further activate or inhibit intracellular signaling proteins or target functional proteins directly depending on the a subunit type. This process is called signal transduction. A chemical or physical signal is transmitted through a cell via a series of molecular events most commonly of proteins which ultimately results in some response or effect $(105,106)$.

Beta-arrestin 2 a multifunctional key component of the GPCR complex is essential for $\mu$-opioid and dopamine D2 receptor signaling both of which are involved in mediating the rewarding effects of alcohol. Beta-arrestin can mediate receptor signaling independent of G-proteins and also mediates the cAMP cascade via a scaffolding complex with kinases and/or phosphatases $(107,108)$. Beta-arrestin 2 deficient mice have enhanced sensitivity to drug effects due to impaired desensitization of GPCRs 
which normally undergo desensitization via phosphorylation and subsequent binding of beta-arrestin to further prevent GPCR coupling $(109,110)$.

Beta-arrestin 2 knockout mice have sensitized dopamine release and reward response to a low dose of alcohol $(1 \mathrm{~g} / \mathrm{kg})$ and also drink more in the two-bottle free choice preference drinking paradigm. These mice also have enhanced morphine analgesia and reward response to morphine but a normal reward response to cocaine. These results suggest that beta-arrestin 2 normally functions as a positive regulator for alcohol- and opiate-mediated behavior (111-115).

\subsubsection{Biased Agonism}

GPCR targeted drug discovery is no longer limited to seeking agonists or antagonists to stimulate or block cellular responses that are associated with a particular receptor. GPCRs are now known to support a broad diversity of pharmacological profiles due to biased or functional selectivity. A ligand can stabilize subsets of receptor conformations to produce novel pharmacological profiles a concept known as ligand bias. Biased ligands can potentially offer safer, better tolerated and more efficacious drugs and several are currently in clinical development now. Biased ligands targeting GPCRs are thus promising for improved therapeutic profiles (116). 


\subsection{Nociceptin/Orphanin FQ}

Attempts to clone novel opioid receptor types and subtypes in 1994 led scientists from several laboratories to isolate cDNA encoding a protein with a primary structure analogous to those of opioid receptors that was named opioid receptor like-1 (ORL1) (117). ORL1 is a GPCR with high structural homology to the classical opioid receptors. However, when transfected into mammalian cells ORL1 does not show the activation by or binding affinity for classical opiates that the other opioid receptors does. ORL1 can be activated by a high concentration of opioid agonist etorphine and inhibited by a high concentration of opioid antagonist naloxone (117) but due to the lack of a good high affinity ligand there was no binding assay available to characterize this receptor. Thus it remained an "orphan" until late 1995 when two research groups independently reported isolation of the endogenous ligand which one group called nociceptin (118) and the other called orphanin FQ (119) thereafter referred to as nociceptin/orphanin FQ (NOP) (120). This 17 amino acid new neuropeptide was named nociceptin due to its enhancement of the nociceptive response in the hot plate test when administered intracerebroventricularly (ICV) to mice (118) and the name orphanin FQ denotes the ligand's first and last amino acids phenylalanine (F) and glutamine (Q) (121).

NOP a heptadecapeptide with amino acid sequence Phe-Gly-Gly-Phe-Thr-Gly-AlaArg-Lys-Ser-Ala-Arg-Lys-Leu-Ala-Asn-Gln is structurally related to the opioid peptide dynorphin- A (118). Despite its structural similarity to other opioid peptides NOP does not bind to classical opioid receptors nor do other opioid peptides bind to ORL1 receptor (121). Similarly to the other opioid receptors, activation of ORL1 by NOP triggers intracellular signaling events such as negative coupling with adenylyl cyclase activation of inward rectifying potassium ion channels and inhibition of calcium current $(118,119,121)$. These cellular signaling responses to NOP are relatively insensitive to opioid receptor antagonism with naloxone (122). Structural analysis suggests that separate mutations led to a coordinated distinct pharmacological separation of the NOP system from the opioid system. Brain mapping studies have shown that the neuroanatomical distribution of NOP and its receptor differs significantly from that of the other opioid peptides and receptors (123-125).

\subsubsection{Tissue distribution of the nociceptin/orphanin FQ receptor}

The focus of research on ORL1 was initially pain and nociception but this receptor is also expressed in brain areas involved in reward processing and stress response. ORL1 mRNA has been detected in cortical and corticolimbic areas including the amygdala, hippocampus, habenula and septum, the ventromedial and paraventricular nuclei of the hypothalamus, the locus coeruleus, parabrachial nucleus, periaqueductal gray and dorsal raphe nucleus in the brain stem and the dorsal and ventral horns of the spinal cord (126). Immunohistochemical mapping of the ORL1 protein in the rat CNS revealed similar distributions as those obtained from in-situ hybridization studies, indicating that ORL1 is predominantly expressed in local-circuit interneurons (124). The distribution of ORL1 mRNA and protein suggests that it could be involved in 
regulation of reward-related behaviors and in psychiatric disorders such as anxiety and depression. NOP may also play a role in regulation of drug intake and related behaviors.

\subsubsection{Functional role of nociceptin/orphanin FQ}

As described above in the text that NOP and its receptor are found in brain areas involved in reward processes and in regulation of anxiety- and stress-related behaviors. NOP can be considered an endogenous antagonist of dopamine transport in that it affects locomotion and other motor behaviors partly by inhibiting the dopamine transporter directly and partly by inhibiting GABA transporter to indirectly reduce dopamine transmission (127). In addition to modulating nociception and locomotion, NOP is also involved in food-intake, learning and memory and control of neurotransmitter release at central and peripheral sites (128). In addition agonism at the NOP receptor together with antagonism at classical opioid receptors may help to decrease drug intake and rewarding effects experience in patients who do not respond to other treatments (129).

NOP acts as a potent anti-analgesic when acting centrally in the forebrain efficiently counteracting the effects of pain medications (130). ORL1 is found in both central and peripheral nervous tissue where it modulates pain perception in the opposite direction of the classical opioid receptors. Unlike morphine and other opioids with pain-relieving properties administration of NOP directly into the brain causes increased sensations of pain or hyperalgesia. It also counteracts analgesia, thus acting as an anti-opioid, perhaps useful as a treatment for opiate overdose. Blocking ORL1 on the other hand, increases pain threshold and reduces the development of tolerance to analgesic opioids (131). As such NOP may have lower addiction potential than other commonly prescribed pain medications. The anti-analgesic function of NOP is mediated by inhibition of activity in the periaqueductal gray which controls pain modulation directed from the CNS (130-132). NOP has potential as a method to reduce morphine dose and decrease the development of tolerance and dependence in pain patients (133).

Despite high structural similarity to dynorphin-A $(119,134)$ NOP lacks the N-terminal tyrosine necessary for affinity at the classical opioid receptors $(121,135,136)$. Even at a nanomolar concentration range NOP selectively binds to ORL1 but not to other opioid receptors. Electrophysiology reveals that NOP inhibits the activity of $\beta$ endorphin containing neurons in the arcuate nucleus of the hypothalamus by activating inward potassium ion influx (137). This is likely to influence reward processing via interacting with the mesolimbic dopaminergic system given that these neurons projects to the VTA and NAc where reduced opioid release would affect behavioral response to a rewarding stimulus (138). 


\subsubsection{The role of nociceptin/orphanin FQ system in reward processing}

In addition to nociceptive effects the endogenous NOP system has also been implicated in a broad range of additional central and peripheral effects including drug addiction. For example ICV administration of NOP reduced voluntary alcohol-intake in the alcohol-preferring msP rat (139-141). In addition systemic (IP) administration of the NOP receptor agonist Ro64-6198 reduced anxiety (142) and alcohol selfadministration and prevented relapse-like behavior in an alcohol deprivation model in rats (143). It has also been reported that ICV administration of nociceptin reduces the rewarding effects of several drugs of abuse in the conditioned place preference (CPP) paradigm. On the other hand systemic administration of the NOP receptor antagonist $\mathrm{J}-113397$ enhanced the acquisition of cocaine-induced CPP and ICV administration of the peptide blocks it. NOP receptor antagonist UFP-101 enhanced the acquisition of methamphetamine-induced CPP in mice. Moreover, genetically modified mice lacking ORL1 are more susceptible to the rewarding effects of cocaine, methamphetamine and alcohol $(144,145)$. NOP given ICV has been shown to suppress dopamine release into the NAc in anesthetized rats an indication that it may have aversive properties (146). However, ICV administration of NOP is motivationally neutral in the CPP test in rats (147).

Data from clinical studies indicate that alcohol consumption increases during stressful life events and exposure to stress can trigger alcohol relapse in abstinent alcoholics. Central administration of NOP has an anxiolytic effect and knockout mice lacking the NOP gene have impaired adaptation to repeated stress exposure (148). Low doses of NOP reduce acute footshock-induced anxiety in rats (149). Chronic ICV injection of NOP significantly reduces two-bottle free choice alcohol-intake and preference in alcohol-induced CPP. Lastly, NOP inhibits cue- and stress-induced reinstatement of alcohol-seeking behavior in alcohol preferring rats $(150,151)$. Taken together, these results suggest ORL1 as a potential target for developing pharmaceutical interventions for AUD. Because NOP receptor activation may include differential activation of intracellular signaling pathways following ligand binding investigations into NOPbased pharmaceuticals should include tests for possible biased mechanisms.

\subsubsection{The nociceptin/orphanin FQ system in alcohol use disorder}

In animal models central administration of NOP has been shown to significantly decrease operant alcohol self-administration and reinstatement of alcohol seeking behavior suggesting a reduction in the experience of the rewarding properties of alcohol (140). Within the mesolimbic dopamine system over $80 \%$ of dopaminergic neurons in the VTA also contain ORL1 (152) which upon activation of ORL1 by ICV administration of NOP reduces the rewarding effects of alcohol by reducing dopamine release and by inhibiting the GABAergic pathway that is normally enhanced during alcohol reward and development of dependence $(153,154)$. 
It has also been demonstrated that the NOP agonist Ro64-6198 significantly blocks acquisition, expression and reinstatement of alcohol-induced CPP, self-administration and prevents relapse-like alcohol drinking $(143,155)$. The NOP antagonist J-113397 blocks the effects of the NOP receptor agonist Ro65-6570 on the acquisition of opiateand psychostimulant-induced conditioned place preference in rats. Taken together, these findings further support the involvement of ORL1 in processing of the rewarding effects of several drugs of abuse $(129,150)$.

\subsubsection{Buprenorphine in the rewarding effects of morphine and alcohol}

Buprenorphine- a mixed agonist/antagonist at the classical opioid receptors has been used as an alternative to methadone for treatment of heroin dependence (156). At low doses buprenorphine acts as an effective analgesic whereas the antinociceptive effect is diminished at higher doses. This bell-shaped dose-response curve suggests a unique and complex mechanism of action (157). Buprenorphine has been shown to activate ORL1 to produce an antinociceptive effect. Thus NOP can be indicated as an anti-opioid peptide for its ability to reverse morphine-induced analgesia (158). This anti-opioid activity can be extended to the motivational effects of morphine. ICV administration of NOP at doses as low as 500-2000 ng abolished CPP induced by 3 $\mathrm{mg} / \mathrm{kg}$ morphine, whereas NOP alone did not induce CPP. At the same doses, NOP also abolished alcohol-induced CPP $(139,147)$. NOP reduces morphine-induced dopamine release in rats which suggests a mechanism for its effects on drug reward. Rewarding stimuli increase $c$-fos expression in the NAc-shell and this effect is due to activation of dopaminergic neurons. NOP at a dose of $1000 \mathrm{ng}$ completely blocks morphine-induced $c$-fos expression in the NAc-shell (159). Buprenorphine has a biphasic effect on alcohol drinking such that it increases alcohol drinking via stimulation of classical opioid receptors at lower doses and suppresses alcohol drinking via activation of ORL1 at higher doses (160). This finding suggests that the ability of buprenorphine to act as an ORL1 agonist of NOP could be a useful strategy for treatment of AUD.

This thesis focuses on the effect of administration of a NOP receptor agonist on motivation to self-administer alcohol, relapse to alcohol seeking and two-bottle free choice drinking (paper I); and the agonist is further evaluated in rat models of high and escalated alcohol consumption (paper II). 


\subsection{Neuropeptide $Y$}

Neuropeptide Y (NPY) is a 36 amino acid peptide belonging to the family of pancreatic peptides and was first isolated by Tatemoto in 1982 (161). It is one of the most highly evolutionarily conserved neuroendocrine peptides and it is expressed throughout the CNS with high levels in the limbic areas of the brain (162). NPY plays critical roles in basic physiological processes such as stimulation of food and water-intake, pain modulation, sleep regulation, suppression of anxiety, inhibition of seizure activity and cognition (163-165). Central administration of NPY promotes feeding behavior, reduces cerebrocortical excitability, reduces anxiety and potentiates the sedative and hypnotic effects of certain drugs (166-169). NPY is overall an inhibitory peptide and regulates neuronal hyperexcitability by suppressing hippocampal glutamatergic transmission (170-172). Previous studies have revealed that NPY has a major role in the pathophysiology of mood disorders including anxiety and depression and this has been associated with downregulation of NPY in animal models and in human depressed patients $(173,174)$. NPY has been hypothesized to function as a stress buffering system in the CNS (175). NPY in the extended amygdala is recruited during the transition to alcohol dependence (176).

Four functional G protein-coupled NPY receptors $Y 1$, Y2, Y4 and Y5 have been discovered in mammals (177). Y4 is mainly expressed in peripheral tissue while the other three subtypes are expressed in the CNS and mediate the central effects of NPY. The distribution and function of $Y 1$ and $Y 5$ receptors overlap to a large extent. Y2 receptors act predominantly as presynaptic NPY autoreceptors and as such they regulate and modulate the release of endogenous NPY into the synaptic cleft (177).

\subsubsection{Neuropeptide Y-Y2 receptors}

As mentioned above, NPY exerts its effects via activity at heterogeneous GPCRs with distinct ligand affinity profiles (178). The $Y 1$ and $Y 2$ subtypes are the most abundantly expressed in the CNS. The NPY-Y1 receptor requires the intact NPY peptide for activation while the NPY-Y2 receptor can also be activated by C-terminal fragments of the peptide. NPY-Y1 and Y2 receptors are likely involved in NPY's modulation of stress, anxiety and depression. It regulates the uptake and release of endogenous NPY. NPY-Y2 receptors are highly expressed in the amygdala, hypothalamus and hippocampus $(179,180)$.

Pre-synaptically located NPY-Y2 receptors are coupled to $G_{o / i}$ proteins and mediate feedback inhibition of neurotransmitter release $(181,182)$. Previous studies have demonstrated reduced anxiety in NPY-Y2 receptor knockout mice compared with wild type mice $(183,184)$. Moreover, mice lacking NPY-Y2 receptors were shown to have an improved ability to cope with stressful situations. Deletion of this receptor resulted in increased NPY and GABA release and decreased glutamate release in brain areas involved in processing of anxiety and stress (184). Activation of NPY-Y2 receptors also enhances anxiety in the open-field and social interaction tests in rats $(185,186)$. 
NPY-Y2 receptors act in part serve to negatively regulate NPY transmission and release in the brain. However, the anxiolytic effects of NPY are mediated by activation of NPY-Y1 receptors. An alternative means of activating NPY-Y1 receptors may be via antagonism at the presynaptic NPY-Y2 that may lead to potentiation of NPY transmission post synaptically $(187,188)$. Antagonism of NPY-Y2 receptors would thus be expected to increase NPY levels in the CNS and may prove useful as a candidate target for treatment of anxiety, depression and AUD (188-190).

\subsubsection{Neuropeptide $Y$ in the modulation of food and alcohol- intake}

Central administration of NPY reduces alcohol-intake in the alcohol-preferring $\mathrm{P}$ rat and suppresses alcohol-intake in rats regardless of history of alcohol exposure. Thus NPY is effective at reducing alcohol-intake even in models of high preference and dependence $(191,192)$. The NPY-Y2 antagonist BIIE0246 suppressed operant responding for alcohol in rats both naïve and with a history of dependence induced by long-term intermittent alcohol vapor exposure. However, the suppression in vaporexposed animals was seen at a dose that did not affect behavior in naïve animals, indicating that the NPY system is sensitized following a history of dependence. BIIE0246 did not affect self-administration of a non-alcohol reward, showing specificity of the effect for alcohol $(189,190)$. Another NPY-Y2 antagonist JNJ-31020028 transiently suppressed alcohol-preference but did not affect the overall intake. It did however, reverse the anxiogenic effects of alcohol withdrawal-induced anxiety measured on the elevated plus-maze which supports the anxiolytic properties of NPYY2 antagonism (188).

A direct correlation between NPY signaling and regulation of alcohol consumption was found in a study where mice with transgenic overexpression of NPY consumed less alcohol while mice with a complete elimination of the NPY gene had increased alcohol consumption (193). Moreover, differences in NPY expression in rat strains selected for high and low alcohol-preference were demonstrated (194). Post-mortem brain tissue from alcoholics has significantly lower NPY expression than that of healthy controls. Low expression of NPY in the amygdala promotes high levels of alcohol drinking while high levels of NPY in the hippocampus may play a protective role against excessive alcohol consumption (195). The hippocampal NPY system is downregulated during alcohol withdrawal and these neuroadaptive changes could play a role in mediating withdrawal hyperexcitability (196). ICV administration of NPY significantly reduces withdrawal symptoms in rats.

Allelic variation in the NPY gene contributes to the heritability of alcoholism. In humans there is a correlation between alcohol consumption and the Leu(7) to Pro(7) polymorphism of the NPY gene (197) such that the polymorphism is associated with increased alcohol consumption. In another study, a novel polymorphism at position 602 in the $5^{\prime}$ region of the NPY gene was identified as being significantly associated with AUD (198). 


\subsubsection{Neuropeptide $Y$ in anxiety}

Anxiolytic and sedative effects of exogenous NPY were the main initial findings of animal experiments. Acute administration of NPY reduces anxiety in a variety of animal models including the elevated plus-maze, Vogel conflict test, fear-potentiated startle, fear conditioning and the social-interaction test $(166,169,177,199)$. NPY deficient mice exhibit increased anxiety in the same models (200). NPY is expressed and released following stress and this results in reduction of stress-induced behaviors (201). This clearly suggests that NPY could be recruited to reduce the effects of prolonged stress. In agreement with this hypothesis transgenic mice overexpressing NPY in the hippocampus suppress anxiogenic responses to stress (167). The mechanism for this is unclear but it could be related to modulation of other systems such as inhibition of glutamate release (202), suppression of CRH release (203) and potentiation of GABA-mediated neurotransmission (204).

The anxiolytic effects of NPY are mediated by Y1 receptors (168). Activation of Y1 and $Y 5$ receptors in the amygdala produces an anxiolytic effect in rodents (186). Mice lacking $Y 1$ receptors exhibit anxious behavior in some tests and depending on the circadian cycle (205). Expression of NPY is differentially regulated by acute and chronic stress as revealed by studying changes in NPY expression in brain regions mediating stress responses (175). Upregulation of NPY receptors may help the individual adapt to anxiety and stress such that NPY acts as a buffer for the behavioral effects of stress-promoting hormones such as $\mathrm{CRH}$.

\subsubsection{Neuropeptide $\mathrm{Y}$ in depression}

In a rodent model of depression, animals exhibited a significantly reduced baseline level of NPY in the hippocampus compared with controls (206) and this decreased hippocampal NPY Y1 receptor expression was correlated with behavioral changes associated with depression. The phenotype was rescued by electroconvulsive therapy (ECT) that resulted in potentiated NPY levels (207). This result is in agreement with pharmacological studies demonstrating that antagonism of the $Y 1$ receptor blocks the antidepressant effects of $Y 1$ agonist (208). NPY Y1 receptors have been also suggested to modulate hippocampal neurogenesis (209). Overall, these data support involvement of the NPY-Y1 receptor in promoting antidepressant effects.

Human patients with major depression have decreased levels of NPY in the CSF (174) that increases after chronic treatment with antidepressants (210) and ECT (211). A haplotype block of the prepro-NPY gene carrying a functional -399T- $>C$ substitution is highly associated with late-onset AUD and the clinical subtype of AUD that is characterized by high trait of anxiety (212). This gene variant also represents a vulnerability factor for depression. Lastly, the functional importance of the NPY system and its genetic variation has been associated with stress responses in humans in fMRI studies (213). 
This thesis focuses on the role of a NPY-Y2 antagonist in anxiety, two-bottle free choice drinking, operant self-administer and motivation to obtain alcohol and relapse to alcohol seeking behavior in a rat model (paper III). 


\subsection{Melanin-concentrating hormone}

$\mathrm{MCH}$ initially named melanin-concentrating hormone $(\mathrm{MCH})$ was first discovered in 1983 in the pituitary gland of the fish chum salmon where it was shown to regulate ability to change skin color in response to predatory threat (214). Though the function of $\mathrm{MCH}$ has evolved in higher species to instead be involved in the regulation of energy homeostasis, its 19 amino acid cyclic peptide (215) structure is highly conserved in rats and humans (216).

The human $\mathrm{MCH}$ gene is located on chromosome 12 and consists of three exons and two introns. Three peptides, neuropeptide glycine glutamic acid (NGE), neuropeptide glutamic acid isoleucinamide (NEI) and $\mathrm{MCH}$ are encoded in the second and third exons. The physiological functions of NGE and NEI are still unknown. The gene that encodes for $\mathrm{MCH}$ generates the precursor pro- $\mathrm{MCH}$ which generates three peptides through differential processing that is dependent on the tissue in which it is expressed (217).

$\mathrm{MCH}$ cell bodies are located in the lateral hypothalamus ( $\mathrm{LH})$ and the zona inserta brain areas known to be involved in food-intake (218). $\mathrm{MCH}$ neurons project to the limbic system among other brain regions. Primates express two receptor subtypes $\mathrm{MCH} 1-\mathrm{R}$ and $\mathrm{MCH} 2-\mathrm{R}$ while rodents only express $\mathrm{MCH} 1-\mathrm{R}$ (219).

\subsubsection{Intracellular signaling pathways of $\mathrm{MCH}$ receptors}

The MCH peptide was discovered as the endogenous ligand for the MCH1-R in 1999. $\mathrm{MCH} 1-\mathrm{R}$ is highly expressed in the brain in both rodents and in humans and is also expressed in cardiac, hepatic and fat tissue. $\mathrm{MCH} 1-\mathrm{R}$ expression in the brain is highest in the limbic system and particularly in the NAc and striatum $(220,221) . \mathrm{MCH} 1-\mathrm{R}$ is a GPCR and signals through $G_{i}, G_{0}$ and $G_{q}$ pathways. The most preferred pathway is through $G_{i}$ which directly inhibits cyclic adenosine monophosphate leading to activation of protein kinase A signaling pathway which then leads to induction of mitogen-activated protein kinase (MAPK) signaling cascades. Signaling through G。 activates the MAPK pathway through a different mechanism recruiting protein kinase C. Signaling through $\mathrm{G}_{q}$ increases calcium levels and excitability of the cell resulting in mobilization of the endoplasmic reticulum and subsequent enhancement of vesicle packaging of and exocytosis $(222,223)$.

After the discovery of $\mathrm{MCH} 1-\mathrm{R}$ another similar receptor with $38 \%$ sequence homology was identified and named the $\mathrm{MCH} 2$ receptor. Activation of $\mathrm{MCH} 2-\mathrm{R}$ leads to the stimulation of the $\mathrm{G}_{q}$ signaling pathway. The distribution of $\mathrm{MCH} 2-\mathrm{R}$ is similar to that of $\mathrm{MCH} 1-\mathrm{R}$ and it likely shares similar functions. $\mathrm{MCH}$ is the only known ligand for either receptor subtype. The pro-MCH-derived peptides $\mathrm{NGI}$ and $\mathrm{NEI}$ do not bind to $\mathrm{MCH}$ receptors (224). 


\subsection{2. $\mathrm{MCH}$ in modulation of food-intake and energy homeostasis}

For all living organisms regulation of energy homeostasis is critical for survival. Signaling between the brain and multiple peripheral organs modulates this process. The hormones insulin, leptin and ghrelin act on neurons in the arcuate nucleus of the hypothalamus to regulate of food-intake and energy expenditure (225). Signaling in these neurons is also regulated by orexigenic neuropeptide NPY, anorectic neuropeptide proopiomelanocortin (POMC) and synapses onto secondary $\mathrm{MCH}$ neurons in the LH (226). Inhibition of POMC and activation of NPY neurons triggers hunger. This process results in stimulation of $\mathrm{MCH}$ that results in food-intake. Satiety has the opposite effect on POMC and NPY which inhibits $\mathrm{MCH}$ secretion and in turn food-intake.

Preclinical rodent studies have shown that acute or chronic administration of $\mathrm{MCH}$ increases food-intake and body weight leading to an obesity phenotype (227). In line with this genetic deletion of the pro-MCH gene (Pmch) results in reduced food-intake and body weight leading to a lean phenotype (227) while overexpression of this gene has the opposite result (228). Similarly, antagonism of MCH1-R reduced food-intake and body weight in normal rats and in a rat model of obesity (229). These results suggest that targeting the $\mathrm{MCH}$ receptor could be a useful strategy in treating obesity. Local injections of $\mathrm{MCH}$ into several different nuclei of the hypothalamus result increase in food-intake (230). Similarly, local injections of MCH into the NAc increased food-intake and this effect can be blocked with an MCH1-R antagonist (231). These results illustrate that there is overlapping circuitry regulating food reward and the rewarding properties of drugs of abuse.

\subsubsection{Shared mechanism in the regulation of food and drug reward}

Natural rewards such as palatable food and drugs of abuse activate the same mesolimbic dopaminergic reward pathway. Consumption of palatable food triggers the release of endogenous opioids resulting in activation of opioid receptors in the VTA. This leads to dopamine release into the NAc. The same pathway is activated upon alcohol consumption (232-234). Consumption of sugar a component of many palatable foods can result in a phenotype similar to that observed with intake of drugs of abuse in preclinical rodent models. Rats trained to self-administer foods or drinks containing sugar escalate their consumption over time in a way that reflects the behavioral pathology of addiction $(235,236)$. Chronic IA to sugar results in compensatory downregulation of opioid and dopamine receptors similarly to what is observed with chronic IA to alcohol and these rats display a varying degree of withdrawal symptoms when the sugar is removed $(233,237)$.

In humans, drug dependence is often comorbid with binge eating disorder suggesting that the underlying mechanisms regulating these pathophysiological states are mediated by the overlapping neural pathways (238). It is important to note that the 
NAc which regulates reward and motivation for drugs of abuse also receives innervation from the hypothalamus (239) a brain area that contains several orexigenic and anorectic peptides involved in the regulation of food-intake and energy homeostasis $(240,241)$. Innervation and input from the hypothalamus to NAc is an important pathway for the regulation of food consumption and reward (242-244). Because alcohol is an addictive drug with caloric value it could be important to target hypothalamic neuropeptides as a potential new therapeutic intervention for AUD.

\subsection{4. $\quad \mathrm{MCH}$ in modulation of stress and anxiety}

$\mathrm{MCH}$ modulates activation of the HPA axis $(96,218)$. ICV administration of $\mathrm{MCH}$ increases corticosterone levels in rats and this effect can be reversed by pretreatment with a $\mathrm{CRH}$ antibody (245). Local injection of $\mathrm{MCH}$ into the paraventricular nucleus of the hypothalamus (PVN) elevates ACTH and plasma corticosterone in rats. These results suggest that $\mathrm{MCH}$ can activate the HPA via $\mathrm{CRH}$ system (246). Abnormalities in HPA axis function have been associated with depression, chronic stress and AUD (247).

The MCH1-R also interacts with stress modulating circuitry in limbic regions such as the hippocampus, amygdala and PFC. Central administration of $\mathrm{MCH}$ has anxiolytic effects in the elevated plus-maze and the Vogel punished drinking test $(248,249)$. In contrast, an $\mathrm{MCH} 1-\mathrm{R}$ antagonist has been shown to reduce anxiety in the social interaction test and depression in the forced swim test (FST) (250). Local injection of an $\mathrm{MCH} 1-\mathrm{R}$ antagonist into the $\mathrm{NAc}$ reduces whereas local injection of $\mathrm{MCH}$ into the NAc enhances depression in the FST (231). Several small molecule MCH1-R antagonists have been developed and the behavioral outcomes range from highly effective anxiolytic effects to no effect $(231,251,252)$. Results are inconsistent overall and thus difficult to interpret. Variation could be due to factors such model used to test anxiety or depression, route of administration or doses used.

\subsection{5. $\mathrm{MCH}$ in drug addiction}

$\mathrm{MCH} 1-\mathrm{R}$ antagonism has been extensively studied in preclinical models and clinically evaluated as a potential treatment for obesity (253). Only a handful of studies have evaluated its potential for addiction (254). MCH1-R has recently been shown to play a role in cocaine reward. Mice with genetic deletion of MCH1-R self-administer less cocaine and show less cocaine-induced CPP than their wild type controls and do not exhibit cue-induced reinstatement to cocaine (255).

$\mathrm{MCH}$ potentiates dopamine signaling and enhances action potential firing in the NAc and results in a high degree of phosphorylation of dopamine and cyclic adenosine monophosphate phosphoprotein $32 \mathrm{kDa}$ (DARPP32). This supports an important role for $\mathrm{MCH}$ in interacting with the mesolimbic dopaminergic system to regulate cocaine reward $(255,256)$. However, a different study reported no difference between MCH1$\mathrm{R}$ knockout mice and wild type controls in cocaine- or amphetamine-induced CPP 
(256). This discrepancy could be due to differences in dose, background strain or behavioral protocol.

Looking at the role of $\mathrm{MCH} 1-\mathrm{R}$ in alcohol drinking, $\mathrm{MCH}$ administration increases alcohol-intake in rats, while administration of an $\mathrm{MCH} 1-\mathrm{R}$ antagonist has no effect (257). However, $\mathrm{MCH} 1-\mathrm{R}$ knockout mice drink more alcohol than wild type controls, although the concentration of alcohol used in this study was low enough and possibly be pharmacologically inactive (258). As with cocaine the effect of $\mathrm{MCH}$ on alcohol reward is unclear and further studies are needed to clarify the direction of the effect.

This thesis focuses on the role of $\mathrm{MCH} 1-\mathrm{R}$ using an antagonist in the regulation of caloric-intake and motivation to consume alcohol in states of escalated consumption using two-bottle free choice preference drinking (paper IV). 


\subsection{Animal models of alcohol use disorder}

Understanding the brain processes underlying AUD is important and challenging. Although there has been significant progress in clinical research with technology such as functional magnetic resonance imaging (fMRI) and positron emission tomography (PET) there remain limitations in our ability to causally link neurochemical processes and signaling events in the brain to behavioral outcomes $(259,260)$. Use of experimental animal models can help provide some of the answers to these research questions.

The complexity of AUD make it difficult to model this condition in animals but nevertheless animal models are useful tools to better understand the behavioral and neurobiological mechanisms in the development and progression of alcohol dependence. Operant self-administration of alcohol and two-bottle free choice preference drinking for example can be used to study the initiation, progression and escalation of drinking, increased motivation to obtain alcohol and relapse to alcoholseeking behavior called reinstatement in animal models. These types of studies would not be ethically feasible or even possible to conduct in humans. Thus, animal models are required to screen for possible new treatment targets and to fully understand the mechanisms behind AUD.

Experimental animal models can be broadly divided into three groups that follow the progression to AUD. These are binge/intoxication, withdrawal/negative affect and anticipation/preoccupation/craving. Binge/intoxication models include operant alcohol self-administration, two-bottle free choice preference drinking and conditioned place preference. Withdrawal/negative affect models include hangover anxiety and increased motivation to obtain alcohol in a dependent state that can be induced by alcohol vapor exposure or prolonged IA to alcohol. Finally, anticipation/preoccupation/craving models includes cue- and stress-induced reinstatement of alcohol-seeking behavior $(37,54)$. Research and animal models focusing on these three key stages of AUD has been used to screen several promising targets for possible new treatments for AUD (Figure 3).

\subsubsection{Ethical issues in animal research}

The experiments presented in this thesis were conducted according to the guidelines for the care and use of laboratory animals at the National Institutes of Health or Linköping University depending on where the experiment was done. When animals are used for the benefit of humans, in our case for alcohol research it is critical that this is done in the most humane manner possible. We are unlikely to understand complex human diseases and how to treat them without the use of experimental animals. All of the experiments presented here were designed considering the " 3 Rs", a humane approach to animal research that was first proposed by Russell and Burch in 1959 and which formed the basis for laws regarding the ethical treatment of animals in many countries $(261,262)$. The 3 Rs are reduction, replacement and refinement. 
Reduce the number of animals tested or obtain more information from the same animal. Here, we used latin square design whenever possible when testing drugs such that each animal received several treatments served as its own control. This is also beneficial for the experimental design.

Replace the animal models with other alternative methods or techniques such cell culture, computational and mathematical modelling and the systems biology approach whenever possible to answer the research question.

Refine the methods to reduce or eliminate discomfort, pain and distress for the animals. Researchers should design the experiment with animal welfare in mind.

\subsubsection{Utility and validity of animal models}

Brain structure and function vary along the phylogenetic tree with one of the most obvious differences being the increasing degree of migration of function from subcortical centers to the cortex or corticalization from rodents to primates (263). However, much of the fundamental neurocircuitry is conserved between species, allowing insights to be gained from experiments using rodent models. Rats have conventionally been used in preclinical neuropsychopharmacology research due to the reliability and relative ease of invasive techniques such as microinjections, easy drug delivery and catheter placement and other microsurgeries in combination with a wide range of well-established behavioral paradigms and methods. The use of mice in neuropsychopharmacology research has also markedly increased recently. This is due to the development, application and incorporation of advanced molecular techniques such as gene targeting which offers a unique opportunity to easily study the roles of specific gene targets in behaving animals. It should also be noted that the use of mice has also practical and economic advantages with in terms of housing and breeding space (264).

The mouse is not of course a miniature human. It is impossible to fully model the complexity of human neuropsychiatric diseases in experimental animal model or to receive subjective feedback from the animal. The utility and validity of an animal model must be evaluated based on objective criteria. Some criteria for the validity of approaches to model human behavior in experimental animals have been proposed by Robbins and Sahakian in 1979 (265).

Face validity means that the behavior of the animal being tested resembles the human symptoms being modeled.

Construct validity refers to how well the theoretical basis for the model corresponds to the human condition. This cannot be established in a single experiment but rather by cumulative accumulation of the results from many studies done by different researchers. 
Predictive validity refers to as the degree to which a particular measure is able to reliably predict future observations. The most common type of predictive validity in preclinical research using animal models is pharmacological validity which means that the effect of a particular drug in an animal model reliably predicts an effect of that drug in humans.

\subsubsection{Anxiety-related behaviors}

Anxiety is activation of fear in the absence of an actual threat. Generalized anxiety disorder symptoms includes and is associated with restlessness, being easily fatigued, difficulty in concentrating or having one's mind go blank, irritability, muscle tension, difficulty controlling worrying thoughts and sleep disruptions. However, nonpathological fear in response to a threat and pathological anxiety share the same neurobiological systems. Rodent models of anxiety are based on their natural behavioral patterns. They avoid exposure to predators and thus they have innate aversion to exposed and well-lit areas, although foraging for food requires exploration of the environment which is vital for their survival so they must judge when it is safe to explore or not and there is individual variation in this (266).

In the context of behavioral pharmacology research exploration-based tasks take advantage of the conflicting tendencies to approach and avoid. In the novel open-field test the aversive area is the region area of a brightly lit open arena (267); in the elevated plus-maze it is the open elevated arms or the open center (268); in the elevated zero-maze it is the open elevated quadrants (269) and in the light-dark exploration task the brightly lit compartment (270). High levels of exploration in an aversive environment are generally interpreted as a low level of anxiety. As many anxiety disorders are characterized by avoidance of perceived threats these types of tests have high face validity. In addition, several of these have been extensively reported to have predictive validity in terms of significant reduction of avoidance behavior using drugs such as benzodiazepines that are effective as treatments for anxiety in humans with no effect of psychotropic drugs that do not modulate anxiety in humans.

These tests based on approach/avoidance behavior can be confounded by defects in sensory, motor or neurological function (271). Gene mutation can impair contextual, tactile and visual perception and thereby affect performance in behavioral tests. Measurement of general locomotor activity for example entries into closed arms of the elevated plus-maze or in a familiar environment such as the home cage can provide useful information on how to interpret behavioral data (272).

Another alternative is the avoidance test which has been developed and as a punishment-based conflict paradigm. The most common version is the Vogel conflict test for rats. Mildly water-deprived rats are provided with water from a metal spout that is occasionally paired with a mild electric shock while the rat drinks. Drugs that are 
anxiolytic in humans such as diazepam will dose-dependently attenuate the shockinduced suppression of drinking. This task can be also be adapted for mice (273).

The behavioral tasks discussed above all rely on unconditioned behaviors. The fear conditioning paradigm in contrast is based on Pavlovian conditioning from early 1900 (274). It measures fear behavior induced by exposure to a previously neutral stimulus such as a tone that has been paired with an aversive stimulus such as footshock. The dependent variable is usually the amount and degree of freezing. This behavioral paradigm has been critical for mapping out the circuitry that modulates fear learning and its associated memories (275).

\subsubsection{Depression-related behaviors}

One of the symptoms of major depression is inability to cope with stress. Several behavioral models for depression are based on exposure to inescapable stress.

The FST also known as Porsolt's test is the most widely-used screening test for antidepressant activity (276). It is based on the behavioral despair that the rodent experiences when it is placed in a confined space filled with water. The animal will initially swim vigorously and try to escape. After several attempts and a variable interval of time this escape behavior will cease and the animal will remain immobile and float. Reduction of immobility time is observed following pretreatment with an antidepressant.

Another related paradigm is the tail suspension test in which the rodent is hung or suspended upside down by its tail (277). Similar to the FST it will exhibit passive immobility after some time of struggling and attempting to escape. This test has also been shown to have predictive validity with antidepressants that are effective in humans. A limitation for this and the FST is that the behavior is sensitive to acute antidepressant effect whereas chronic treatment with antidepressants is required for therapeutic efficacy in humans.

Novelty-induced hypophagia refers to the increased latency to eat a familiar food in a novel environment (278). This paradigm is sensitive to chronic but not to acute or subchronic antidepressant treatment (279) matching results in human patients more closely.

Sucrose preference is another behavioral assessment in rodent models of depression. Rodents are naturally interested in sweet-tasting foods or drinks and reduced preference for sucrose represents anhedonia that can be reversed with antidepressant treatment. This test can measure the affective state and motivation of the animal.

The unpredictable chronic mild stress paradigm is another model for depression in rodents. This method is based on exposure to repeated unpredictable stressors such as wet bedding, disrupted light/dark cycle, food and water deprivation (280). This 
procedure has been shown to induce neurochemical and behavioral changes that resemble those observed in depressed human patients (281).

Chronic social defeat stress (SDS) is another model for depression with high face validity. Human who have experienced social rejection show increased depression, loneliness, anxiety, social withdrawal and low self-esteem (282). The SDS model could be useful to study certain depression phenotypes since many stressful stimuli that lead to psychopathological changes in humans social in nature. To subject a male rodent to SDS he is introduced into the home cage of different unfamiliar conspecific males each day as an intruder and the resident male investigated, attacks and defeats him. This causes behavioral changes such as decreased social interaction and lack of interest in previously reinforcing activities which also occurs in depression for humans. Thus this model has good face and construct validity (283).

These models for depression have also been used also to evaluate alcohol consumption and novel treatments for AUD as stress, anxiety and depression are closely associated with AUD.

\subsubsection{Alcohol-related behaviors}

Operant and non-operant self-administration paradigms have been widely used in the assessment of rewarding effects of alcohol in rodents. Rodents will spontaneously consume alcohol although consumption varies significantly between strains. The twobottle free choice drinking paradigm is a widely used and simple method to assess voluntary non-operant drinking. This method consists of free access to and choice between a bottle of water and a bottle of alcohol at various concentrations (284). Bottles can be available either under continuously or under an IA schedule. IA drinking has been shown to escalate consumption faster and higher compared to continuous access drinking $(285,286)$. Concentrations of alcohol between $10-20 \%(\mathrm{v} / \mathrm{v})$ have been used. The alcohol concentration is often increased gradually. The target concentration of alcohol can also be offered from the beginning. Animals initially need time to habituate to the taste of alcohol. Drinking is measured by weighting the bottles and the animal every day and calculating intake as grams of alcohol consumed per kilogram of body weight. The position of the bottles is changed every other day to avoid a side preference, since rodents have a high tendency to develop one. Alcohol consumption at higher concentrations can be driven by the pharmacological and reinforcing effects of alcohol while consumption at lower concentrations can be driven by taste or calories (287).

Rodents will readily work for alcohol in an operant self-administration paradigm and the extent to which they will work varies by strain. Operant paradigms require extensive training since they require a behavioral response usually pressing a lever to earn a reinforcer. Operant tasks are thought to more directly engage motivation than two-bottle free choice drinking. Training usually starts with a very low concentration of the non-caloric sweetener saccharin under mild water deprivation condition. Upon 
establishment of lever-pressing to earn the reinforcer the concentration of alcohol is slowly increased and the concentration of saccharin is slowly decreased. Operant selfadministration has been also extensively used to evaluate motivation using progressive ratio responding, as a model for relapse using cue- and stress-induced reinstatement of level pressing and modified to model compulsive behavior using quinine adulteration of the alcohol solution or electric footshock to modify alcohol seeking.

In humans reduced sensitivity to the sedative and acute ataxic effects of alcohol is heritable and contributes to genetic risk for developing AUD (288). There are several rodent models for sensitivity to the acute effects of alcohol for example loss of righting reflex (LORR). The observed effects are dose-dependent such that a low dose of alcohol $(1 \mathrm{~g} / \mathrm{kg})$ stimulates locomotor activity, a moderate dose $(2 \mathrm{~g} / \mathrm{kg})$ induces ataxia and a higher dose (3-4 g/kg) produces deep sedation and sleep. Varying pharmacological pretreatment or history with alcohol metabolism can affect the outcome.

\subsubsection{Compulsive alcohol seeking and related behaviors}

Compulsive and uncontrolled alcohol use and alcohol-seeking behavior are fundamental to addictive behavior and part of the diagnostic criteria for AUD in DSMV. These behaviors include strong craving, compulsive alcohol use, difficulty controlling craving and continued use of alcohol despite negative and harmful consequences (289). In animal models the loss of control over alcohol use in operant self-administration can be evaluated using conflict paradigms. An aversive stimulus is introduced simultaneously with the self-administered alcohol thus devaluating the positive reinforcing value of it. This can be mild electric foot shock upon responding for alcohol or taste adulteration of the alcohol solution using bitter quinine (290). It must be noted that alcohol, unlike most other drugs of abuse is a weak reinforcer and requires extensive training and lengthy exposure to induce an addiction phenotype. Animal models using long-term IA to voluntary alcohol drinking or alcohol vapor exposure may be the most useful and more for studying the transition from controlled to compulsive behavior $(291,292)$.

One factor that potentially affects alcohol drinking is alcohol withdrawal. In rodents with long-term access to alcohol followed several days/weeks/months of abstinence re-presentation of alcohol leads to a robust temporary increase in alcohol-intake compared with baseline levels. This is called the alcohol deprivation effect (ADE) and is also associated with higher breakpoint in lever-pressing in the progressive ratio test and a preference shift toward more concentrated alcohol solutions. ADE can be used as a model for relapse in terms of directly evaluation drinking behaviors after a period of protracted abstinence (293). This effect has also been observed in humans and thus the model has high face and construct validity. Furthermore, the ADE model has been pharmacologically validated using naltrexone and acamprosate to treat AUD patients (294). 


\subsubsection{Reward- and aversion-related behaviors}

The conditioned place preference (CPP) or conditioned place aversion (CPA) paradigm is a widely used rodent model for studying several rewarding and aversive properties and effect of drugs and other stimuli. The stimulus being studied is associated with distinct contextual cues (visual, tactile, olfactory and auditory) and the animal's degree of preference for or aversion to those cues is later measured. Drugs of abuse usually induce CPP though this can vary depending on dose, amount of conditioning, species, strain and route of administration. A major advantage of the paradigm is that it requires much less time and training to assess the rewarding or aversive effect of a drug compared with the self-administration paradigm (295). 


\section{OVERALL AIM OF THE THESIS}

The overall aim of these research projects were to evaluate the nociceptin/orphanin FQ (NOP) receptor, neuropeptide Y-Y2 (NPY-Y2) receptor and melanin-concentrating hormone $(\mathrm{MCH})$ receptor system as novel and potential pharmacological treatment targets for AUD using NOP receptor agonist, SR-8993; NPY-Y2 receptor antagonist, CYM-9840; and $\mathrm{MCH} 1$ receptor antagonist, GW803430.

\section{Specific aims for paper I-IV}

I. Is the novel small molecule NOP-agonist SR-8993 able to reduce alcohol-intake, attenuate relapse to alcohol-seeking, without inducing adverse side-effects such as locomotion and sedation in the alcohol-naïve state?

II. Can the findings in the alcohol-naïve state be translated over to the postdependent state and to evaluate the efficacy of SR-8993 in suppression of alcohol-intake in escalated animals during the progression from the alcoholnaïve to the high-intake state?

III. To evaluate effects of the novel NPY-Y2 antagonist CYM-9840 on motivation to self-administer alcohol and relapse like behaviors in the alcohol-naïve state.

IV. To investigate the role of $\mathrm{MCH} 1-\mathrm{R}$ and to evaluate the antagonist GW803430 in the regulation of caloric-intake and motivation to consume alcohol in states of escalated alcohol consumption 


\section{MATERIAL AND METHODS}

This section of the thesis will primarily cover and describe the animals used in the studies and the drugs (NOP agonist, NPY-Y2 antagonist and MCH1-R antagonist) that has been used for the treatments. Secondly, it will cover the preclinical models, behavioral paradigms and molecular techniques used in the experiments for the data collection. Additional detailed description of the materials and methods used can be found in the papers/manuscripts.

\subsection{Animals}

In paper I-IV male Wistar rats (250-300 grams at the start of the experiment) were used throughout. The experiments for these studies were performed at Linköping University were purchased from Taconic (Ejby, Denmark); Harlan (Horst, The Netherlands) or Charles River (Erkrath, Germany). For some of the experiments in paper I \& IV performed at the NIAAA, NIH (Bethesda, Maryland) male Wistar rats (250-300 grams at the start of the experiment) were used and were purchased from Charles River Laboratories (Wilmington, MA, USA).

Self-administration animals were group housed (3-4 per cage) for self-administration and other behavioral experiments; and single housed for two-bottle free choice drinking experiments in a temperature and humidity controlled vivarium maintained on a reverse 12:12 hour light-dark cycle with lights off at 8:00 a.m. Food and water were available ad libitum, except during the initial operant training period the rats were mildly water deprived. Rats were habituated to the animal facility for at least one week after their arrival and subsequently habituated to handling before each experiment started.

Male C57BL/6 mice from Taconic (Ejby, Denmark) weighed 19-22 grams at the beginning of the experiment. Beta-arrestin knockout mice a generous gift from Professor Robert J. Lefkowitz (Duke University, Chapel Hill, NC, USA) were initially generated on a C57BL/6 background and subsequently bred from heterozygous matings at the Center for Biomedical Resources (CBR) animal facility at Linköping University (Linköping, Sweden). Mice were grouped housed (2-4 per cage) and maintained on a 12:12 hour light-dark cycle with lights off at 7:00 p.m. in a temperature and humidity controlled vivarium with food and water available ad libitum and were tested at 8-12 weeks old. Ear punches were obtained for genotyping and the mice were identified by ear marks.

\subsection{General behavioral procedures}

For most of the behavioral procedures were appropriate the animals were allowed to acclimatize to the test room for at least half an hour prior to the testing. All testing equipment were regularly cleaned with $10 \%$ alcohol and water. All the studies, procedures and its experimental protocols was designed according to the guidelines of and approved by, the local ethics committee on animal research in Linköping University, Sweden and following the NIAAA, NIH, USA and EU guidelines. 


\subsection{Drugs}

In paper I \& II, the NOP receptor agonist (SR-8993) used was developed, synthesized and provided by the laboratory of Professor Claes Wahlestedt (University of Miami, Florida, USA). It is a small non-peptide molecule that is orally available and crosses the blood brain barrier. SR-8993 for systemic administration was dissolved in 10\% DMSO, 10\% Tween-80 and sterile water; and administered intraperitoneally (i.p) 45 minutes before testing at doses of $0.3,1$, or $3 \mathrm{mg} / \mathrm{kg}$.

In paper III, the NPY-Y2 receptor antagonist (CYM-9840) used was developed, synthesized and provided by the laboratory of Professor Edward Roberts (Scripps Research Institute, California, USA) and Professor Claes Wahlestedt (University of Miami, Florida, USA). It is a small non-peptide molecule that is orally available and crosses the blood brain barrier. The NPY-Y2 receptor antagonist was dissolved in 10\% DMSO, 10\% Tween-80 and sterile water; and administered i.p 45 minutes before testing at doses of 3 or $10 \mathrm{mg} / \mathrm{kg}$.

In paper IV, the MCH1-R antagonist (GW803430) used was developed, synthesized and provided by Lilly Research Laboratories (Indianapolis, IN, USA). It is a small nonpeptide molecule that is also orally available and crosses the blood brain barrier. GW803430 was suspended in 10\% Tween-80 and sterile water; and administered i.p 45 minutes before testing at doses of 3,10 , or $30 \mathrm{mg} / \mathrm{kg}$.

\subsection{Elevated plus-maze}

The elevated plus-maze (EPM) is a well-established and pharmacologically validated model of anxiety in rodents (268). We evaluated the effects of SR-8993 (in paper I) and CYM-9840 (in paper III) respectively on the EPM. The apparatus is constructed of black plastic and consists of two $30 \times 10 \mathrm{~cm}$ closed arms with $40 \mathrm{~cm}$ high nontransparent side walls across from each other and two open arms of the same dimensions with no side walls across from each other, in a plus-sign formation. The EPM was placed $50 \mathrm{~cm}$ above the floor during the testing and the tests were conducted under dim red light. Prior to testing, each animal was placed in a new, clean home cage for five minutes to enhance exploratory activity and thereby improve the reliability of subsequent plus-maze testing. At the beginning of the test, the rat was placed on the central platform facing one of the open arms. Behavior was observed and scored for 5 minutes. Entry into an arm was operationally defined as all four paws entering into that arm. The number of entries into open and closed arm; and the time spent in open and closed arms were scored manually or using Noldus Ethovision XT tracking software (Noldus, Wageningen, The Netherlands). The maze was cleaned with $10 \%$ alcohol solution and completely dried between tests. Dependent variables were \% time spent in the open arms and \% entries into the open arm, calculated as open/(open + closed $)^{\star} 100$. The number of entries into the closed arms was used as an index of overall locomotor activity (296). 


\subsection{Acute alcohol withdrawal-induced anxiety}

The EPM was also used as a model to score the alcohol withdrawal-induced "hangover" anxiety. In this case, rats received an i.p injection of alcohol (3 g/kg, 20\% $\mathrm{v} / \mathrm{v}$ ) or saline 12 hours prior to EPM. The animals were treated with the drug or vehicle i.p 45 minutes before testing. The procedure was the same as described above. We evaluated the effects of SR-8993 (in paper I) and CYM-9840 (in paper III) respectively on acute alcohol withdrawal-induced anxiety.

\subsection{Open-field}

The open-field test measures general locomotor activity and can also be used as a model of anxiety (267). We evaluated the effects of SR-8993 (in paper I) and CYM9840 (in paper III) respectively in the open-field. The apparatus consisted of a $45 \mathrm{x}$ $45 \mathrm{~cm}$ box with a $50 \mathrm{~cm}$ high walls placed on the floor, with lines dividing it into center and peripheral sections. Testing was conducted under dim white light for 15 minutes, beginning with placing the rat in the center. Behavior was recorded with a video camera and subsequently scored using Noldus Ethovision XT tracking software (Noldus, Wageningen, The Netherlands) or by using automated open-field boxes from Med Associates Inc., (Georgia, VT, USA) which uses infra-red beams to detect the activity. Exploration of the center of the apparatus indicates lower anxiety while exploration of the periphery indicates higher anxiety. Animals were first habituated to the open-field with no treatment for 10 minutes to reduce novelty effects and then tested the following day after treatment with drug. The apparatus was cleaned with $10 \%$ alcohol and dried between subjects.

\subsection{Forced swim test}

The forced swim test (FST) is a well-established and pharmacologically validated model of depression in rodents (276). We evaluated the effects of SR-8993 (in paper I) and CYM-9840 (in paper III) respectively using FST. Since anxiety and depression are closely related, it is important to include a measure of depression in studies examining changes in anxiety-related behaviors. The apparatus consists of a white plastic tub with diameter $34 \mathrm{~cm}$ and height $66 \mathrm{~cm}$ that is filled with water to a level of $45-48 \mathrm{~cm}$. The temperature of water was maintained between $22-25^{\circ} \mathrm{C}$. Animals were habituated to the apparatus with no treatment for 10 minutes on the pre-test day and then tested the following day for 5 minutes after treatment with the drug. Behavior was recorded using a video camera and later scored manually. Dependent variables were latency to immobility and time spent immobile. After the test, animals were removed from the water, dried with a towel, placed in a cage that was heated from below (half on/half off a heating pad) until fully dry and then returned to the home cage. 


\subsection{Operant alcohol self-administration}

Operant conditioning chambers housed in sound attenuating cubicles were equipped with two retractable levers positioned on either side of a drinking reservoir. Visual stimuli were presented via a light located on the back panel. A microcomputer controlled delivery of fluids, presentation of visual stimuli and recording of behavioral output. Rats were trained to self-administer $0.2 \%(\mathrm{w} / \mathrm{v})$ saccharin in 30 minute daily session on a fixed ratio 1 (FR1) schedule of reinforcement with each active lever response resulting in delivery of $0.1 \mathrm{ml}$ of the saccharin solution. After each reinforcer delivery, there was a 5 second time-out period during which additional active lever presses were recorded but resulted in no reinforcer delivery. Inactive lever presses had no programmed consequences. After one week of saccharin self-administration, rats were trained to self-administer $10 \%(\mathrm{v} / \mathrm{v})$ alcohol by increasing the alcohol concentration from $2 \%-4 \%-6 \%-8 \%-10 \%$ and gradually fading out the saccharin over two weeks. Rats were then trained to self-administer $10 \%(\mathrm{v} / \mathrm{v})$ alcohol in 30 minutes daily session on an FR1 schedule for 2-3 weeks until they reached a stable baseline. Next, the effect of the drugs on alcohol self-administration was assessed using a latin square counterbalanced within-subjects design with one week between test sessions, with drug or vehicle administered i.p 45 minutes before the session. After each test session, rats continued daily self-administration sessions over the following week to reestablish baseline responding. We evaluated the effects of SR-8993 (in paper I \& II), CYM-9840 (in paper III) and GW803430 (in paper IV) respectively in the selfadministration model.

\subsection{Progressive ratio responding for alcohol self-administration}

Rats were trained to self-administer $10 \%(\mathrm{v} / \mathrm{v})$ alcohol as described above. When stable responding was reached, rats were tested for progressive ratio (PR) responding. The progression of lever presses required to receive an alcohol delivery was $1,2,3,4,6,8,10,12,16$ and then increases in steps of four until the end of the test session (approximately 2 hours). The breakpoint was defined as the last ratio completed when 30 minutes had passed before the rat pressed the active lever enough times to reach the next level. We evaluated the effects of SR-8993 (in paper I) and CYM-9840 (in paper III) respectively in the PRR. 


\subsection{Cue- and stress-induced reinstatement of alcohol seeking}

Rats were trained to self-administer $10 \%(\mathrm{v} / \mathrm{v})$ alcohol as described above. For cueinduced reinstatement, an orange-scented contextual cue was paired with alcohol available during 2 weeks of baseline sessions. After stable responding was reached, rats underwent 14 daily extinction sessions during which both alcohol and the contextual cue was absent. Once the extinction criterion of fewer than 10 lever presses per 30 minutes session for three consecutive sessions was reached, animals were randomly assigned to treatment with vehicle or drug, administered i.p 45 minutes before the 30 minutes reinstatement test. During the reinstatement test, the contextual cue was present but alcohol was not available. For stress-induced reinstatement, rats were trained to self-administer $10 \%(\mathrm{v} / \mathrm{v})$ alcohol with no additional contextual cues present, underwent extinction as described above and were administered yohimbine $(1.25 \mathrm{mg} / \mathrm{kg})$ a pharmacological stressor 5 minutes before drug or vehicle administration for the reinstatement test. We evaluated the effects of SR-8993 (in paper I) and CYM-9840 (in paper III) respectively using this reinstatement models.

\subsection{Two-bottle free choice drinking (4 hours limited access)}

Rats were single housed in order to measure individual intake with 4 hours limited access to alcohol solution in the beginning of the dark cycle. Alcohol concentration was gradually increased from $3 \%-6 \%-9 \%(\mathrm{v} / \mathrm{v})$. The position of the bottles was changed daily to avoid place preference for a particular side. After establishing stable baseline drinking for 12 weeks, rats were treated with the drugs. Alcohol and waterintake were recorded after the drug administration and body weight was recorded on the day of treatment and the day after. Alcohol-intake $(\mathrm{g} / \mathrm{kg})$ was calculated by multiplying volume of alcohol consumed $(\mathrm{ml})$ by concentration of alcohol $(\mathrm{v} / \mathrm{v})$ given and density of alcohol $(0.7893 \mathrm{~g} / \mathrm{ml})$ and dividing by body weight $(\mathrm{kg})$. Alcoholpreference expressed as percentage (\%) was calculated by dividing volume of alcoholintake by volume of total fluid-intake and multiplying by 100 . We evaluated the effects of SR-8993 (in paper I) and CYM-9840 (in paper III) respectively using this drinking model.

\subsection{Two-bottle free choice drinking (Intermittent Access)}

IA was performed using a two-bottle free choice procedure with access to water and $20 \%(\mathrm{v} / \mathrm{v})$ alcohol from the beginning of the experiment without a gradual increase in concentration. Following intermittent exposure for 11-15 weeks in order to induce escalation of alcohol-intake and establishment of stable drinking baseline. Upon drug treatment alcohol and water-intake were recorded 1, 2, 4 and 24 hours post treatment. Alcohol-intake and preference was calculated using the formula mentioned above. We evaluated the effects of SR-8993 (in paper I \& II) and GW803430 (in paper IV) respectively using this drinking model. 


\subsection{Operant alcohol self-administration following intermittent} access induced escalation

Rats were trained to self-administer alcohol as described above. After a stable baseline was established, rats were single housed and given access to $20 \%(\mathrm{v} / \mathrm{v})$ alcohol intermittently on Mondays, Wednesdays and Fridays; with only water available on the other days of the week. After a long period of IA drinking, animals were retrained to self-administer of $20 \%(\mathrm{v} / \mathrm{v})$ alcohol shortly to access the new baseline. Then tested on progressive ratio and finally treated with drug. We evaluated the effects of SR-8993 (in paper I \& II) and GW803430 (in paper IV) respectively using this model. Separate group of rats were generated using this procedure for the gene expression studies (in paper IV).

\subsection{Operant alcohol self-administration after intermittent alcohol} vapor exposure

Intermittent alcohol vapor exposure by evaporating alcohol into the air that are used to ventilate the cages 14 hours per day and the remaining 10 hours of the day no alcohol was present in the air. 5 days per week for 7 weeks was utilized to induce dependence. This schedule of exposure has been optimized to generate a reliable blood alcohol concentration (BAC $\geq 150 \mathrm{mg} / \mathrm{dl}$ ) and still produce daily withdrawal. Blood samples were taken once per week for BAC analysis. After 7 weeks of alcohol vapor exposure rats were trained to self-administer alcohol. When stable baseline responding for alcohol was established, rats were treated with the drug or vehicle using a latin square within-subjects design or used for gene expression studies. We evaluated the effects of GW803430 (in paper IV) using this model.

\subsection{Loss of righting reflex}

Rats were administered $3 \mathrm{~g} / \mathrm{kg}$ of $20 \%(\mathrm{v} / \mathrm{v}$ ) alcohol i.p and placed in a supine position upon loss of consciousness. Time to regain righting reflex, defined as the ability to turn over into the upright position after being manually placed back in the supine position 3 times within 1 minute was measured. BAC upon sleeping and waking was also measured. We evaluated the effects of SR-8993 (in paper I) and CYM-9840 (in paper III) respectively using this model.

\subsection{Taste-preference}

Taste-preference was tested using two bottle free choice drinking with continuous access to water and $0.2 \%$ saccharin solution or $0.001 \%$ quinine solution, as previously described by Goodwin and Amit et. al 1998. Once drinking was stable rats were treated with vehicle or drug and their drinking was measured for the following 24 hours. We evaluated the effects of SR-8993 (in paper I) and CYM-9840 (in paper III) respectively using this model. 


\subsection{Blood alcohol concentration}

The blood alcohol concentration (BAC) was determined by a coupled enzyme reaction that resulted in a colorimetric $(570 \mathrm{~nm})$ product proportional to the amount of alcohol present. Standards for colorimetric detection were generated as follows: $50 \mu \mathrm{l}$ of 17.15 $\mathrm{N}$ alcohol diluted with $808.7 \mu \mathrm{l}$ alcohol assay buffer to generate a $1 \mathrm{mmol} / \mu \mathrm{l}$ standard, $10 \mu \mathrm{l}$ of the $1 \mathrm{mmol} / \mu \mathrm{l}$ standard solution diluted with $990 \mu \mathrm{l}$ alcohol assay buffer to generate a $10 \mathrm{nmol} / \mu \mathrm{l}$ standard, $100 \mu \mathrm{l}$ of the $10 \mathrm{nmol} / \mu \mathrm{l}$ solution diluted with $900 \mu \mathrm{l}$ alcohol assay buffer to generate a $1 \mathrm{nmol} / \mu \mathrm{l}$ standard. $0,6,12,18,24$ and $30 \mu \mathrm{l}$ of the of the $1 \mathrm{nmol} / \mathrm{\mu l}$ standard solution were added to wells in a 96 -well plate and alcohol assay buffer was added to each well to a final volume of $50 \mu$ per well, generating 0 (blank), 2, 4, 6, 8and $10 \mathrm{nmol} /$ well standards. Samples were added to wells and diluted in alcohol assay buffer to a final volume of $50 \mu \mathrm{l} .50 \mu \mathrm{l}$ of the master reaction mix (46 $\mu \mathrm{l}$ alcohol assay buffer, $2 \mu \mathrm{l}$ alcohol probe, $2 \mu \mathrm{l}$ alcohol enzyme mix) was added to each well. Samples were mixed with a horizontal shaker and incubated for 30 minutes at $37^{\circ} \mathrm{C}$. Absorbance was measured at $570 \mathrm{~nm}$ (A570). The background value obtained for the 0 (blank) alcohol standard was subtracted from all other readings. The values obtained from the standards were used to plot a standard curve. The amount of alcohol present in each sample was determined from the standard curve. We evaluated the effects of SR-8993 (in paper I) and CYM-9840 (in paper III) respectively using this method.

\subsection{Conditioned place preference}

The CPP apparatus consisted of two compartments with equal illumination and different tactile and visual cues, one with black walls and a grid floor and one with white walls and a wire mesh floor, separated by a guillotine door and equipped with infrared photo beams to determine movement and location of the mouse. The apparatus was placed in a sound attenuated chamber equipped with a fan generating white noise to mask external sound. Mice were given a 15 minutes pretest with the guillotine door open to establish that they did not have an initial side preference and those that spent more than $60 \%$ of the time on one side were excluded from the experiment. Mice were trained for four days with two 5 minutes sessions, one in the morning and one in the afternoon. One with $2 \mathrm{~g} / \mathrm{kg}$ i.p alcohol in one compartment and one with an equivalent volume i.p saline in the other compartment in a counterbalanced unbiased design with the guillotine door closed. On the test day, time spent in the alcohol- and saline-paired compartments with the guillotine door open was measured for 15 minutes. We evaluated the effects of SR-8993 (in the future perspectives section) using this model. 


\subsection{Real-time polymerase chain reaction}

Rats with high intake during 20 weeks of IA drinking were sacrificed after 1 weeks of protracted abstinence following IA; high intake $(n=7)$ and naïve controls $(n=7)$ were used in this experiment. Brains were sliced in $1 \mathrm{~mm}$ sections using a cryostat according to the Paxinos and Watson rat brain atlas (6 $6^{\text {th }}$ edition). Regions of interest were dissected by micro punches and immediately frozen on dry ice and stored at $80^{\circ} \mathrm{C}$. Tissue was homogenized by shaking with $5 \mathrm{~mm}$ stainless steel beads (Qiagen, Hilden, Germany) in a TissueLyser (Qiagen, Hilden, Germany) at $25-30 \mathrm{~Hz}$ for 2 minutes. RNA was then isolated using the RNeasy Mini kit (Qiagen, Hilden, Germany) according to the manufacturer's instructions. Total RNA concentrations were determined using the Nano Drop (ND-1000 spectrophotometer, Nano Drop Technologies, Wilmington, DE, USA). $80 \mathrm{ng}$ of total RNA from each sample was reverse transcribed into cDNA using a high capacity cDNA reverse transcription kit (Applied Biosystems, Foster City, CA, USA) according to the manufacturer's instructions. mRNA expression of the gene of interest was determined using the 7900 Fast Real-Time PCR System (Applied Biosystems). Reactions were performed in duplicate in 96-well optical plates using TaqMan Fast Universal PCR Master Mix (2x; Applied Biosystems). Beta-actin and GAPDH were used as control house-keeping genes. All probes were synthesized by Applied Biosystems. Data were acquired and processed with SDS software v2.3 (Applied Biosystems). This method was used to evaluate the expression of $\mathrm{MCH}$ and $\mathrm{MCH} 1-\mathrm{R}$ upon protracted abstinence following IA and acute withdrawal from alcohol vapor (in paper IV).

\subsection{Genotyping of beta-arrestin mice}

Mouse ear biopsies were lysed and digested in $50 \mathrm{mM} \mathrm{NaOH}$ at $95^{\circ} \mathrm{C}$ on a shaking heating block for $45-50$ minutes. Tris- $\mathrm{HCl} \mathrm{pH} 7.5$ was added to each sample to neutralize it. Samples were then vortexed, centrifuged and the supernatant was collected and stored at $-80^{\circ} \mathrm{C}$ until further use. Samples were diluted four times in DNase-free water. PCR was subsequently performed using the Clontech Advantage 2 PCR kit (Takara Bio USA, Mountain View, CA, USA) with three different primers for knockout, wild type and heterozygote genes. The PCR samples were separated on $1.5-2 \%$ agarose gels. The lower band (300-400 bp) detected on the gel represented the knockout gene, the higher band (650-800 bp) represented the wild type gene and samples with both bands represented the heterozygote. The beta-arrestin knockout mice were used to evaluate the effects of SR-8993 (in the future perspectives section) in the CPP experiment. 


\subsection{Immunohistochemistry of extracellular signal-regulated}

\section{kinase}

C57BL/6 wild-type mice were administered SR-8993 or vehicle i.p 30 minutes before i.p administration of $2 \mathrm{~g} / \mathrm{kg}$ alcohol or saline and then transcardially perfused 30 minutes after the second injection. Mice were euthanized by $\mathrm{CO}_{2}$ inhalation and quickly perfused with $36 \mathrm{ml}$ of $4 \%$ paraformaldehyde. Brains were extracted and postfixed in $4 \%$ paraformaldehyde for $2-4$ hours, transferred to $30 \%$ sucrose solution for dehydration for approximately 48 hours, snap frozen in isopentane and stored at $80^{\circ} \mathrm{C}$. Frozen $30 \mu \mathrm{m}$ sections containing the NAc (core and shell), BNST, BMA and CeA were collected in cryoprotective solution containing $20 \%$ glycerol and $30 \%$ ethylene glycol in 1x PBS using a cryostat according to the Franklin and Paxinos mouse brain atlas ( $3^{\text {rd }}$ edition) and stored at $-20^{\circ} \mathrm{C}$. Sections were washed in $1 \times$ PBS and incubated in $1 \%$ hydrogen peroxide for 30 minutes. Next the sections were transferred to $3 \%$ bovine serum albumin (BSA) for 1 hour and then incubated overnight with the primary antibody (rabbit anti-pERK, 1:300, Cell Signaling Technology, MA, USA) in $3 \%$ BSA solution at $4^{\circ} \mathrm{C}$ on a shaker. The next day, sections were washed in 1x PBS and then incubated with the biotinylated secondary antibody (anti-rabbit lgG, $1: 800$, Thermo Fisher) for 1 hour. Next the sections were washed and incubated in avidin-biotin-peroxidase complex (Thermo Fisher) for 30 minutes. Sections were then washed and stained with diaminobenzidine (DAB) chromogen (Sigma) for approximately 4 minutes, washed and mounted on gelatin-coated slides. The next day, sections were dehydrated through an alcohol-xylene series, cover-slipped and imaged using an Olympus BX51 light microscope at 20x magnification equipped with a digital camera. An experimenter blinded to treatment counted cells using ImageJ ( $\mathrm{NIH}$, Bethesda, Maryland, USA). For each subject, one section per hemisphere was quantified per brain area. The dependent variable was number of immunoreactive cells per square millimeter. We evaluated the effects of alcohol and SR-8993 on the level of phosphorylation of extracellular signal-regulated kinase (ERK) (in the future perspectives section) using this immunohistochemistry (IHC) method.

\subsection{Statistical Analysis}

GraphPad Prism (v.5.04; GraphPad software, Inc., La Jolla, CA, USA) and SPSS (v.22; IBM; Stockholm, Sweden) was used. Data were analyzed by analysis of the variance (ANOVA) followed by Newman-Keuls post hoc tests when appropriate or by Kruskal-Wallis ANOVA when data did not meet parametric assumptions. Two-way ANOVA was used to determine effects of alcohol treatment or drug and one-way repeated measures ANOVA was used to analyze the self-administration data with treatment as the factor. 


\section{RESULTS AND DISCUSSION}

This section describes and discusses the major findings in papers I, II, III \& IV accordingly in a concise form. For full details regarding results and discussions please refer to the specific paper and manuscript which are attached accordingly at the end of this book.

\subsection{The nociceptin/orphanin FQ receptor agonist SR-8993 as a candidate therapeutic for alcohol use disorders: validation in rat models (Paper I)}

\subsubsection{Acute alcohol withdrawal-induced anxiety in the elevated plus-maze was reversed by SR-8993}

In the EPM SR-8993 was mildly anxiolytic when given to naïve animals and potently reversed acute alcohol withdrawal-induced ("hangover") anxiety observed 12 hour after alcohol administration (Figure $\mathbf{5} \mathbf{A}$ and $\mathbf{B}$ ). It has been reported previously that the activity to reverse withdrawal-induced anxiety is a short term model that is very useful in drug screening and is a predictor of activity in rodent models of long term neuroadaptations induced by alcohol $(16,297)$.

A.

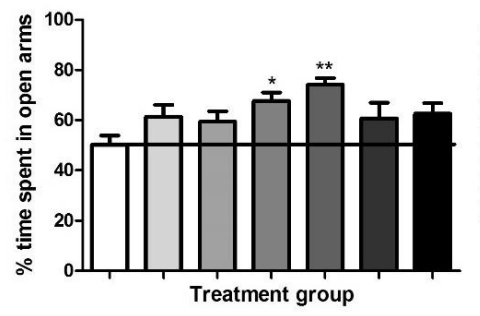

B.

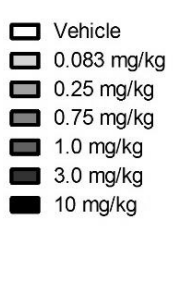

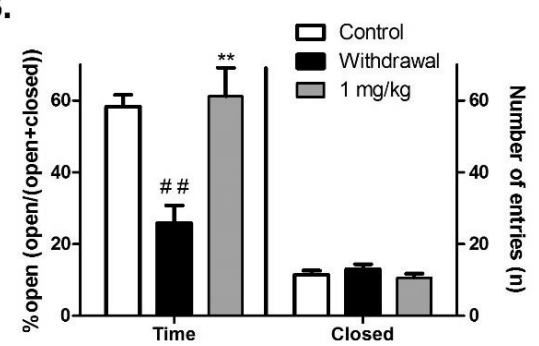

Figure 5. Behavioral screening for effective dose of NOP agonist SR-8993 using the elevated plus-maze. Elevated plus-maze during non-challenged conditions (A) and testing for reversal of alcohol-induced hangover anxiety (B). (A) Pretreatment with SR-8993 at a dose of $1 \mathrm{mg} / \mathrm{kg}$ bodyweight had a pronounced anxiolytic-like effect on the elevated plus-maze during baseline, non-stressful conditions. Data presented is percent time spent on the open arm out of time spent on any arm (100\% $\times($ open/(open + closed)). (B) The same dose was effective in reversing the anxiogenic effects of an acute administration of a high dose of alcohol. Withdrawal anxiety was induced by an acute administration of alcohol (i.p $3.0 \mathrm{~g} / \mathrm{kg}$ ) at $12 \mathrm{~h}$ prior to testing using the elevated plus-maze and the pretreatment with SR $8993(1 \mathrm{mg} / \mathrm{kg})$ was administered $45 \mathrm{~min}$ prior to plus-maze exposure. Data presented is percent time spent on the open arm out of time spent on any arm (100\% $\%$ (open/(open + closed)) as well as the number of closed arm entries, a measure of locomotor activity. All values are given as mean \pm SEM. (A) * $p<0.05$ vs. vehicle, ${ }^{* *} p<0.01$ vs. vehicle. (B) ${ }^{*} p<0.05$ vs. vehicle, \#\# $\mathrm{p}<0.01$ vs. non-alcohol control, ${ }^{* *} \mathrm{p}=0.01$ vs. withdrawal. 


\subsubsection{Operant self-administration, progressive ratio responding and cue- and stress-induced reinstatement of alcohol seeking were attenuated by SR-8993 in alcohol naïve Wistar rats}

Activation of NOP receptors by SR-8993 potently and dose dependently decreased alcohol self-administration (Figure 6 A). These results are supported by previous findings where activation of NOP receptors decreases alcohol self-administration $(141,143,298)$. The operant self-administration model better reflects motivation to obtain alcohol compared to home cage drinking which further strengthens these results.

SR-8993 significantly reduced the progressive-ratio break-points for alcohol directly reflecting reduced motivation to work for alcohol (Figure 6 B). PRR results reflect the reinforcing efficacy of drug of abuse (299-301) and thus SR-8993 leads to reduced motivation to work for alcohol.

Reinstatement of previously extinguished alcohol-seeking behavior elicited by alcoholassociated cues or by a yohimbine stressor were also significantly attenuated by NOP receptor agonism (Figure $\mathbf{6} \mathbf{C}$ and $\mathbf{D}$ ). Our findings are in agreement with previous studies showing that NOP receptors regulate the rewarding effects of various drugs of abuse $(145,302,303)$. Specifically, previous studies have shown that activation of NOP receptors reduces anxiety (142), attenuates alcohol self-administration as well as cue- and stress-induced reinstatement of alcohol seeking (143). Cue- and stressinduced reinstatement to drug seeking are mediated by distinct brain pathways that ultimately converge, with cue-induced reinstatement primarily mediated by the mesolimbic DA system and stress-induced reinstatement primarily mediated by $\mathrm{CRH}$ and noradrenaline $(304,305)$.

The finding that pretreatment with SR-8993 blocked yohimbine stress-induced reinstatement and this is supported by previous studies showing a role of the NOP system in stress responses $(139,306-308)$. It is not clear how NOP regulates stress reactivity but there is some evidence of interactions between the NOP and the CRH systems $(309,310)$. Interestingly, SR-8993 has been recently shown to be involved in sensitized stress systems in an animal model of PTSD (311).

Our results are in parallel support and expand on previous findings by Kuzmin and colleagues that NOP agonism potently and selectively suppresses alcohol selfadministration, motivation to seek alcohol, as well as cue- and stress-induced reinstatement of alcohol seeking (143). 
A.

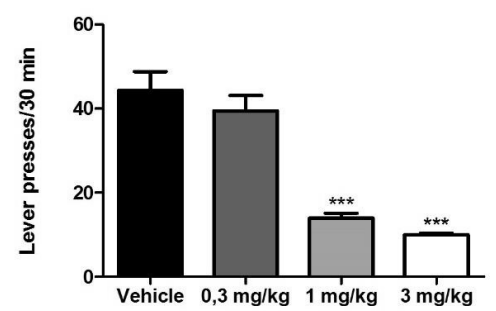

C.

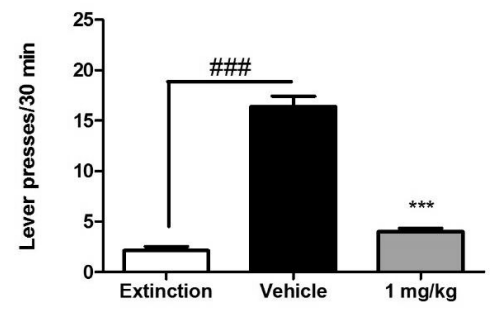

B.

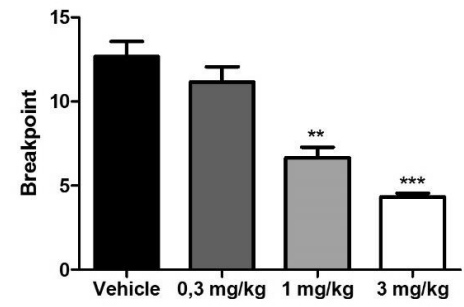

D.

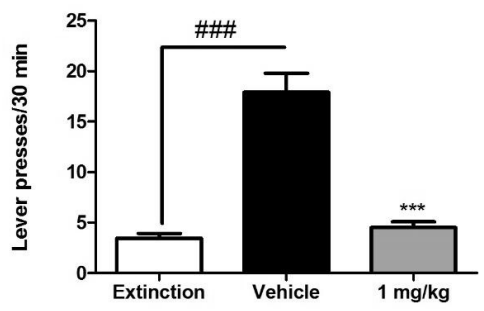

Figure 6. Effect of NOP agonist in the alcohol self-administration, progressive ratio and relapse paradigm. SR-8993 suppresses alcohol self-administration (A), decreases motivation to work for alcohol (B) and prevents both cue- (C) and stress-induced relapse-like behavior (D). (A) Operant self-administration of alcohol ( $10 \% \mathrm{~V} / \mathrm{N})$ was significantly attenuated by pretreatment with 1 and $3 \mathrm{mg} / \mathrm{kg}$ SR-8993. (B) Pretreatment with SR-8993, significantly decreased motivation to work for alcohol delivery as measured using progressive-ratio responding. Breakpoint is defined as the highest number of lever presses the animals performed to receive one dose of alcohol in the drinking cup. (C) Exposure to alcohol-associated cues (light/orange odor) induced reinstatement of lever-pressing at the lever previously paired with alcohol delivery (extinction vs. vehicle bars). Pretreatment with SR-8993 $(1 \mathrm{mg} / \mathrm{kg}$ ) completely blocked this cue-induced reinstatement (vehicle vs. $1.0 \mathrm{mg} / \mathrm{kg}$ bars). (D) Similar to what was observed for cue-induced reinstatement, yohimbine-induced reinstatement was blocked by pretreatment with SR-8993. All values are given as mean \pm SEM. ${ }^{* \star} p<0.01$ vs. vehicle, ${ }^{* \star *} p<0.001$ vs. vehicle.

\subsubsection{Alcohol-intake in the $\mathbf{4}$ hour limited access and intermittent access drinking was reduced by SR-8993}

SR-8993 significantly suppressed two-bottle free choice drinking in the 4 hour limited and IA drinking model. As previously reported IA to alcohol results in an escalation of alcohol-intake over time (286). Pretreatment with SR-8993 dose dependently suppressed alcohol-intake without affecting water-intake at the doses that reduced alcohol-intake in the IA (Figure 7 A).

It has been previously shown that NOP receptor antagonist $\mathrm{J}-113397$ facilitates the acquisition of cocaine- and morphine-induced CPP (12) and increases both alcohol and water-intake (13). It can thus be hypothesized that the endogenous NOP system inhibits drug-induced activation of the brain reward systems and that inactivation of 
this system using an antagonist renders the individual more susceptible to the rewarding effects of a drug.

Unlike NOP and other peptidergic NOP agonists like OS-462, UFP-102 and UFP-112, Ro 64-6198 a non-peptidergic NOP agonist failed to decrease voluntary alcohol-intake possibly due to the fact that the compound itself has a residual agonist activity at the mu-opioid receptors $(142,312)$.

\subsubsection{SR-8993 reduced escalated alcohol-intake in intermittent access two-bottle free choice drinking and operant self- administration in rats with a history of escalation}

Exposing rats to prolonged (for 15 weeks) two-bottle free choice IA to $20 \% \mathrm{v} / \mathrm{v}$ alcohol resulted in robust escalation of subsequent operant alcohol self-administration compared to naïve rats and pretreatment with SR-8993 significantly attenuated the escalated lever-responding (Figure $7 \mathbf{B}$ ). It has been previously reported that IA to $20 \% \mathrm{v} / \mathrm{v}$ alcohol results in a robust escalation of alcohol-intake (286).

A history of alcohol exposure results in neuroadaptive changes in the brain and escalation of alcohol-intake here is directly driven by compulsive alcohol seeking behavior in order to get relief from the negative effects during the protracted abstinence (16). NOP system is dysregulated in this phase of dependence; and in this state it has been reported that ICV administration of NOP attenuates alcohol-intake and produces anxiolytic effect as reflected in the EPM.

A.

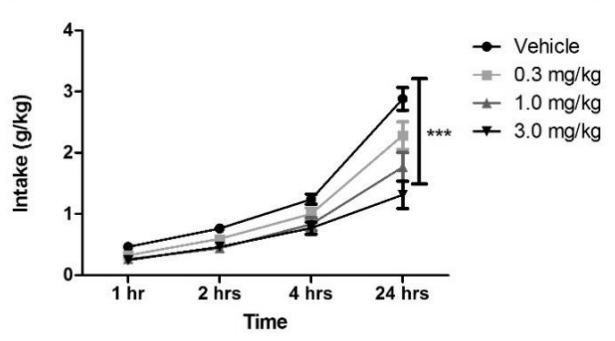

B.

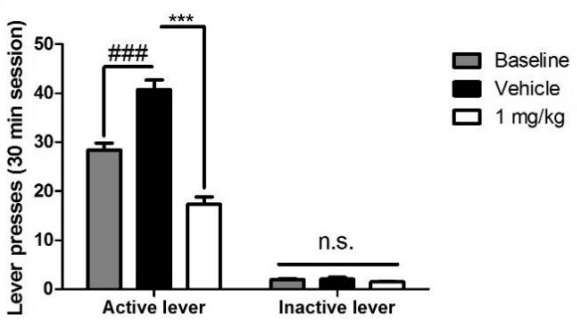

Figure 7. Effects of SR-8993 on alcohol-intake (A) and operant responding (B) in animals with escalated alcohol consumption. Animals were allowed intermittent $(24 \mathrm{~h}$, three times per week) access to $20 \% \mathrm{v} / \mathrm{v}$ alcohol for 15 weeks. (A) During the IA to $20 \% \mathrm{~V} / \mathrm{V}$ alcohol, SR-8993 at 1 and $3 \mathrm{mg} / \mathrm{kg}$ significantly attenuated consumption over the $24 \mathrm{~h}$ time period. (B) In operant self-administration, a significant escalation of responding from baseline prior to IA to re-training after the IA paradigm was seen (baseline vs. vehicle bars). Pretreatment with SR-8993 $(1 \mathrm{mg} / \mathrm{kg})$ significantly attenuated responding. Baseline indicates responding prior to 15 weeks of IA to $20 \% \mathrm{v} / \mathrm{v}$ alcohol. The line indicating "Escalation" points to responding after IA following either vehicle or SR-8993 (1 mg/kg) treatment. All values are given as mean \pm SEM. (A) ${ }^{* * *} p<0.001$ vs. vehicle, (B) \#\#\# $p<0.001$ vs. extinction, ${ }^{* * *}$ $p=0.01$ vs. vehicle. 


\subsubsection{Control experiments confirmed that the effects of SR-8993 were specific to alcohol reward and motivation to seek alcohol}

A battery of control experiments was conducted to evaluate the specificity of the effects observed after SR-8993 administration on alcohol related behaviors. Behaviors and measures such as LORR, alcohol elimination rate, basal locomotor activity, FST, taste-preference (saccharin/quinine adulteration) and $0.1 \%$ saccharin selfadministration were unaffected by pretreatment with SR-8993. Thus the effects of SR8993 observed were specific to measures of the rewarding and motivational aspects of alcohol-intake and seeking.

\subsection{Escalation of self-administration following intermittent access to alcohol selects for sensitivity to treatment with a nociceptin/orphanin FQ receptor agonist SR-8993 (PAPER II)}

IA to alcohol has been shown to lead to neurochemical adaptations in the mesolimbic system (313) that are associated with reduced dopamine output into the NAc 24 hours into withdrawal as measured by in-vivo microdialysis (313). Withdrawal from extended or chronic exposure to alcohol results in substantially decreased activity of the dopaminergic VTA neurons projecting to the NAc (314). This marked suppression of DA levels in the NAc is associated with alcohol craving during relapse (315). In the long term alcohol consumption results in an allostatic attenuation of the reward system resulting in the development of tolerance (316). This allostatic change is also responsible for the transition from positive to negative reinforcement mechanisms in addiction (317). Decrease in VTA dopaminergic neuron firing and DA release into the NAc are associated with the suppression of reward function after long-term exposure to alcohol (313), leading to alcohol-seeking behavior that is modulated mainly by negative reinforcement mechanisms. The IA model of two-bottle free choice drinking may therefore initiate allostatic changes that are accompanied by neurochemical deficiencies in the mesolimbic reward circuit and recruit mechanisms involved in negative reinforcement. It is critical that in order to model the transition from impulsive to compulsive alcohol use, a rat model includes the intermediate phase with elevated drinking behavior. This is in order to model the human condition accurately for medication development is needed for AUD (318). IA two-bottle free choice preference drinking is thus useful as a method for screening and evaluating potential pharmacological interventions. It should also noted that IA is not a pure model of dependence but rather a model of chronic, prolonged and repeated alcohol-intake model. It does not induce physical dependence to a level as such seen following alcohol vapor exposure or liquid diet, but it is useful here because we would expect a compound affecting reward-related signaling and behavior to be effective here and maybe not to be as effective in the "true" post-dependent state/phase. 


\subsubsection{A history of operant self-administration resulted in increased alcohol-intake and preference in the intermittent access two-bottle free choice model and escalated operant responding}

A history of short-term operant self-administration resulted in a rapid and significant increase of alcohol-intake and preference compared with naïve rats. There are several approaches to model escalated alcohol-intake and resulting effects such as increased stress sensitivity. These can involve passive induction of dependence (318) or IA to voluntary consumption of alcohol (286). Several studies have reported that previous experience with alcohol leads to higher initial levels of consumption and increased preference $(315,319,320)$. This supports our finding that rats with previous experience with operant alcohol self-administration consumed more alcohol and had higher preference for alcohol compared with controls in the IA two-bottle free choice preference drinking model.

\subsubsection{A robust positive correlation between alcohol-intake and preference regardless of previous experience with alcohol self-administration}

There was a robust positive correlation between alcohol-intake and alcohol-preference during IA two-bottle free choice preference drinking in both SA-trained and SAuntrained groups. Awareness of this relationship between alcohol-intake and preference in the two-bottle free choice paradigm could be useful in protocol design in operant conditioning models. Understanding how different components of motivation lead to escalation of drinking or not is useful for determination of mechanisms of action underlying AUD (321).

\subsubsection{Intermittent access to alcohol resulted in escalated alcohol self-administration and increased motivation to obtain alcohol}

The IA procedure appears useful for studying neurobiological mechanisms of alcohol consumption and preference, neuroadaptations that occur with escalated drinking and evaluating the efficacy of potential treatments. However, this method does not address the motivational and reinforcing processes that regulate alcohol-intake and seeking, which are also critical in the development of addiction. Thus we used the operant selfadministration procedure to evaluate potential shifts in motivational behavior induced by the IA procedure to better model what occurs during the development of AUD in humans $(285,318)$. We found that previous experience with operant alcohol selfadministration before IA two-bottle free choice preference drinking resulted in a faster acquisition of alcohol self-administration after the IA procedure and significantly increased lever-pressing for alcohol during self-administration compared to the nonIA controls (Figure 8 A-D). Additionally, the group with previous exposure to alcohol 
self-administration had significantly higher operant-responding after IA to alcohol drinking as compared with their pre-IA baseline.

After evaluating post-IA operant self-administration, we tested rats on a progressive ratio schedule of reinforcement to investigate motivation to obtain alcohol $(301,322)$. We found that rats were slightly more motivated to work for and consume mildly more alcohol after exposure to the IA procedure as reflected by the breakpoint and intake value which was significantly higher compared to the controls.

A.

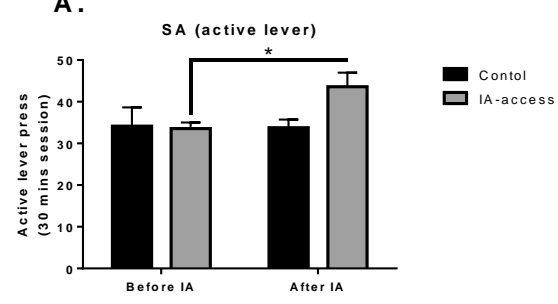

C.

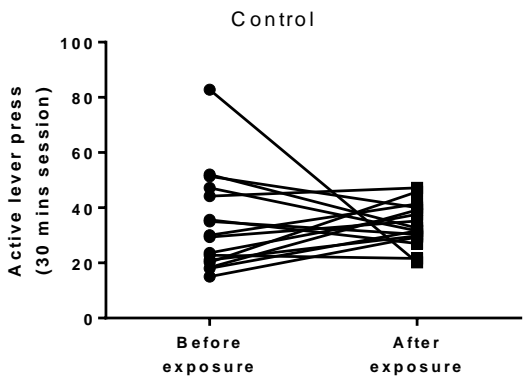

B.

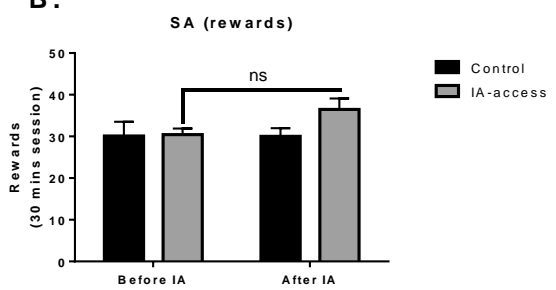

D. IA-access

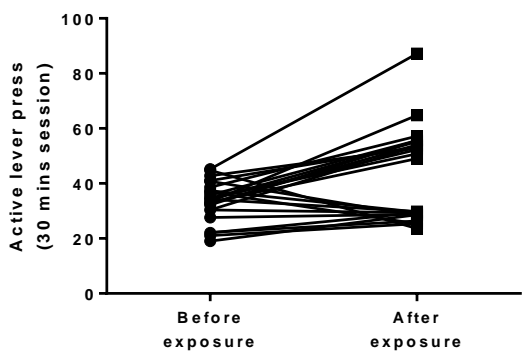

Figure 8. Effect of IA exposure on operant alcohol self-administration. IA to alcohol leads to a (mild) increase in active lever-responding (A) but not in rewards earned (B) in operant selfadministration. Animals with IA-exposure (D) but not unexposed animals (C) could be divided into escalated and non-escalated subgroups. ${ }^{*} p<0.05$ vs. control. The result are presented as mean \pm S.E.M.

\subsubsection{Intermittent access to voluntary alcohol drinking resulted in a pronounced escalation of operant alcohol self- administration in a subgroup of individuals}

Escalation of operant alcohol self-administration was operationally defined as at least a $30 \%$ increase in number of lever presses after IA compared with baseline. We observed a clear division between escalated and non-escalated subgroups within the self-administration trained group following IA. This could be due to for example the development of early physical dependence and/or a change in the perception of the positive reinforcing effects of alcohol. The intermittent pattern of alcohol-intake models repeated cycles of binge intake followed by abstinence (293). An alternative explanation is that IA with its repeated cycles of drinking and withdrawal leads to increased drinking that is driven by anticipation of a future withdrawal episode. 


\subsubsection{SR-8993 selectively suppressed alcohol-intake in individuals with escalated self-administration}

Pretreatment with SR-8993 reduced alcohol-intake in a two-bottle free choice paradigm in the group with escalated self-administration but not in the non-escalated group (Figure 9 A-F). This is supported by previous results that rats without a history of alcohol dependence are not sensitive to attenuation of alcohol self-administration by NOP or to the anxiolytic effects of NOP. With a history of dependence, administration of NOP reduces both alcohol-intake and has anxiolytic effects. A history of high alcohol-intake results in changes in NOP peptide and receptor expression (323).

A.

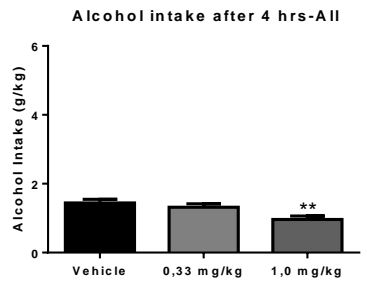

D.

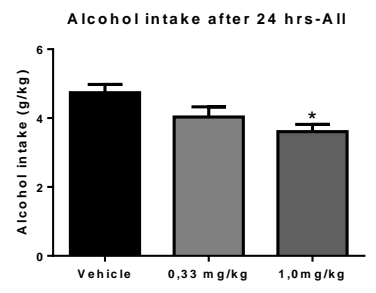

B.

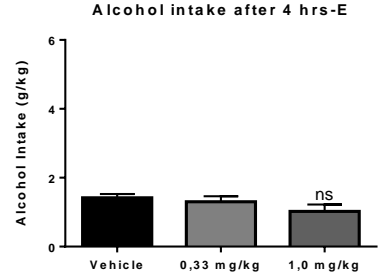

E.

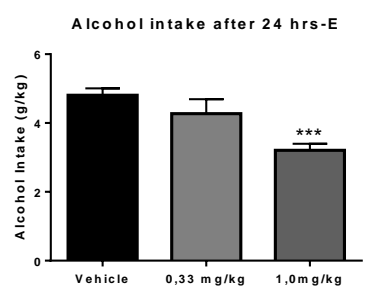

C. Alcohol intake after $4 \mathrm{hrs}-\mathrm{NE}$

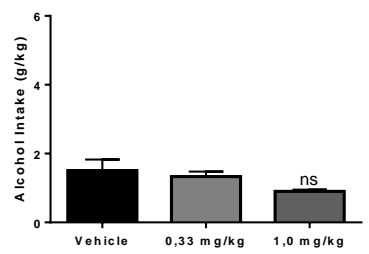

$\mathbf{F}$.

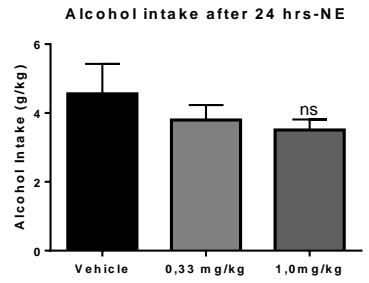

Figure 9. Escalated group of animals only showed sensitivity to treatment. Treatment response to NOP agonist SR-8993 at $1 \mathrm{mg} / \mathrm{kg}$ in voluntary alcohol-intake using a two-bottle free choice procedure. (A, D)- all animals; (B, E)- escalated animals, (C, F)- non-escalated animals. ${ }^{*} p<0.05$ vs. vehicle, ${ }^{*}$ $p<0.01$ vs. vehicle and ${ }^{\star \star *} p<0.001$ vs. vehicle. The results are presented as mean \pm S.E.M.

\subsubsection{SR-8993 potently suppressed abstinence-induced alcohol self-administration}

Pretreatment with SR-8993 dose-dependently attenuated abstinence-induced alcohol self-administration (Figure 10). Abstinence from alcohol results in a change in the reinforcement value of alcohol. AUD patients typically experience increased motivation to drink during attempts to abstain from alcohol. This is one factor that facilitates relapse to uncontrolled alcohol consumption. When relapse occurs after a period of abstinence, alcohol consumption increases compared with consumption levels before the period of abstinence (324). In animal models, alcohol-intake also increases after a period of no alcohol, which is known as the alcohol deprivation effect 
(293). Deprivation can thus be used to facilitate increased alcohol-intake in animal models of alcohol dependence (293). The effect could be due to changes in alcohol's reinforcing properties. A similar phenomenon also occurs for natural reinforcers such as palatable food and water (325). However, animal models typically do not reflect every aspect of the complex behavioral factors involved in human alcohol use. One key feature missing in animal models is the internal motivation to quit drinking during the cycles of drinking followed by abstinence.

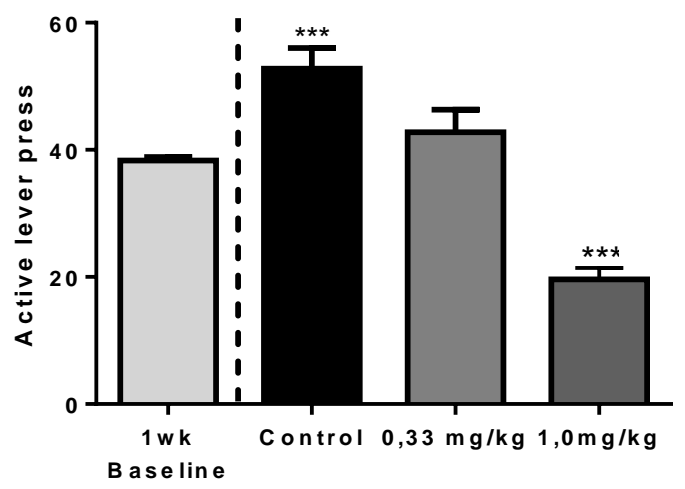

Figure 10: A three-week abstinence period significantly increased operant responding was robustly suppressed. Pretreatment with NOP agonist SR-8993 at $1 \mathrm{mg} / \mathrm{kg}$ drastically reduced operant responding to levels well below pre-abstinence baseline. ${ }^{* * *} p<0.001$ vs. 1 week baseline and ${ }^{* * *}$ $p<0.001$ vs. control. The results are presented as mean \pm S.E.M.

\subsection{The neuropeptide $\mathrm{Y}-\mathrm{Y} 2$ antagonist as a putative treatment target in alcohol use disorder: validation in rat models (Paper III)}

\subsubsection{CYM-9840 prevented acute alcohol-induced withdrawal anxiety}

CYM-9840 had no significant anxiolytic effect in the EPM when given to naïve animals but it significantly reduced acute alcohol withdrawal-induced ("hangover") anxiety observed 12 hours after alcohol administration (Figure 11). In the EPM, an anxiolytic effect in naïve animals has been observed following treatment with another NPY-Y2 receptor antagonist BIIE0246 (326) but the finding could not be repeated here. Decreased protein and gene expression of NPY and its receptors has been observed in the rat brain during alcohol withdrawal in rats $(196,327)$, strengthening the idea that the NPY system is recruited during alcohol withdrawal. 

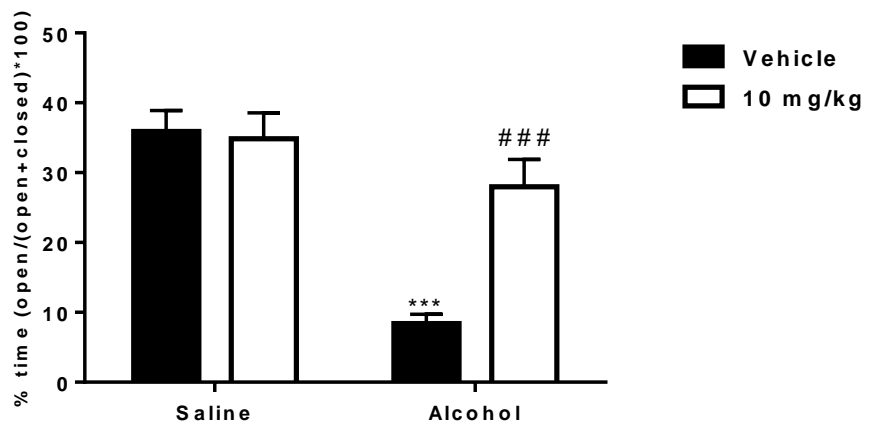

Figure 11. Pretreatment with NPY-Y2 receptor antagonist CYM-9840 attenuates withdrawalinduced anxiety on the elevated plus-maze. Pretreatment with CYM-9840 at a dose of $10 \mathrm{mg} / \mathrm{kg}$ bodyweight reversed the anxiogenic effects of an acute administration of a high dose of alcohol. Withdrawal anxiety was induced by an acute administration of alcohol (i.p; $3.0 \mathrm{~g} / \mathrm{kg}$ ) at 12 hours prior to testing using the elevated plus-maze and the pretreatment with CYM-9840 (10 mg/kg) was administered 45 mins prior to plus-maze exposure. Data presented is percent time spent on the open arm out of time spent on any arm (100\% x (open/(open+closed)) All values are given as mean \pm SEM. ${ }^{* * *} p<0.001$ vs. saline group, \#\#\# $p<0.001$ vs. vehicle-alcohol group.

\subsubsection{CYM-9840 reduced alcohol-intake in the two-bottle free choice preference drinking with 4 hour limited access}

Two-bottle free choice drinking (4 hour limited access) was significantly reduced by pretreatment with CYM-9840 (Figure 12 A-B). Our data further confirm that antagonism of central NPY-Y2 receptors attenuates alcohol drinking as previously shown that the NPY system is recruited in rats with a history of alcohol drinking (190).

A.

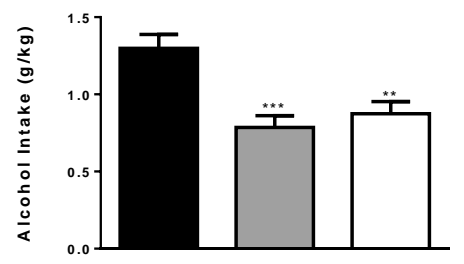

B.

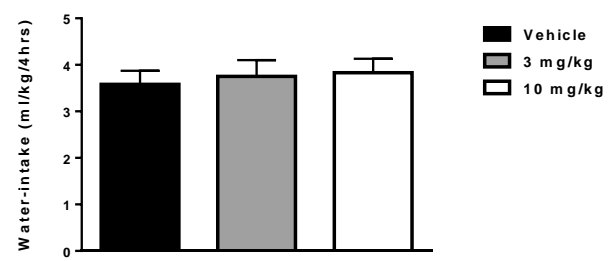

Figure 12. Effect of NPY-Y2 receptor antagonist CYM-9840 in the 4-hour limited access paradigm. Alcohol-intake (A) but not water-intake (B) in a non-operant two-bottle free choice paradigm was significantly attenuated by pretreatment with CYM-9840. Animals were given access to two bottles, one with alcohol solution and one with water, for 4 hours starting one hour into the dark phase. (A) Alcohol-intake was significantly suppressed following treatment with CYM-9840. (B) Water-intake (ml/kg bodyweight) was not affected by pretreatment with CYM-9840. All values are given as mean \pm SEM. ${ }^{* *} p<0.01$ vs. vehicle; ${ }^{* * *} p<0.001$ vs. vehicle. 


\subsubsection{CYM-9840 suppressed operant self-administration of alcohol, motivation to seek alcohol and stress- but not cue- induced reinstatement of alcohol seeking}

Pretreatment with CYM-9840 significantly suppressed operant self-administration of alcohol, progressive ratio breakpoint and stress-induced but not cue-induced reinstatement of alcohol seeking (Figure 13 A-D). The lack of effect on cue-induced reinstatement shows behavioral specificity to the stress-relieving aspect of alcohol seeking. Another NPY-Y2 receptor antagonist, BIIE0246, has been previously reported to suppress alcohol self-administration and there is evidence that this effect sensitizes in rats with a history of alcohol dependence $(189,190)$. However, this effect was not replicated with a different NPY-Y2 receptor antagonist JNJ-31020028 with systemic administration or local injection (188). Despite a lack of consensus in the literature on the role of the NPY-Y2 receptor in rat models of AUD, our results provide further support that antagonism at the central NPY-Y2 receptors could be an effective treatment strategy in AUD.

A.

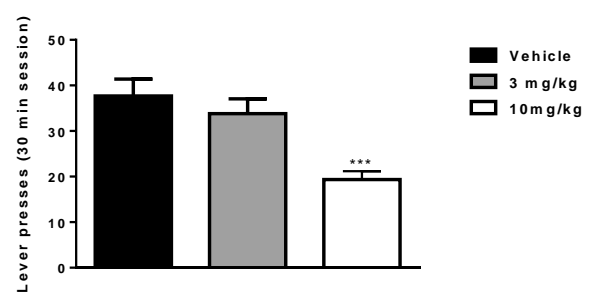

C.

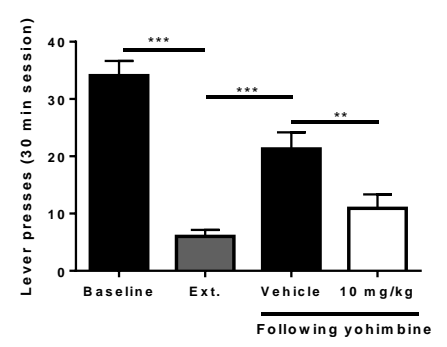

B.

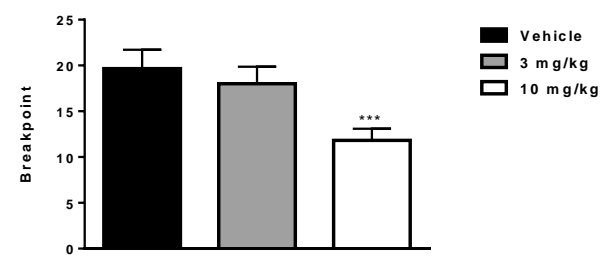

D.

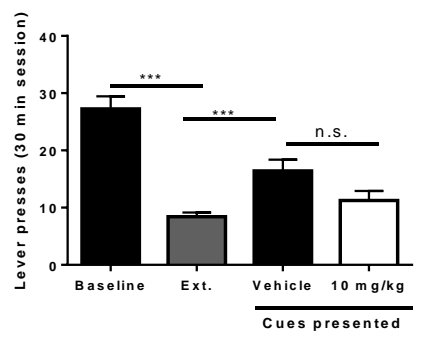

Figure 13. Effect of NPY-Y2 receptor antagonist CYM- 9840 in the alcohol self-administration, progressive ratio and relapse paradigm. CYM-9840 suppresses alcohol self-administration (A), decreases motivation to work for alcohol (B) and prevents stress- (C), but not cue- (D) induced relapselike behavior. (A) Operant self-administration of alcohol (10\% v/v) was significantly attenuated by pretreatment with 3 and $10 \mathrm{mg} / \mathrm{kg}$ CYM-9840. (B) Pretreatment with CYM-9840, significantly decreased motivation to work for alcohol delivery as measured using progressive ratio responding. Breakpoint is defined as the highest number of lever-presses the animals performed to receive one dose of alcohol 
in the drinking cup. (C) Stress-induced reinstatement by the pharmacological stressor yohimbine was blocked by pretreatment with SR-CYM-9840. (D) Exposure to alcohol-associated cues (light/orange odor) induced reinstatement of lever-pressing at the lever previously paired with alcohol delivery (extinction vs. vehicle bars). Pretreatment with CYM-9840 did not significantly affect this measure. All values are given as mean \pm SEM. ${ }^{* * \star} p<0.001$ vs. vehicle.

\subsubsection{Control experiments confirmed the behavioral specificity of the effects of CYM-9840 on alcohol-related behaviors}

A panel of control behavioral tests including locomotor activity, operant selfadministration of $0.1 \%$ saccharin, LORR, BAC, saccharin taste-preference adulteration with quinine and FST were unaffected by treatment with CYM-9840. These data reflects the behavioral and pharmacological specificity of CYM-9840 for alcohol.

\subsection{Melanin-concentrating hormone and its $\mathrm{MCH} 1$ receptor: Relationship between effects on alcohol and caloric intake (Paper IV)}

Alcohol is a unique drug in that it is both a pharmacodynamically active substance with the ability to activate brain reward systems and a source of calories. The role of $\mathrm{MCH}$ and the MCH1-R in energy balance and homeostasis is well established $(217,248$, 251). The MCH1-R antagonist GW803430 has been shown to attenuate alcohol selfadministration and block cue-induced reinstatement of alcohol seeking in naïve rats. They also reported that the effective dose of compound that was used upon several observations indicate that the action of it were behaviorally and pharmacologically specific (328). However, the effect of MCH1-R on alcohol-intake and reward in a model of escalated-intake was previously unknown. So to further investigate in this study, we extended previous findings using GW803430 by assessing the role of $\mathrm{MCH} 1-\mathrm{R}$ in regulation of caloric-intake and motivation to consume alcohol after escalated-intake.

\subsubsection{Intermittent Access to alcohol resulted in robust escalation of intake that was accompanied by a caloric shift from food to alcohol}

Food and alcohol-intake were measured during IA to $20 \% \mathrm{v} / \mathrm{v}$ alcohol. Rats were divided into groups of high and low alcohol drinkers. Rats in the high alcohol drinker group consumed over $4 \mathrm{~g} / \mathrm{kg}$ alcohol per day and had reduced food-intake on days when alcohol was available as compared with days when alcohol was unavailable. Rats in the low alcohol drinker group consumed under $2 \mathrm{~g} / \mathrm{kg}$ alcohol per day and had similar food-intake regardless of whether alcohol was available or not. Total caloricintake did not differ between groups. Regression analysis revealed a significant negative correlation between alcohol-intake and food-intake in the high alcohol drinker group. 


\subsubsection{GW803430 reduced caloric-intake from both alcohol and food in the high drinker group only}

In agreement with previous studies, we found that treatment with $\mathrm{MCH} 1-\mathrm{R}$ antagonist GW803430 resulted in a significant decrease in food-intake $(250,251,253,329)$ in both the groups, while alcohol-intake was only decreased for rats in the high alcohol drinker group (Figure 14 A-D). This indicates that $\mathrm{MCH}$ primarily regulates homeostatic caloric balance but also may also modulate alcohol reward when intake has escalated. However, alcohol is a source of calories in addition to its rewarding properties and it can be difficult to fully separate these effects. Also, because the low alcohol drinking group obtained relatively few calories from alcohol, the lack of a significant reduction of alcohol-intake with GW803430 treatment in this group may be due to a floor effect.

A.

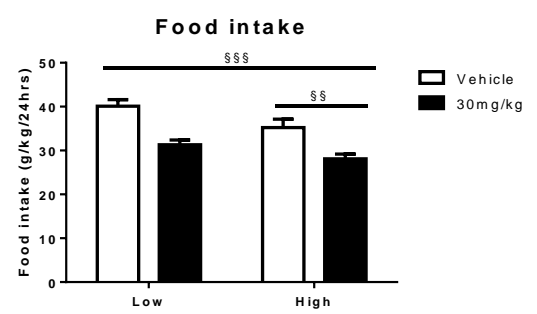

C.

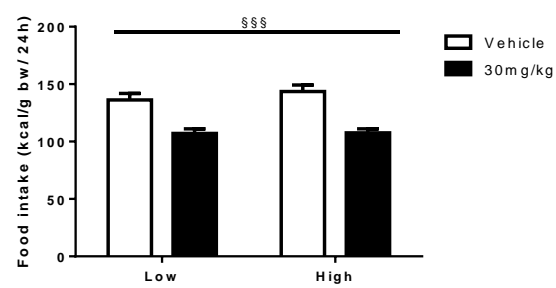

B.

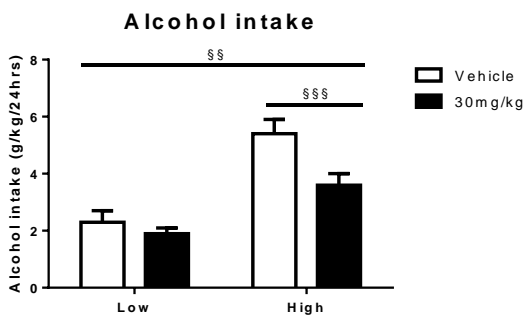

D.

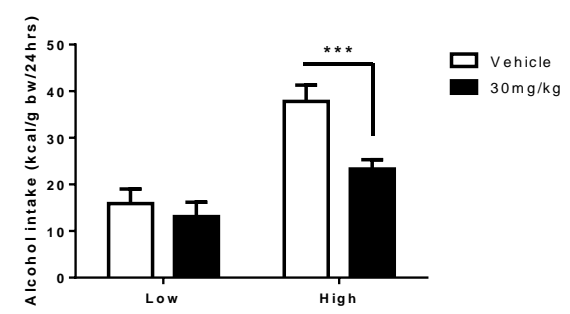

Figure 14. Food and alcohol-intake is suppressed by treatment with the melanin-concentrating hormone-1 receptor antagonist, GW803430 $(30 \mathrm{mg} / \mathrm{kg})$ in high-drinking rats. A suppression of both food (A) and alcohol-intake $\mathrm{g} / \mathrm{kg} / 24 \mathrm{~h}$ (B) following administration of GW803430 was seen in both high $(>4 \mathrm{~g} / \mathrm{kg} / 24 \mathrm{~h}$ ) and low ( $<2 \mathrm{~g} / \mathrm{kg} / 24 \mathrm{~h}$ ) drinking animals following 20 weeks of IA to $20 \% \mathrm{v} / \mathrm{v}$ alcohol. A main effect of group and treatment was noticed for both food ( $\S \S p<0.01$ and $\S \S \S p<0.001$, respectively) and alcohol-intake ( $\S \S p<0.001$ and $\S \S p<0.01$, respectively). The dose of $30 \mathrm{mg} / \mathrm{kg}$ decreased calories from food in both groups (C) shown as a main effect of group and treatment $(\S \S p<0.01$ and $\S \S \S p<0.001$, respectively) however, calories obtained from alcohol were significantly lower in high drinkers while treatment did not affect calorie intake from alcohol in low-drinking rats, ${ }^{* * *} p<0.001$, compared to vehicle, high-drinking group (D). Food and alcohol consumption was calculated as $\mathrm{kcal} / \mathrm{kg} / 24 \mathrm{~h}$. GW803430 had no impact on water-intake in either group. 


\subsubsection{Escalated alcohol self-administration induced by intermittent access was suppressed by GW803430 administration during protracted abstinence following intermittent access}

In this experiment, we further evaluated the role of $\mathrm{MCH} 1-\mathrm{R}$ in a rat model of escalated alcohol-intake. The effect of GW803430 on motivation to obtain alcohol was assessed using operant alcohol self-administration during protracted abstinence following IA. In this paradigm, GW803430 dose-dependently suppressed alcohol self-administration compared with controls (Figure $15 \mathrm{~A}$ ). While not affecting the inactive leverresponding (Figure $15 \mathrm{~B}$ ).

\subsubsection{Escalated alcohol self-administration induced by chronic alcohol vapor exposure was not suppressed by GW803430 administration during acute withdrawal}

The effect of GW803430 on motivation to obtain alcohol was assessed using operant alcohol self-administration during acute withdrawal from alcohol vapor. In contrast with results obtained during protracted abstinence from $I A$, there were no differences in alcohol self-administration during acute withdrawal between alcohol vapor exposed and air exposed control rats (Figure 15 C-D). While both methods result in persistent escalation of voluntary alcohol consumption, they offer distinct advantages. Alcohol vapor exposure induces physical dependence with the dose controlled by the experimenter, making this method well suited for assessment of effects during acute withdrawal $(330,331)$.

A.

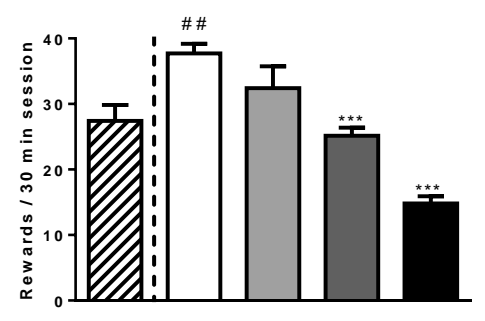

C.

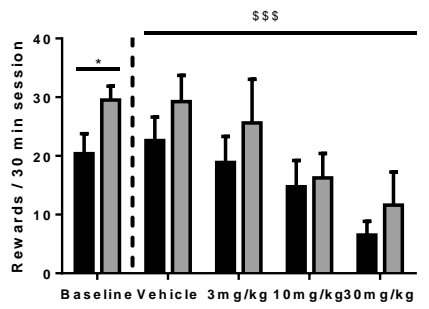

B.
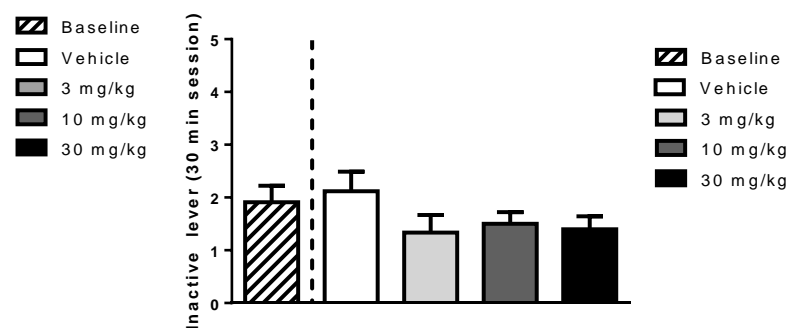

D.

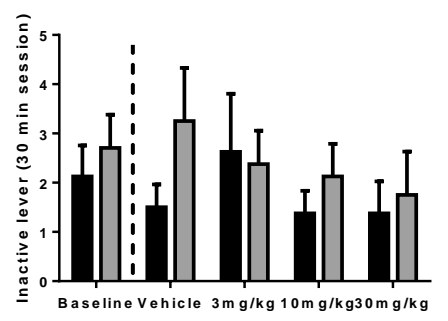


Figure 15. Pretreatment with GW803430 dose dependently attenuated lever-pressing for alcohol during protracted abstinence, but did not significantly alter lever-pressing during acute withdrawal. Treatment with the melanin-concentrating hormone-1 receptor antagonist, GW803430, attenuated lever-pressing for $20 \% \mathrm{v} / \mathrm{v}$ alcohol in animals during protracted abstinence, \#\#p $<0.01$ versus baseline; ${ }^{* \star *} p<0.001$ versus vehicle $(\mathbf{A})$. Vapor rats were self-administering significantly more during baseline, ${ }^{*} p<0.05$ versus control. A main effect of treatment was observed in acute withdrawal, $\S \S \S p<0.001$ (C). Responding at the non-alcohol paired lever was not affected by treatment in protracted abstinence (B) or acute withdrawal (D). Results are presented as average \pm SEM.

\subsubsection{Expression of $\mathrm{MCH}$ and $\mathrm{MCH} 1-\mathrm{R}$ is dysregulated in the hypothalamus and nucleus accumbens during protracted abstinence from intermittent access but not during acute withdrawal from alcohol vapor}

In this study, we demonstrated critical roles for $\mathrm{MCH}$ and $\mathrm{MCH} 1-\mathrm{R}$ in energy balance and reward modulation during protracted abstinence following IA. MCH gene expression was downregulated in the $\mathrm{LH}$, which primarily contains the cell bodies producing $\mathrm{MCH}$. $\mathrm{MCH} 1-\mathrm{R}$ gene expression was upregulated in the $\mathrm{NAc}$, which receives $\mathrm{MCH}$ projections from the $\mathrm{LH}$ (Figure $16 \mathrm{~A}-\mathrm{C}$ ). We hypothesize that these adaptations persist during protracted abstinence, which could explain the effect of GW803430 on escalated self-administration during protracted abstinence. In support of our findings, local injection of $\mathrm{MCH}$ into the NAc or the PVN increases alcohol-intake (332). This raises the possibility that alteration of the circuits that regulate both foodintake and reward underlie the escalation of alcohol consumption.

There were no changes in $\mathrm{MCH}$ or $\mathrm{MCH} 1-\mathrm{R}$ gene expression during acute withdrawal from alcohol vapor exposure. It has been previously demonstrated that repeated cycles of alcohol vapor exposure induce physical dependence and escalation of alcohol-intake in rats (330). During acute withdrawal, rats express physical withdrawal symptoms that promote alcohol-intake mainly driven by relief of these symptoms (14). Our results suggest that the $\mathrm{MCH}$ system is not involved in this process. This fits with the idea that escalation of alcohol self-administration during acute withdrawal recruits stress-mediating rather than reward-mediating pathways. 
A.

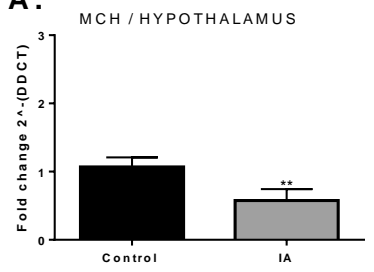

B.

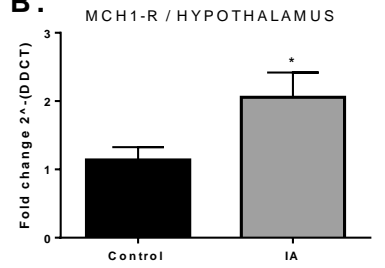

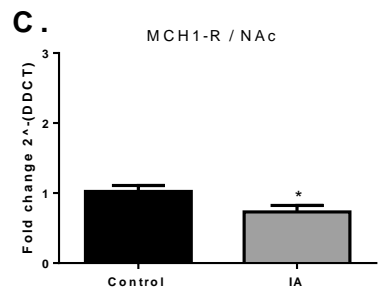

Figure 16. Changes in melanin-concentrating hormone $(\mathrm{MCH})$ and $\mathrm{MCH}-1$ receptor $(\mathrm{MCH} 1-\mathrm{R})$ mRNA expression in the hypothalamus and nucleus accumbens in protracted abstinence following IA (IA). mRNA expression for $\mathrm{MCH}$ was down-regulated while the $\mathrm{MCH} 1-\mathrm{R}$ was up-regulated in the hypothalamus during protracted abstinence following IA (A, B). Further, $\mathrm{MCH} 1-\mathrm{R}$ expression was down-regulated in the nucleus accumbens (NAc) compared to control rats (C). ${ }^{* *} p<0.01$ versus control, ${ }^{*} p<0.05$ versus control and ${ }^{*} p<0.05$ versus control. Results are presented as average \pm SEM. 


\section{SUMMARY}

Various central neuropeptides have been implicated in the regulation of anxiety, stress reward and alcohol-intake. This thesis focused on the role of three of these; NOP, NPY and $\mathrm{MCH}$ and their receptors in the pathophysiology of AUD. Modern chemical synthesis technology makes it possible to develop small non-peptide molecules targeting specific receptor systems in the CNS. We have used this strategy of modulating the activity of these receptors using pharmacological interventions designed as specific agonists or antagonists in order to evaluate behavioral outcomes in animal models of AUD.

First, NOP which is widely expressed in the CNS has been implicated in a wide range of central effects including drug abuse. Activation of the NOP receptor has been suggested to play critical roles in alcohol reward and in compulsive alcohol-seeking behavior. The differential effects of NOP receptor activation have been hypothesized to be due to differential activation of intracellular signaling pathways following ligand binding or biased agonism. The recent development of novel, small molecule agonists for the NOP receptor that can easily cross the blood brain barrier gives us a unique possibility to further evaluate this receptor as a novel pharmacological treatment target for AUD.

We found that stimulation of the NOP receptor using the novel, small molecule and peripherally available agonist SR-8993 reversed acute alcohol withdrawal-induced "hangover" anxiety. It also dose-dependently resulted in decreased operant SA and decreased voluntary two-bottle free choice drinking of alcohol. In addition, it blocked both cue- and stress-induced reinstatement of alcohol seeking, models for relapselike behavior. SR-8993 also attenuated escalated consumption in the IA model and suppressed operant SA in rats with a history of escalation. It was highly selective for the NOP receptor and the specificity of its effects on alcohol-related behavior was supported by our findings that it did not affect behavior in control experiments measuring locomotor activity, $0.1 \%$ saccharin $\mathrm{SA}$, saccharin/quinine taste adulteration, water-intake, FST, LORR and BAC.

The progression to a diagnosis of AUD includes increased craving for and escalation of alcohol consumption over time accompanied by repeated episodes of abstinence and relapse. We modelled this with a combination of operant SA and IA to alcohol. We found that a previous history of operant SA resulted in increased intake of and preference for alcohol during IA. Additionally, IA exposure resulted in slightly increased alcohol SA and higher PR breakpoints. Pretreatment with SR-8993 attenuated alcoholintake during IA and suppressed abstinence-induced operant SA of alcohol.

Taking all of these results together, NOP receptor agonist SR-8993 had a broad range of in-vivo activity and the NOP receptor represents a viable treatment target for AUD. We hypothesize that it works by modulating or inhibiting the positive reinforcing properties of alcohol. 
Second, NPY is widely distributed in the brain and highly expressed in brain regions modulating emotion, anxiety and motivation. NPY is orexigenic and anxiolytic and has been implicated in playing key roles in the regulation of alcohol consumption, withdrawal-induced anxiety and stress-induced reinstatement of alcohol seeking in animal models and potentially in AUD in humans.

We evaluated CYM-9840 a novel, small molecule NPY-Y2 receptor antagonist that penetrates the blood brain barrier in animal models of alcohol-related behaviors. We found that pretreatment with CYM-9840 reversed acute alcohol withdrawal-induced "hangover" anxiety, reduced alcohol-intake in two-bottle free choice preference drinking, suppressed alcohol SA and lowered the PR breakpoint reflecting decreased motivation to work for alcohol. It also significantly blocked stress-induced but not cueinduced reinstatement of alcohol seeking. These effects were specific to alcohol related behaviors, as CYM-9840 did not affect the control behaviors we measured. Thus, antagonism of NPY-Y2 receptor can be a possible effective treatment strategy, particularly for the anxiety- and stress-related mechanisms that are difficult to treat in the later stages of AUD.

Third, a growing body of evidence suggests that $\mathrm{MCH}$, a hypothalamic neuropeptide involved in the regulation of energy homeostasis and plays a role in AUD. $\mathrm{MCH}$ peptides act on the MCH1-R in rodents. As $\mathrm{MCH}$ is implicated in the regulation of foodintake and energy homeostasis it could also be a valuable target for treatment of obesity (253). Further, it modulates stress- and anxiety-related behaviors. MCH1-R has not been previously evaluated for its role in long-term exposure to alcohol and escalated alcohol consumption. Thus, we assessed the role of MCH1-R in the regulation of caloric-intake and motivation to consume alcohol during high and escalated alcohol-intake state using MCH1-R antagonist GW803430.

Here our findings demonstrated a role of $\mathrm{MCH}$ and $\mathrm{MCH} 1-\mathrm{R}$ in modulation of alcoholintake, most likely through intracellular mechanisms involving both caloric consumption and motivation to obtain reward. We found that rats trained with IA to drink high amounts of and show more preference for alcohol showed compensatory reduced food-intake when obtaining more calories from alcohol. Treatment with GW803430 suppressed both food and alcohol consumption together. Alcohol SA was strongly attenuated following treatment with GW803430 during protracted abstinence after IA drinking and this was correlated with downregulation of $\mathrm{MCH}$ peptide gene expression and upregulation of $\mathrm{MCH} 1-\mathrm{R}$ gene expression in the hypothalamus. Although there were no changes in the gene expression during acute withdrawal from alcohol vapor-induced dependence and treatment with GW803430 didn't resulted in an attenuated responding for alcohol during acute withdrawal period which is likely and predominantly driven by stress-related mechanisms.

Taking all of these results together, we found that central neuropeptides are attractive as potential treatment targets for anxiety and for AUD. The neuropeptides NOP, NPY and $\mathrm{MCH}$ are involved in anxiety and in AUD. Manipulating the activity of their 
receptors with non-peptidergic small molecule agonists or antagonists approach is a useful way to evaluate these neuropeptides as possible therapeutic targets to treat anxiety disorders and AUD. We believe that these findings could put the foundation and pave the way for other upcoming future compounds targeting the NOP, NPY and $\mathrm{MCH}$ receptor system with better activity profile in terms of clinical safety and tolerability. 


\section{CONCLUDING REMARKS}

Increased knowledge of the neurobiological processes driving the underlying biological mechanisms of AUD is a prerequisite in the search for novel medications to treat this disease. Three essential sources can contribute information and support development of new medications. The first is basic science research focusing on the neurobiological phenomena and underlying mechanisms in different stages of the addiction cycle. The second is research on effects of currently approved or potential treatments in animal models of the different stages of addiction. The third is clinical research on the currently approved or potential treatments in different patient populations. Data from these three research areas will lead to increasingly complete knowledge of neurotransmitter and neuropeptide systems modulating different aspects of the alcohol dependence such as reward-driven binge intoxication, negative and aversive withdrawal symptoms and the craving that leads to relapse. The more we know about the mechanisms behind different aspects of addiction, the better equipped we will be to treat individual patients effectively.

AUD contributes significantly to the global burden of disease in terms of financial costs, morbidity and mortality. Mild symptoms can resolve without treatment but more severe symptoms are often underdiagnosed and undertreated. Diagnosis and treatment strategies need improvement. The most effective treatment for a given individual patient could be behavioral, pharmacological or a combination of the two and this needs to be based on practical rather than theoretical considerations. Patients who initially reject abstinence as a treatment target could be offered treatment aimed at reduction of alcohol-intake for example.

There are many unresolved questions in the field of alcohol research. Preclinical studies and early phase clinical trials have focused on a wide variety of potential new treatments for AUD such as targeting neuropeptide, opioid, cannabinoid, nicotinic, dopaminergic, glycinergic, GABAergic and glutamatergic receptors. A few possible new treatments for AUD based on recent research include gabapentin, topiramate, baclofen and varenicline $(4,54)$. The most relevant neurotransmitter systems and thus the appropriate treatment will likely be based on individual differences between patients as well as the target phase of the addiction cycle.

Central neuropeptide systems that are involved in the regulation of mood, anxiety, depression and reward offer attractive possible treatment targets to add to the currently available treatments. Using a combination of preclinical animal models, molecular techniques and clinical trials to identify targets and validate candidate treatments is the way forward in finding the most effective treatments for AUD. 


\section{FUTURE PERSPECTIVES}

GPCRs targeted drug discovery has recently focused on ligand bias where a ligand selectively engages one signaling pathway while avoiding or inactivating others to produce a novel pharmacological profile. The NOP receptor is a GPCR coupling to beta-arrestin 2/3 in addition to the G-protein $(107,108)$. Beta-arrestin 2/3 has been shown to regulate G-protein signaling and to be involved in the initiation of $\mathrm{G}$-protein desensitization, internalization and independent signal transduction (333). The distinct effects of different NOP agonists on reward may be due to differences in subsequent intracellular mechanisms following binding of the agonist to the receptor and betaarrestin $2 / 3$ could play a critical role. Additionally, the intracellular pathways regulated by beta-arrestin and G-protein pathways differ. The activation of the NOP receptor with subsequent G-protein recruitment leads to activation of ERK (334).

Thus, this present preliminary work aimed to evaluate the effect of the NOP receptor agonist SR-8993 on reward-related behavior as measured by CPP in C57BL/6 wildtype and beta-arrestin 2/3 knockout mice. Furthermore, phosphorylation of ERK induced by systemic alcohol administration and the role of SR-8993 in blocking the phosphorylation was measured in C57BL/6 wild-type mice by IHC. 


\subsection{SR-8993 blocked expression of alcohol-induced CPP both at a dose of 1 and $3 \mathrm{mg} / \mathrm{kg}$}
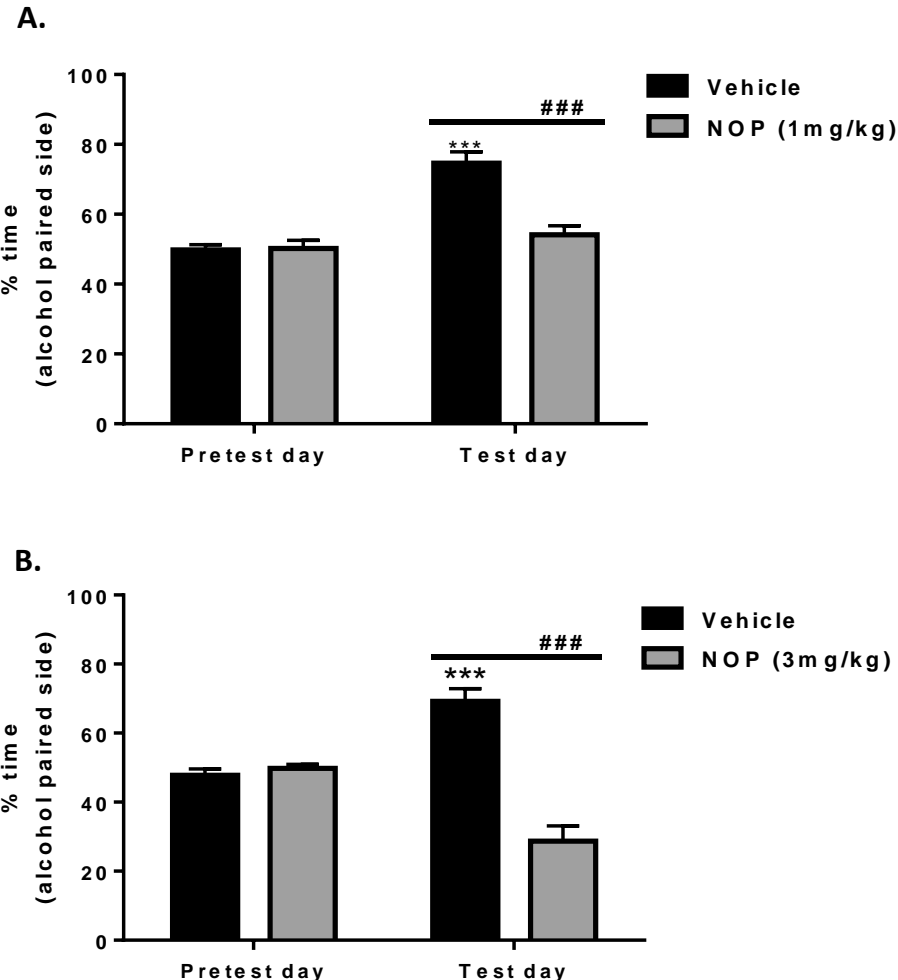

Figure 17. Pretreatment with SR-8993 blocked expression of alcohol-induced CPP $(2 \mathrm{~g} / \mathrm{kg})$ in C57BL/6 mice. Alcohol at a dose of $2 \mathrm{~g} / \mathrm{kg}$ robustly induced conditioned place preference using an unbiased design which was blocked by SR-8993 at a dose of 1 and $3 \mathrm{mg} / \mathrm{kg}$. The data is represented as \% time spent in the alcohol paired side in the pretest and test day. (A) On the test day the vehicletreated animal spent significantly more time in the alcohol-paired side compared to the pretest (\#\#\#p<0.001) confirming the establishment of alcohol CPP and more time than NOP-treated animal spent on the alcohol-paired side compared to the test $\left({ }^{\star \star *} p<0.001\right)$. (B) On the test day, the vehicletreated animal spent significantly more time in the alcohol-paired side compared to the pretest (\#\#\#p<0.0002) confirming the establishment of alcohol CPP and more time than NOP-treated animal spent on the alcohol-paired side compared to the test $\left({ }^{* * *} p<0.0001\right)$. The results are presented as mean \pm S.E.M. 
7.2 SR-8993 blocked alcohol-induced CPP via a beta-arrestin independent mechanism

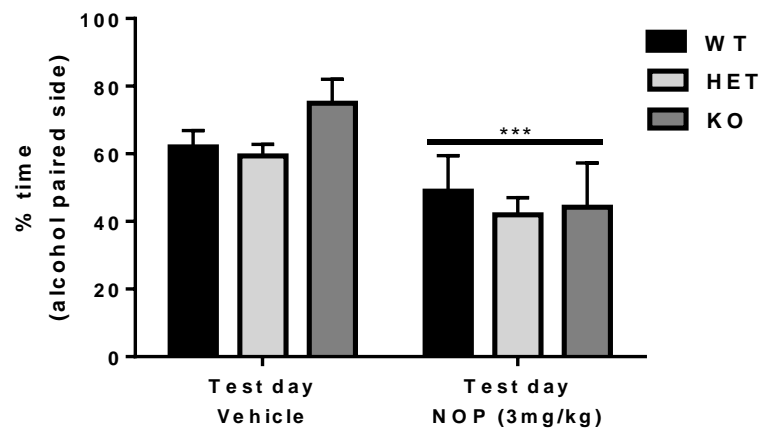

Figure 18. SR-8993 (3 mg/kg) significantly reduced expression of alcohol-induced CPP regardless of genotype. Alcohol at a dose of $2 \mathrm{~g} / \mathrm{kg}$ robustly induced conditioned place preference in beta-arrestin knock-out mice using an unbiased design which was blocked by SR-8993 at a dose of 3 $\mathrm{mg} / \mathrm{kg}$. The data is represented as \% time spent in the alcohol paired side in the pretest and test day. On the test day, the NOP-treated animal spent significantly less time in the alcohol-paired side compared to the vehicle-treated animals $\left({ }^{* *} p<0.0001\right)$. Data are presented as mean \pm S.E.M. 


\subsection{Pretreatment with SR-8993 attenuates acute alcohol-induced phosphorylation of ERK in key brain regions}

A.

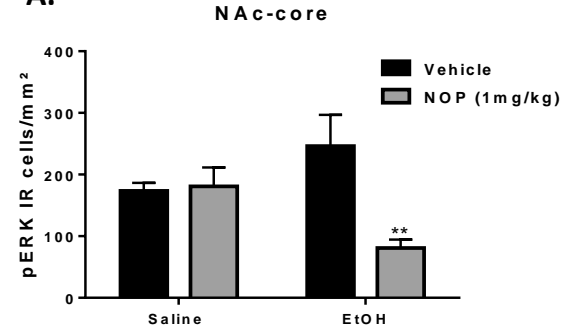

C.
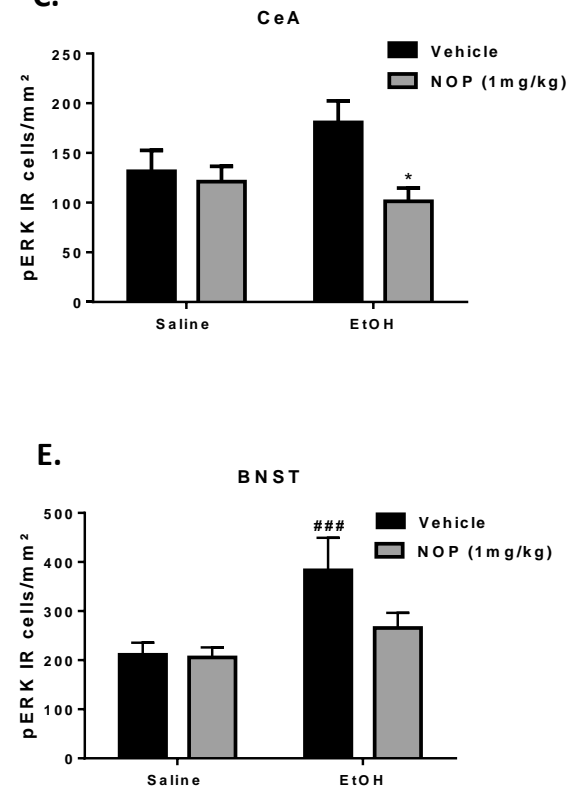

B.

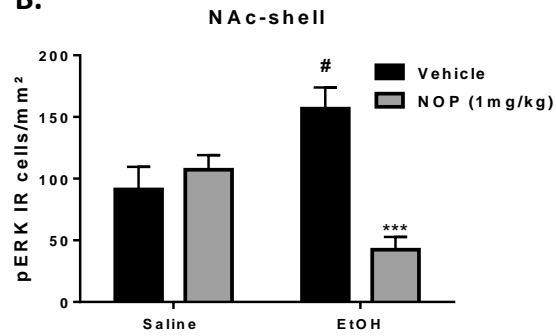

D.

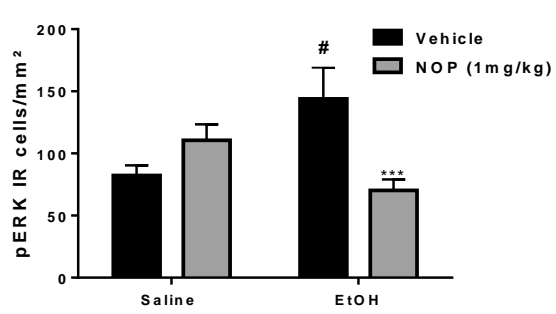

Figure 19. Acute alcohol $(2 \mathrm{~g} / \mathrm{kg})$ induced ERK phosphorylation in C57BL6 mice was blocked by pretreatment with NOP agonist (1 $\mathrm{mg} / \mathrm{kg}$ ) in different brain regions (NAC-core, NAC-shell, CeA, BMA and BNST). (A). Mice injected with an acute dose of alcohol at $2 \mathrm{~g} / \mathrm{kg}$ displayed a higher level of ERK activation in the NAc-core which was blocked by pretreatment with SR-8993 compared to the vehicle-alcohol group ( $\left.{ }^{* *} \mathrm{p}<0.002\right)$. (B). Alcohol induces a robust level of ERK activation in the NAc-shell $(\# p<0.01)$ which was potently blocked by pretreatment with SR-8993 compared to the vehicle-alcohol group $\left({ }^{* * *} \mathrm{p}<0.0002\right)$. (C). There is a trend in levels of $\mathrm{p}$-ERK activation in the $\mathrm{CeA}$ after alcohol injection compared to the vehicle-saline group which was blocked by pretreatment with $S R-8993\left({ }^{*} p<0.02\right)$. (D). Alcohol at $2 \mathrm{~g} / \mathrm{kg}$ induced a rapid activation of ERK in the BMA compared to the vehicle-saline group 
$(\# p<0.01)$ which was completely abolished with NOP pretreatment compared to the vehicle-alcohol group $\left({ }^{* *} p<0.004\right)$. (E). Alcohol induces a rapid activation of ERK in the BNST compared to the vehiclesaline group (\#\#\#p<0.006). However, a trend was noticed for NOP pretreatment $(p=0.09)$ compared to vehicle-saline group. Data represent the mean number of $p$-ERK immunoreactive cells per square millimeter. Left and right side of the regions were counted. The results are presented as mean \pm S.E.M.

In summary, our preliminary data demonstrated the role of NOP receptor activation by using selective and potent NOP receptor agonist SR-8993 which attenuates the rewarding effects of alcohol (as evaluated using the CPP paradigm) and blocks alcohol-induced ERK phosphorylation (as evaluated using $\mathrm{IHC}$ ) in different brain areas that are associated with drug reward via a beta-arrestin independent mechanism. Further investigation is on-going for this project. 


\section{ACKNOWLEDGEMENT}

These four years of Ph.D study at the Faculty of Health Sciences, Linköping University, Sweden means really a lot to me. It was a time of gaining vast knowledge and experience, also a period of self-improvement and self-discipline. I am so happy to have the opportunity to do and complete this. Without so many encouragement and support these projects and thesis work could not have been completed. I would like to express my thanks and gratitude to all of those who made all these projects possible, for being a real support at work and everywhere else in my life. I have enjoyed each and every aspect of it a lot.

I would also like to recall the pity \& kindness of Almighty Allah \& thank Him for showing me the right path of securing special knowledge in science.

First and foremost I would like to express my sincere thanks and gratitude to my supervisor Dr Annika Thorsell for her valuable idea, support, guidance \& supervision during the tenure of my master's degree project as well during my Ph.D studies which has enabled me to reach this end. Thank you for giving me this opportunity in life to develop and gain experience in the field of addiction neuroscience!

My co-supervisor Dr David Engblom for his endless support and being so active \& helpful always. I wish to use your numerous knock-out mouse lines in the near future!

Dr Markus Heilig for being an ideal mentor and a man with great scientific ideas \& values. Thanks for keeping your door open for us and for always supporting us no matter what it is. I wish I could spend more time under your outstanding guidance \& competent supervision!

Thanks to all my co-authors Wahlestedt C, Brothers S, Sartor G, Holm L, Roberts E, Mittapalli G, Cameron M, Karlsson C, Rehman F, Pitcairn C, Barbier E, Barchiesi R, Hansen M, Steensland P \& Gehlert D. This thesis works would have been impossible without my valuable \& wonderful co-authors.

I am grateful to Dr Camilla Karlsson, Dr Alison Atkins, Dr Estelle Barbier, Dr Eric Augier, Dr Daniel Nätt, Dr Esi Domi, Dr Li Xu, Gaëlle Augier, Riccardo Bachiesi, Anna Asratian, Dr Lovisa Holm, Dr Kalle Björk, Dr Susanne Hilke, Dr Michael Fritz, Anna Klawonn, Dr Maarit Jaarola, Dr Andrea Stojakovic, Dr Anand Singh, Asia Zajdel, Elahe Mirrasekhian, Dr Daniel Willhem, Dr Anna Nilson, Dr Sarah Lindström, Dr Gonzalo Manuel, Dr Unn Kugelberg \& Dr Kiseko Shionoya for your kind support, help, advice, technical and laboratory support \& regular guidance throughout the period of my time in the lab. I would like to express my hard core thanks to all our great \& hard-working past and present project students of the lab for their help, care, sharing, knowledge, ideas, constructive criticism and discussions. Special thanks to Amanda Ljung \& Emelie Krantz for the collaboration with the high fat diet project. Thanks for all the coffees, fikas, happy-hours, dinners, julbord, lab-retreats, courses and conferences together :-)

Thank you Miruna Gavrila (Mia) for the crash course in Microsoft word and for all the valuable tips \& helps.

Dr Alison Atkins for collaborating with me to count the cells from the IHC experiments and taking your time to proof-reading this thesis work for English and grammar. 
Dr Shadi Jafari for the help to sketch the cover picture of this Ph.D thesis book. You are great! Hope you are having a good time in New-York.

I would like to extend my sincere thanks to Dr Susana Cristobal, Dr Anders Rosén, Dr Anders Blomqvist, Dr Peter Strålfors, Dr Mats Söderström, Dr Anita Öst, Dr Mattias Alenius and all the members of their research group for always being so friendly and helpful, for the fikas and laughs around the coffee-table, julbord, cellbiology parties \& IKE day celebration.

I would also like to express my special thanks to the core facility unit, research members and employees of IKE, Cell-biology \& KEF building for their help, care, support \& cooperation.

Lotta J, Ulla B, Anna W, Camilla H, Annette K, Eva Å, Pernilla E, Martina K, Helena J, Erik M \& Emad $\mathbf{S}$ for all the great administrative, logistics and technical supports during my time at IKE.

I want to extend my sincere thanks to all the past and present staffs of the animal facility at CBR1, CBR2 \& Annexet for their care, support and for being so friendly \& helpful. My sincere thanks to Anders, Linda, Nettan, Emma, Björn, Petra, Anna, Monika, Anne, Marie, Andrea, Jonas, Albin, Cissi, Alexandra, Rebecca, Klara, Emelie L \& Emelie J.

I would like to take the opportunity to thank Dr Annelie Lindström \& Gunilla Linghammar who kept an eye on my progress during the time as a Ph.D student and for extended your cooperation when I needed the most help, advice and guidance.

My hearty thanks to Dr Jonas Wetterö for being always so helpful and extending all the supports during my master's studies period. YOU ARE A GREAT MENTOR!

Dr Katarina Kågedal \& Dr Camilla Karlsson for taking your time writing me the support letter to socialstyrelsen and helping me out the most when needed.

Dr Camilla, Dr Katarina, Dr Adena \& Dr Masuda for always encouraging and helping me to start practising as a licensed pharmacist in Sweden. God bless you guys!

Thank you Emelie Krantz, Elahe Mirrasekhian, Dr Andreas Lewander, Anders Delleskog \& Linda Bergström for helping me to buy an apartment here at Linköping. Edgars Butanovs for assembling all the furnitures, etc for me.

Arif Ahmed- my partner in all the crimes. Thanks for all the helps with experiments and cookings. I will never forget our hectic experiments running so many animals together and the crazy weekend bottle refilling \& weighing time. Good luck for your future! YOU ARE GREAT :)

My photographer friends Avijit, Jinto \& Rupu for capturing all my amazing pictures \& making my time in Linköping so vivid, colourful and memorable. SPLENDID JOB :)

Thank you Sharna, Imran, Saidee and Udoy for picking up from Stockholm Arlanda airport upon my first arrival to Sweden. Afzal and Dalil uncle, Ratna and Lasmin aunty for being so caring, loving and encouraging. Menen, Peea and Atif for being so loving, caring and a great host. 
Mazed mama, Lucky mami, Rony bhaiya, Boudi aunty, Shefali aunty, Nazrul aunty, Zaman teacher and Sirjee for your help, advice, support, love and care.

Risul Amin for being such a great brother, mentor and so supportive from the first day of my university life back in Bangladesh and following to Sweden. Thanks for sharing all your valuable ideas, tips and guiding me throughout. GOD BLESS YOU!

Dr Miraz, Dr Atik, Dr Mayur, Sandeep, Kamol, Rima, Shanta, Daniel, Shumon, Saikat \& Sanjeeb thank you for all the advices, helps, tips and ideas in your busy schedule. THANKS FOR ALL THE GREAT COOKING SESSIONS!

My past and present flat-mates, Shahin, Kona, Jely, Rahul \& Juliana for all the supports, helps, cleanings, cookings and for bearing all my crazy jokes \& complains.

My heartfelt thanks to all the great and active members of Bangladesh society at Linköping, TT11 and local-bus for all the funs, gossips, trip to Öland, Eid parties \& celebrations, get-togethers \& memories!

Thanks to the International Student Association for organizing successful events like International Day, Systems Neurobiology for arranging Neuroretreats \& DOMFIL for the annual HURS \& banquets; and for the all poster \& talk sessions, awards, prizes \& recognitions!

ISA, FUN, ERAB, DOMFIL \& Systems Neurobiology for the trips, travel awards \& prizes!

My wholehearted gratitude and respect also goes to my beloved father Abdul Aziz \& mother Asma Aziz for all sorts of help, advice, support and unconditional love. Thanks for being so wonderful parents and for all the inspirations, blessings, gifts and parcels from Bangladesh :)

I am very grateful to my loving sister Anika Aziz (Khushbu), brother-in-law Raihan Nasir Bhuiyan, little Wahiya (Ontu) and all my friends \& well-wishers at home and abroad, who has supported and encouraged me enormously and all of them who caused even a little to my effort as well-wishers. May Almighty Allah bless and help us all to render something for the cause of the mankind.

And of course last but not the least, thanks to all the rats and mice that made all the projects \& the results for this Ph.D thesis possible! 


\section{LIST OF REFERENCES}

1. Connor JP, Haber PS, Hall WD. Alcohol use disorders. Lancet. 2016;387(10022):988-98.

2. $\quad$ Dervaux A, Laqueille X. Alcohol use disorders. Lancet. 2016;387(10035):2292.

3. Barnett R. Alcohol use disorders. Lancet. 2017;389(10064):25.

4. Connor JP, Haber PS, Hall WD. Alcohol use disorders. The Lancet. 2016;387(10022):988-98.

5. Organization WH, Unit WHOMoSA. Global status report on alcohol and health, 2014: World Health Organization; 2014.

6. Franck J, Jayaram-Lindstrom N. Pharmacotherapy for alcohol dependence: status of current treatments. Curr Opin Neurobiol. 2013;23(4):692-9.

7. Brown SA, Goldman MS, Inn A, Anderson LR. Expectations of reinforcement from alcohol: their domain and relation to drinking patterns. J Consult Clin Psychol. 1980;48(4):419-26.

8. Association AP. Diagnostic and statistical manual of mental disorders (DSM5®): American Psychiatric Pub; 2013.

9. Association D-AP. Diagnostic and statistical manual of mental disorders. Arlington: American Psychiatric Publishing. 2013.

10. Koob GF, Volkow ND. Neurobiology of addiction: a neurocircuitry analysis. Lancet Psychiatry. 2016;3(8):760-73.

11. Donovan DM, Marlatt GA. Assessment of expectancies and behaviors associated with alcohol consumption. A cognitive--behavioral approach. J Stud Alcohol. 1980;41(11):1153-85.

12. Grusser SM, Wrase J, Klein S, Hermann D, Smolka MN, Ruf M, et al. Cueinduced activation of the striatum and medial prefrontal cortex is associated with subsequent relapse in abstinent alcoholics. Psychopharmacology (Berl). 2004;175(3):296-302.

13. Sinha R, Li CS. Imaging stress- and cue-induced drug and alcohol craving: association with relapse and clinical implications. Drug Alcohol Rev. 2007;26(1):25-31.

14. Heilig M, Koob GF. A key role for corticotropin-releasing factor in alcohol dependence. Trends in neurosciences. 2007;30(8):399-406.

15. Mayo-Smith MF, Beecher LH, Fischer TL, Gorelick DA, Guillaume JL, Hill A, et al. Management of alcohol withdrawal delirium. An evidence-based practice guideline. Arch Intern Med. 2004;164(13):1405-12.

16. Heilig M, Egli M, Crabbe JC, Becker HC. Acute withdrawal, protracted abstinence and negative affect in alcoholism: are they linked? Addict Biol. 2010;15(2):169-84. 17. Heilig M, Goldman D, Berrettini W, O'Brien CP. Pharmacogenetic approaches to the treatment of alcohol addiction. Nat Rev Neurosci. 2011;12(11):670-84.

18. Koob GF, Le Moal M. Plasticity of reward neurocircuitry and the 'dark side' of drug addiction. Nat Neurosci. 2005;8(11):1442-4.

19. Piko B. Perceived social support from parents and peers: which is the stronger predictor of adolescent substance use? Substance use \& misuse. 2000;35(4):617-30.

20. Kendler K, Gardner C, Dick D. Predicting alcohol consumption in adolescence from alcohol-specific and general externalizing genetic risk factors, key environmental exposures and their interaction. Psychological medicine. 2011;41(7):1507-16.

21. Zucker RA. The developmental behavior genetics of drug involvement: overview and comments. Behavior Genetics. 2006;36(4):616-25.

22. ENOCH MA. Genetic and environmental influences on the development of alcoholism. Annals of the New York Academy of Sciences. 2006;1094(1):193-201. 
Heath AC, Whitfield JB, Martin NG, Pergadia ML, Goate AM, Lind PA, et al. A quantitative-trait genome-wide association study of alcoholism risk in the community: findings and implications. Biol Psychiatry. 2011;70(6):513-8.

24. Baumeister RF, Tice DM. Point-counterpoints: Anxiety and social exclusion. Journal of social and clinical Psychology. 1990;9(2):165-95.

25. Kushner MG, Sher KJ, Beitman BD. The relation between alcohol problems and the anxiety disorders. The American Journal of Psychiatry. 1990;147(6):685.

26. Kessler RC, McGonagle KA, Zhao S, Nelson CB, Hughes M, Eshleman S, et al. Lifetime and 12-month prevalence of DSM-III-R psychiatric disorders in the United States: results from the National Comorbidity Survey. Archives of general psychiatry. 1994;51(1):819.

27. Morris EP, Stewart SH, Ham LS. The relationship between social anxiety disorder and alcohol use disorders: a critical review. Clin Psychol Rev. 2005;25(6):734-60.

28. Pogue YZ, Hakes JK, Sloan FA. Is Major Depression Linked to AlcoholImpaired Driving? Subst Use Misuse. 2017:1-12.

29. Gilman SE, Abraham HD. A longitudinal study of the order of onset of alcohol dependence and major depression. Drug Alcohol Depend. 2001;63(3):277-86.

30. Grant BF, Harford TC. Comorbidity between DSM-IV alcohol use disorders and major depression: results of a national survey. Drug Alcohol Depend. 1995;39(3):197-206.

31. King KM, Karyadi KA, Luk JW, Patock-Peckham JA. Dispositions to rash action moderate the associations between concurrent drinking, depressive symptoms, and alcohol problems during emerging adulthood. Psychology of addictive behaviors. 2011;25(3):446.

32. Spanagel R. Alcoholism: a systems approach from molecular physiology to addictive behavior. Physiological reviews. 2009;89(2):649-705.

33. Kendler KS, Gardner CO, Prescott CA. Toward a comprehensive developmental model for alcohol use disorders in men. Twin Res Hum Genet. 2011;14(1):1-15.

34. Nestler EJ. Is there a common molecular pathway for addiction? Nat Neurosci. 2005;8(11):1445-9.

35. Spanagel R, Weiss F. The dopamine hypothesis of reward: past and current status. Trends in neurosciences. 1999;22(11):521-7.

36. Di Chiara G, Bassareo V, Fenu S, De Luca MA, Spina L, Cadoni C, et al. Dopamine and drug addiction: the nucleus accumbens shell connection. Neuropharmacology. 2004;47 Suppl 1:227-41.

37. Koob GF, Volkow ND. Neurocircuitry of addiction. Neuropsychopharmacology. 2010;35(1):217-38.

38. Koob GF. Focus on: Neuroscience and treatment: the potential of neuroscience to inform treatment. Alcohol Res Health. 2010;33(1-2):144-51.

39. Costardi JV, Nampo RA, Silva GL, Ribeiro MA, Stella HJ, Stella MB, et al. A review on alcohol: from the central action mechanism to chemical dependency. Rev Assoc Med Bras (1992). 2015;61(4):381-7.

40. Kitson TM. The disulfiram--ethanol reaction: a review. J Stud Alcohol. 1977;38(1):96-113.

41. Peachey JE, Naranjo CA. The use of disulfiram and other alcohol-sensitizing drugs in the treatment of alcoholism. Research advances in alcohol and drug problems: Springer; 1983. p. 397-431.

42. Jorgensen $\mathrm{CH}$, Pedersen B, Tonnesen H. The efficacy of disulfiram for the treatment of alcohol use disorder. Alcohol Clin Exp Res. 2011;35(10):1749-58.

43. O'Malley SS, Jaffe AJ, Rode S, Rounsaville BJ. Experience of a "slip" among alcoholics treated with naltrexone or placebo. Am J Psychiatry. 1996;153(2):281-3. 
44.

Volpicelli JR, Watson NT, King AC, Sherman CE, O'Brien CP. Effect of naltrexone on alcohol "high" in alcoholics. Am J Psychiatry. 1995;152(4):613-5.

45. Garbutt JC, Greenblatt AM, West SL, Morgan LC, Kampov-Polevoy A, Jordan HS, et al. Clinical and biological moderators of response to naltrexone in alcohol dependence: a systematic review of the evidence. Addiction. 2014;109(8):1274-84.

46. Paille FM, Guelfi JD, Perkins AC, Royer RJ, Steru L, Parot P. Double-blind randomized multicentre trial of acamprosate in maintaining abstinence from alcohol. Alcohol Alcohol. 1995;30(2):239-47.

47. Kiefer F, Mann K. Acamprosate: how, where, and for whom does it work? Mechanism of action, treatment targets, and individualized therapy. Curr Pharm Des. 2010;16(19):2098-102.

48. Covarrubias MY, Khan RL, Vadigepalli R, Hoek JB, Schwaber JS. Chronic alcohol exposure alters transcription broadly in a key integrative brain nucleus for homeostasis: the nucleus tractus solitarius. Physiol Genomics. 2005;24(1):45-58.

49. Ronis MJ, Wands JR, Badger TM, de la Monte SM, Lang CH, Calissendorff J. Alcohol-induced disruption of endocrine signaling. Alcohol Clin Exp Res. 2007;31(8):126985 .

50. Koob GF. Drugs of abuse: anatomy, pharmacology and function of reward pathways. Trends Pharmacol Sci. 1992;13(5):177-84.

51. Cowen MS, Lawrence AJ. The role of opioid-dopamine interactions in the induction and maintenance of ethanol consumption. Prog Neuropsychopharmacol Biol Psychiatry. 1999;23(7):1171-212.

52. Xiao C, Ye JH. Ethanol dually modulates GABAergic synaptic transmission onto dopaminergic neurons in ventral tegmental area: role of mu-opioid receptors. Neuroscience. 2008;153(1):240-8.

53. Holmes A, Spanagel R, Krystal JH. Glutamatergic targets for new alcohol medications. Psychopharmacology (Berl). 2013;229(3):539-54.

54. Mason BJ. Emerging pharmacotherapies for alcohol use disorder. Neuropharmacology. 2017;122:244-53.

55. Davies M. The role of GABAA receptors in mediating the effects of alcohol in the central nervous system. J Psychiatry Neurosci. 2003;28(4):263-74.

56. Grobin AC, Matthews DB, Devaud LL, Morrow AL. The role of GABAA receptors in the acute and chronic effects of ethanol. Psychopharmacology. 1998;139(1):2-19. 57. Kokka N, Sapp DW, Taylor AM, Olsen RW. The kindling model of alcohol dependence: similar persistent reduction in seizure threshold to pentylenetetrazol in animals receiving chronic ethanol or chronic pentylenetetrazol. Alcohol Clin Exp Res. 1993;17(3):52531.

58. Kang M, Spigelman I, Sapp DW, Olsen RW. Persistent reduction of GABA(A) receptor-mediated inhibition in rat hippocampus after chronic intermittent ethanol treatment. Brain Res. 1996;709(2):221-8.

59. Kalivas PW, Duffy P, Eberhardt H. Modulation of A10 dopamine neurons by gamma-aminobutyric acid agonists. J Pharmacol Exp Ther. 1990;253(2):858-66.

60. Gianoulakis C. Implications of endogenous opioids and dopamine in alcoholism: human and basic science studies. Alcohol Alcohol. 1996;31 Suppl 1:33-42.

61. Ferraguti F, Shigemoto R. Metabotropic glutamate receptors. Cell Tissue Res. 2006;326(2):483-504.

62. Ozawa S, Kamiya H, Tsuzuki K. Glutamate receptors in the mammalian central nervous system. Prog Neurobiol. 1998;54(5):581-618.

63. Danbolt NC. Glutamate uptake. Prog Neurobiol. 2001;65(1):1-105. 
64. Dahchour A, De Witte P. Ethanol and amino acids in the central nervous system: assessment of the pharmacological actions of acamprosate. Prog Neurobiol. 2000;60(4):34362.

65. Spanagel R, Pendyala G, Abarca C, Zghoul T, Sanchis-Segura C, Magnone MC, et al. The clock gene Per2 influences the glutamatergic system and modulates alcohol consumption. Nat Med. 2005;11(1):35-42.

$66 . \quad$ Pulvirenti L, Diana M. Drug dependence as a disorder of neural plasticity: focus on dopamine and glutamate. Rev Neurosci. 2001;12(2):141-58.

67. Lovinger DM, White G, Weight FF. Ethanol inhibits NMDA-activated ion current in hippocampal neurons. Science. 1989;243(4899):1721-4.

68. Bell RL, Hauser SR, McClintick J, Rahman S, Edenberg HJ, Szumlinski KK, et al. Ethanol-Associated Changes in Glutamate Reward Neurocircuitry: A Minireview of Clinical and Preclinical Genetic Findings. Prog Mol Biol Transl Sci. 2016;137:41-85.

69. Spanagel R, Vengeliene V, Jandeleit B, Fischer W-N, Grindstaff K, Zhang X, et al. Acamprosate produces its anti-relapse effects via calcium. Neuropsychopharmacology. 2014;39(4):783.

70. Schuster R, Koopmann A, Grosshans M, Reinhard I, Spanagel R, Kiefer F. Association of plasma calcium concentrations with alcohol craving: New data on potential pathways. Eur Neuropsychopharmacol. 2017;27(1):42-7.

71. Moghaddam B. Stress preferentially increases extraneuronal levels of excitatory amino acids in the prefrontal cortex: comparison to hippocampus and basal ganglia. $\mathrm{J}$ Neurochem. 1993;60(5):1650-7.

72. Hashimoto K, Sawa A, Iyo M. Increased levels of glutamate in brains from patients with mood disorders. Biol Psychiatry. 2007;62(11):1310-6.

73. Rajkowska G. Postmortem studies in mood disorders indicate altered numbers of neurons and glial cells. Biol Psychiatry. 2000;48(8):766-77.

74. Choudary PV, Molnar M, Evans SJ, Tomita H, Li JZ, Vawter MP, et al. Altered cortical glutamatergic and GABAergic signal transmission with glial involvement in depression. Proc Natl Acad Sci U S A. 2005;102(43):15653-8.

75. Russo SJ, Dietz DM, Dumitriu D, Morrison JH, Malenka RC, Nestler EJ. The addicted synapse: mechanisms of synaptic and structural plasticity in nucleus accumbens. Trends Neurosci. 2010;33(6):267-76.

76. Stuber GD, Sparta DR, Stamatakis AM, van Leeuwen WA, Hardjoprajitno JE, Cho $\mathrm{S}$, et al. Excitatory transmission from the amygdala to nucleus accumbens facilitates reward seeking. Nature. 2011;475(7356):377-80.

77. Chang HT, Kitai ST. Projection neurons of the nucleus accumbens: an intracellular labeling study. Brain Res. 1985;347(1):112-6.

78. Brownstein MJ, Mroz EA, Tappaz ML, Leeman SE. On the origin of substance $\mathrm{P}$ and glutamic acid decarboxylase (GAD) in the substantia nigra. Brain Res. 1977;135(2):31523.

79. Gerfen CR, Young WS, 3rd. Distribution of striatonigral and striatopallidal peptidergic neurons in both patch and matrix compartments: an in situ hybridization histochemistry and fluorescent retrograde tracing study. Brain Res. 1988;460(1):161-7.

80. Gerfen CR, Engber TM, Mahan LC, Susel Z, Chase TN, Monsma FJ, Jr., et al. D1 and D2 dopamine receptor-regulated gene expression of striatonigral and striatopallidal neurons. Science. 1990;250(4986):1429-32.

81. Gianoulakis C. Endogenous opioids and addiction to alcohol and other drugs of abuse. Current topics in medicinal chemistry. 2004;4(1):39-50. 
Oslin DW, Berrettini W, Kranzler HR, Pettinati H, Gelernter J, Volpicelli JR, et al. A functional polymorphism of the mu-opioid receptor gene is associated with naltrexone response in alcohol-dependent patients. Neuropsychopharmacology. 2003;28(8):1546-52.

83. Walker BM. Conceptualizing withdrawal-induced escalation of alcohol selfadministration as a learned, plasticity-dependent process. Alcohol. 2012;46(4):339-48.

84. Kissler JL, Walker BM. Dissociating Motivational From Physiological Withdrawal in Alcohol Dependence: Role of Central Amygdala kappa-Opioid Receptors. Neuropsychopharmacology. 2016;41(2):560-7.

85. Wee S, Koob GF. The role of the dynorphin-kappa opioid system in the reinforcing effects of drugs of abuse. Psychopharmacology (Berl). 2010;210(2):121-35.

86. Holter SM, Henniger MS, Lipkowski AW, Spanagel R. Kappa-opioid receptors and relapse-like drinking in long-term ethanol-experienced rats. Psychopharmacology (Berl). 2000;153(1):93-102.

87. Walker BM, Valdez GR, McLaughlin JP, Bakalkin G. Targeting dynorphin/kappa opioid receptor systems to treat alcohol abuse and dependence. Alcohol. 2012;46(4):359-70.

88. Kissler JL, Sirohi S, Reis DJ, Jansen HT, Quock RM, Smith DG, et al. The onetwo punch of alcoholism: role of central amygdala dynorphins/kappa-opioid receptors. Biol Psychiatry. 2014;75(10):774-82.

89. de Waele JP, Gianoulakis C. Characterization of the mu and delta opioid receptors in the brain of the C57BL/6 and DBA/2 mice, selected for their differences in voluntary ethanol consumption. Alcohol Clin Exp Res. 1997;21(4):754-62.

90. de Waele JP, Kiianmaa K, Gianoulakis C. Distribution of the mu and delta opioid binding sites in the brain of the alcohol-preferring AA and alcohol-avoiding ANA lines of rats. J Pharmacol Exp Ther. 1995;275(1):518-27.

91. Roberts AJ, Gold LH, Polis I, McDonald JS, Filliol D, Kieffer BL, et al. Increased ethanol self-administration in $\delta$-opioid receptor knockout mice. Alcoholism: Clinical and Experimental Research. 2001;25(9):1249-56.

92. Turchan J, Przewłocka B, Toth G, Lasoń W, Borsodi A, Przewłocki R. The effect of repeated administration of morphine, cocaine and ethanol on mu and delta opioid receptor density in the nucleus accumbens and striatum of the rat. Neuroscience. 1999;91(3):971-7.

93. McBride WJ, Li T-K. Animal models of alcoholism: neurobiology of high alcohol-drinking behavior in rodents. Critical Reviews ${ }^{\text {TM }}$ in Neurobiology. 1998;12(4).

94. Bowirrat A, Oscar-Berman M. Relationship between dopaminergic neurotransmission, alcoholism, and Reward Deficiency syndrome. Am J Med Genet B Neuropsychiatr Genet. 2005;132B(1):29-37.

95. Pacak K, Palkovits M. Stressor specificity of central neuroendocrine responses: implications for stress-related disorders. Endocr Rev. 2001;22(4):502-48.

96. Herman JP, Prewitt CM, Cullinan WE. Neuronal circuit regulation of the hypothalamo-pituitary-adrenocortical stress axis. Crit Rev Neurobiol. 1996;10(3-4):371-94.

97. Merchenthaler I, Vigh S, Petrusz P, Schally AV. Immunocytochemical localization of corticotropin-releasing factor $(\mathrm{CRF})$ in the rat brain. Am $J$ Anat. 1982;165(4):385-96.

98. Davis M. Are different parts of the extended amygdala involved in fear versus anxiety? Biol Psychiatry. 1998;44(12):1239-47.

99. Davis M, Walker DL, Miles L, Grillon C. Phasic vs sustained fear in rats and humans: role of the extended amygdala in fear vs anxiety. Neuropsychopharmacology. 2010;35(1):105-35.

100. Rodriguez FD, Covenas R. Targeting NPY, CRF/UCNs and NPS neuropeptide systems to treat Alcohol Use Disorder (AUD). Curr Med Chem. 2017. 
101. DiLeone RJ, Georgescu D, Nestler EJ. Lateral hypothalamic neuropeptides in reward and drug addiction. Life Sci. 2003;73(6):759-68.

102. Gilpin NW. Corticotropin-releasing factor (CRF) and neuropeptide Y (NPY): effects on inhibitory transmission in central amygdala, and anxiety- \& alcohol-related behaviors. Alcohol. 2012;46(4):329-37.

103. Lagerström MC, Schiöth HB. Structural diversity of G protein-coupled receptors and significance for drug discovery. Nature reviews Drug discovery. 2008;7(4):339.

104. Lefkowitz RJ. Historical review: a brief history and personal retrospective of seven-transmembrane receptors. Trends Pharmacol Sci. 2004;25(8):413-22.

105. Rosenbaum DM, Rasmussen SG, Kobilka BK. The structure and function of Gprotein-coupled receptors. Nature. 2009;459(7245):356-63.

106. Pitcher JA, Freedman NJ, Lefkowitz RJ. G protein-coupled receptor kinases. Annu Rev Biochem. 1998;67:653-92.

107. DeWire SM, Ahn S, Lefkowitz RJ, Shenoy SK. Beta-arrestins and cell signaling. Annu Rev Physiol. 2007;69:483-510.

108. Shukla AK, Xiao K, Lefkowitz RJ. Emerging paradigms of beta-arrestindependent seven transmembrane receptor signaling. Trends Biochem Sci. 2011;36(9):457-69.

109. Luttrell LM, Gesty-Palmer D. Beyond desensitization: physiological relevance of arrestin-dependent signaling. Pharmacol Rev. 2010;62(2):305-30.

110. Gainetdinov RR, Premont RT, Bohn LM, Lefkowitz RJ, Caron MG. Desensitization of $\mathrm{G}$ protein-coupled receptors and neuronal functions. Annu Rev Neurosci. 2004;27:107-44.

111. Bjork K, Tronci V, Thorsell A, Tanda G, Hirth N, Heilig M, et al. beta-Arrestin 2 knockout mice exhibit sensitized dopamine release and increased reward in response to a low dose of alcohol. Psychopharmacology (Berl). 2013;230(3):439-49.

112. Bjork K, Rimondini R, Hansson AC, Terasmaa A, Hyytia P, Heilig M, et al. Modulation of voluntary ethanol consumption by beta-arrestin 2. The FASEB journal : official publication of the Federation of American Societies for Experimental Biology. 2008;22(7):2552-60.

113. Li H, Tao Y, Ma L, Liu X, Ma L. beta-Arrestin-2 inhibits preference for alcohol in mice and suppresses Akt signaling in the dorsal striatum. Neurosci Bull. 2013;29(5):531-40. 114. Bohn LM, Lefkowitz RJ, Gainetdinov RR, Peppel K, Caron MG, Lin FT. Enhanced morphine analgesia in mice lacking beta-arrestin 2. Science. 1999;286(5449):24958 .

115. Bohn LM, Gainetdinov RR, Sotnikova TD, Medvedev IO, Lefkowitz RJ, Dykstra LA, et al. Enhanced rewarding properties of morphine, but not cocaine, in beta(arrestin)-2 knock-out mice. J Neurosci. 2003;23(32):10265-73.

116. Violin JD, Crombie AL, Soergel DG, Lark MW. Biased ligands at G-proteincoupled receptors: promise and progress. Trends Pharmacol Sci. 2014;35(7):308-16.

117. Mollereau C, Parmentier M, Mailleux P, Butour JL, Moisand C, Chalon P, et al. ORL1, a novel member of the opioid receptor family. Cloning, functional expression and localization. FEBS letters. 1994;341(1):33-8.

118. Meunier JC, Mollereau C, Toll L, Suaudeau C, Moisand C, Alvinerie P, et al. Isolation and structure of the endogenous agonist of opioid receptor-like ORL1 receptor. Nature. 1995;377(6549):532-5.

119. Reinscheid RK, Nothacker HP, Bourson A, Ardati A, Henningsen RA, Bunzow JR, et al. Orphanin FQ: a neuropeptide that activates an opioidlike $G$ protein-coupled receptor. Science. 1995;270(5237):792-4.

120. Julius D. Pharmacology. Home for an orphan endorphin. Nature. 1995;377(6549):476. 
121. Reinscheid RK, Ardati A, Monsma FJ, Jr., Civelli O. Structure-activity relationship studies on the novel neuropeptide orphanin FQ. J Biol Chem. 1996;271(24):14163-8.

122. Darland T, Heinricher MM, Grandy DK. Orphanin FQ/nociceptin: a role in pain and analgesia, but so much more. Trends Neurosci. 1998;21(5):215-21.

123. Sim LJ, Childers SR. Anatomical distribution of mu, delta, and kappa opioid- and nociceptin/orphanin FQ-stimulated [35S]guanylyl-5'-O-(gamma-thio)-triphosphate binding in guinea pig brain. J Comp Neurol. 1997;386(4):562-72.

124. Anton B, Fein J, To T, Li X, Silberstein L, Evans CJ. Immunohistochemical localization of ORL-1 in the central nervous system of the rat. The Journal of comparative neurology. 1996;368(2):229-51.

125. Neal CR, Jr., Mansour A, Reinscheid R, Nothacker HP, Civelli O, Watson SJ, Jr. Localization of orphanin FQ (nociceptin) peptide and messenger RNA in the central nervous system of the rat. The Journal of comparative neurology. 1999;406(4):503-47.

126. Berthele A, Platzer S, Dworzak D, Schadrack J, Mahal B, Buttner A, et al. [3H]nociceptin ligand-binding and nociceptin opioid receptor mrna expression in the human brain. Neuroscience. 2003;121(3):629-40.

127. Liu Z, Wang Y, Zhang J, Ding J, Guo L, Cui D, et al. Orphanin FQ: an endogenous antagonist of rat brain dopamine transporter. Neuroreport. 2001;12(4):699-702.

128. Lambert DG. The nociceptin/orphanin FQ receptor: a target with broad therapeutic potential. Nat Rev Drug Discov. 2008;7(8):694-710.

129. Zaveri NT. The nociceptin/orphanin FQ receptor (NOP) as a target for drug abuse medications. Curr Top Med Chem. 2011;11(9):1151-6.

130. Mogil JS, Grisel JE, Reinscheid RK, Civelli O, Belknap JK, Grandy DK. Orphanin FQ is a functional anti-opioid peptide. Neuroscience. 1996;75(2):333-7.

131. Morgan MM, Grisel JE, Robbins CS, Grandy DK. Antinociception mediated by the periaqueductal gray is attenuated by orphanin FQ. Neuroreport. 1997;8(16):3431-4.

132. Noda Y, Mamiya T, Nabeshima T, Nishi M, Higashioka M, Takeshima H. Loss of antinociception induced by naloxone benzoylhydrazone in nociceptin receptor-knockout mice. J Biol Chem. 1998;273(29):18047-51.

133. Gunther T, Dasgupta P, Mann A, Miess E, Kliewer A, Fritzwanker S, et al. Targeting multiple opioid receptors - improved analgesics with reduced side effects? $\mathrm{Br} \mathbf{J}$ Pharmacol. 2017.

134. Meunier JC. Nociceptin/orphanin FQ and the opioid receptor-like ORL1 receptor. Eur J Pharmacol. 1997;340(1):1-15.

135. Lapalu S, Moisand C, Mazarguil H, Cambois G, Mollereau C, Meunier JC. Comparison of the structure-activity relationships of nociceptin and dynorphin A using chimeric peptides. FEBS Lett. 1997;417(3):333-6.

136. Reinscheid RK, Higelin J, Henningsen RA, Monsma FJ, Jr., Civelli O. Structures that delineate orphanin FQ and dynorphin A pharmacological selectivities. J Biol Chem. 1998;273(3):1490-5.

137. Wagner EJ, Ronnekleiv OK, Grandy DK, Kelly MJ. The peptide orphanin FQ inhibits beta-endorphin neurons and neurosecretory cells in the hypothalamic arcuate nucleus by activating an inwardly-rectifying K+ conductance. Neuroendocrinology. 1998;67(2):73-82. 138. Herz A. Endogenous opioid systems and alcohol addiction. Psychopharmacology (Berl). 1997;129(2):99-111.

139. Ciccocioppo R, Panocka I, Polidori C, Regoli D, Massi M. Effect of nociceptin on alcohol intake in alcohol-preferring rats. Psychopharmacology. 1999;141(2):220-4.

140. Ciccocioppo R, Economidou D, Fedeli A, Angeletti S, Weiss F, Heilig M, et al. Attenuation of ethanol self-administration and of conditioned reinstatement of alcohol-seeking 
behaviour by the antiopioid peptide nociceptin/orphanin FQ in alcohol-preferring rats. Psychopharmacology. 2004;172(2):170-8.

141. Economidou D, Fedeli A, Fardon RM, Weiss F, Massi M, Ciccocioppo R. Effect of novel nociceptin/orphanin FQ-NOP receptor ligands on ethanol drinking in alcoholpreferring msP rats. Peptides. 2006;27(12):3299-306.

142. Jenck F, Wichmann J, Dautzenberg FM, Moreau JL, Ouagazzal AM, Martin JR, et al. A synthetic agonist at the orphanin FQ/nociceptin receptor ORL1: anxiolytic profile in the rat. Proc Natl Acad Sci U S A. 2000;97(9):4938-43.

143. Kuzmin A, Kreek MJ, Bakalkin G, Liljequist S. The nociceptin/orphanin FQ receptor agonist Ro 64-6198 reduces alcohol self-administration and prevents relapse-like alcohol drinking. Neuropsychopharmacology : official publication of the American College of Neuropsychopharmacology. 2007;32(4):902-10.

144. Marquez P, Nguyen AT, Hamid A, Lutfy K. The endogenous OFQ/N/ORL-1 receptor system regulates the rewarding effects of acute cocaine. Neuropharmacology. 2008;54(3):564-8.

145. Sakoori K, Murphy NP. Endogenous nociceptin (orphanin FQ) suppresses basal hedonic state and acute reward responses to methamphetamine and ethanol, but facilitates chronic responses. Neuropsychopharmacology. 2008;33(4):877-91.

146. Murphy NP, Ly HT, Maidment NT. Intracerebroventricular orphanin $\mathrm{FQ}$ /nociceptin suppresses dopamine release in the nucleus accumbens of anaesthetized rats. Neuroscience. 1996;75(1):1-4.

147. Devine DP, Reinscheid RK, Monsma FJ, Jr., Civelli O, Akil H. The novel neuropeptide orphanin FQ fails to produce conditioned place preference or aversion. Brain research. 1996;727(1-2):225-9.

148. Köster A, Montkowski A, Schulz S, Stübe E-M, Knaudt K, Jenck F, et al. Targeted disruption of the orphanin FQ/nociceptin gene increases stress susceptibility and impairs stress adaptation in mice. Proceedings of the National Academy of Sciences. 1999;96(18):10444-9.

149. Martin-Fardon R, Zorrilla EP, Ciccocioppo R, Weiss F. Role of innate and druginduced dysregulation of brain stress and arousal systems in addiction: Focus on corticotropinreleasing factor, nociceptin/orphanin FQ, and orexin/hypocretin. Brain research. 2010;1314:145-61.

150. Rutten K, De Vry J, Bruckmann W, Tzschentke TM. Effects of the NOP receptor agonist Ro65-6570 on the acquisition of opiate- and psychostimulant-induced conditioned place preference in rats. European journal of pharmacology. 2010;645(1-3):119-26.

151. Ciccocioppo R, Angeletti S, Panocka I, Massi M. Nociceptin/orphanin FQ and drugs of abuse. Peptides. 2000;21(7):1071-80.

152. Maidment NT, Chen Y, Tan AM, Murphy NP, Leslie FM. Rat ventral midbrain dopamine neurons express the orphanin FQ/nociceptin receptor ORL-1. Neuroreport. 2002;13(9):1137-40.

153. Roberto M, Madamba SG, Moore SD, Tallent MK, Siggins GR. Ethanol increases GABAergic transmission at both pre- and postsynaptic sites in rat central amygdala neurons. Proc Natl Acad Sci U S A. 2003;100(4):2053-8.

154. Roberto M, Madamba SG, Stouffer DG, Parsons LH, Siggins GR. Increased GABA release in the central amygdala of ethanol-dependent rats. $J$ Neurosci. 2004;24(45):10159-66.

155. Kuzmin A, Sandin J, Terenius L, Ogren SO. Acquisition, expression, and reinstatement of ethanol-induced conditioned place preference in mice: effects of opioid receptor-like 1 receptor agonists and naloxone. J Pharmacol Exp Ther. 2003;304(1):310-8. 
156. Boothby LA, Doering PL. Buprenorphine for the treatment of opioid dependence. Am J Health Syst Pharm. 2007;64(3):266-72.

157. Pergolizzi J, Aloisi AM, Dahan A, Filitz J, Langford R, Likar R, et al. Current knowledge of buprenorphine and its unique pharmacological profile. Pain Pract. 2010;10(5):428-50.

158. Lutfy K, Eitan S, Bryant CD, Yang YC, Saliminejad N, Walwyn W, et al. Buprenorphine-induced antinociception is mediated by mu-opioid receptors and compromised by concomitant activation of opioid receptor-like receptors. J Neurosci. 2003;23(32):10331-7. 159. Barrot M, Marinelli M, Abrous DN, Rouge-Pont F, Le Moal M, Piazza PV. Functional heterogeneity in dopamine release and in the expression of Fos-like proteins within the rat striatal complex. Eur J Neurosci. 1999;11(4):1155-66.

160. Ciccocioppo R, Economidou D, Rimondini R, Sommer W, Massi M, Heilig M. Buprenorphine reduces alcohol drinking through activation of the nociceptin/orphanin FQNOP receptor system. Biol Psychiatry. 2007;61(1):4-12.

161. Tatemoto K, Carlquist M, Mutt V. Neuropeptide Y--a novel brain peptide with structural similarities to peptide YY and pancreatic polypeptide. Nature. 1982;296(5858):65960 .

162. Adrian TE, Allen JM, Bloom SR, Ghatei MA, Rossor MN, Roberts GW, et al. Neuropeptide Y distribution in human brain. Nature. 1983;306(5943):584-6.

163. Heilig M, Widerlov E. Neurobiology and clinical aspects of neuropeptide Y. Crit Rev Neurobiol. 1995;9(2-3):115-36.

164. Heilig M, Widerlov E. Neuropeptide Y: an overview of central distribution, functional aspects, and possible involvement in neuropsychiatric illnesses. Acta Psychiatr Scand. 1990;82(2):95-114.

165. Gerald C, Walker MW, Criscione L, Gustafson EL, Batzl-Hartmann C, Smith $\mathrm{KE}$, et al. A receptor subtype involved in neuropeptide-Y-induced food intake. Nature. 1996;382(6587):168-71.

166. Broqua P, Wettstein JG, Rocher MN, Gauthier-Martin B, Junien JL. Behavioral effects of neuropeptide $\mathrm{Y}$ receptor agonists in the elevated plus-maze and fear-potentiated startle procedures. Behav Pharmacol. 1995;6(3):215-22.

167. Thorsell A, Michalkiewicz M, Dumont Y, Quirion R, Caberlotto L, Rimondini $\mathrm{R}$, et al. Behavioral insensitivity to restraint stress, absent fear suppression of behavior and impaired spatial learning in transgenic rats with hippocampal neuropeptide Y overexpression. Proc Natl Acad Sci U S A. 2000;97(23):12852-7.

168. Heilig M, McLeod S, Brot M, Heinrichs SC, Menzaghi F, Koob GF, et al. Anxiolytic-like action of neuropeptide $\mathrm{Y}$ : mediation by $\mathrm{Y} 1$ receptors in amygdala, and dissociation from food intake effects. Neuropsychopharmacology. 1993;8(4):357-63.

169. Heilig M, Soderpalm B, Engel JA, Widerlov E. Centrally administered neuropeptide Y (NPY) produces anxiolytic-like effects in animal anxiety models. Psychopharmacology. 1989;98(4):524-9.

170. Woldbye DP, Nanobashvili A, Husum H, Bolwig TG, Kokaia M. Neuropeptide $\mathrm{Y}$ inhibits in vitro epileptiform activity in the entorhinal cortex of mice. Neurosci Lett. 2002;333(2):127-30.

171. Woldbye DP, Nanobashvili A, Sorensen AT, Husum H, Bolwig TG, Sorensen G, et al. Differential suppression of seizures via Y2 and Y5 neuropeptide Y receptors. Neurobiol Dis. 2005;20(3):760-72.

172. Vezzani A, Sperk G, Colmers WF. Neuropeptide Y: emerging evidence for a functional role in seizure modulation. Trends Neurosci. 1999;22(1):25-30.

173. Heilig M. The NPY system in stress, anxiety and depression. Neuropeptides. 2004;38(4):213-24. 
174.

Heilig M, Zachrisson O, Thorsell A, Ehnvall A, Mottagui-Tabar S, Sjogren M, et al. Decreased cerebrospinal fluid neuropeptide Y (NPY) in patients with treatment refractory unipolar major depression: preliminary evidence for association with preproNPY gene polymorphism. J Psychiatr Res. 2004;38(2):113-21.

175. Thorsell A, Carlsson K, Ekman R, Heilig M. Behavioral and endocrine adaptation, and up-regulation of NPY expression in rat amygdala following repeated restraint stress. Neuroreport. 1999;10(14):3003-7.

176. Gilpin NW. Neuropeptide Y (NPY) in the extended amygdala is recruited during the transition to alcohol dependence. Neuropeptides. 2012;46(6):253-9.

177. Parker RM, Herzog H. Regional distribution of Y-receptor subtype mRNAs in rat brain. Eur J Neurosci. 1999;11(4):1431-48.

178. Michel MC, Beck-Sickinger A, Cox H, Doods HN, Herzog H, Larhammar D, et al. XVI. International Union of Pharmacology recommendations for the nomenclature of neuropeptide Y, peptide YY, and pancreatic polypeptide receptors. Pharmacol Rev. 1998;50(1):143-50.

179. King PJ, Widdowson PS, Doods HN, Williams G. Regulation of neuropeptide Y release by neuropeptide $\mathrm{Y}$ receptor ligands and calcium channel antagonists in hypothalamic slices. J Neurochem. 1999;73(2):641-6.

180. Colmers WF, Lukowiak K, Pittman QJ. Neuropeptide Y action in the rat hippocampal slice: site and mechanism of presynaptic inhibition. J Neurosci. 1988;8(10):382737.

181. Chen G, van den Pol AN. Multiple NPY receptors coexist in pre- and postsynaptic sites: inhibition of GABA release in isolated self-innervating SCN neurons. J Neurosci. 1996;16(23):7711-24.

182. Colmers WF, Klapstein GJ, Fournier A, St-Pierre S, Treherne KA. Presynaptic inhibition by neuropeptide $\mathrm{Y}$ in rat hippocampal slice in vitro is mediated by a $\mathrm{Y} 2$ receptor. $\mathrm{Br}$ J Pharmacol. 1991;102(1):41-4.

183. Redrobe JP, Dumont Y, Herzog H, Quirion R. Neuropeptide Y (NPY) Y2 receptors mediate behaviour in two animal models of anxiety: evidence from Y2 receptor knockout mice. Behav Brain Res. 2003;141(2):251-5.

184. Tschenett A, Singewald N, Carli M, Balducci C, Salchner P, Vezzani A, et al. Reduced anxiety and improved stress coping ability in mice lacking NPY-Y2 receptors. Eur J Neurosci. 2003;18(1):143-8.

185. Nakajima M, Inui A, Asakawa A, Momose K, Ueno N, Teranishi A, et al. Neuropeptide Y produces anxiety via Y2-type receptors. Peptides. 1998;19(2):359-63.

186. Sajdyk TJ, Schober DA, Gehlert DR. Neuropeptide Y receptor subtypes in the basolateral nucleus of the amygdala modulate anxiogenic responses in rats. Neuropharmacology. 2002;43(7):1165-72.

187. Brothers SP, Saldanha SA, Spicer TP, Cameron M, Mercer BA, Chase P, et al. Selective and brain penetrant neuropeptide y y2 receptor antagonists discovered by whole-cell high-throughput screening. Mol Pharmacol. 2010;77(1):46-57.

188. Cippitelli A, Rezvani AH, Robinson JE, Eisenberg L, Levin ED, Bonaventure P, et al. The novel, selective, brain-penetrant neuropeptide Y Y2 receptor antagonist, JNJ31020028 , tested in animal models of alcohol consumption, relapse, and anxiety. Alcohol. 2011;45(6):567-76.

189. Thorsell A, Rimondini R, Heilig M. Blockade of central neuropeptide Y (NPY) Y2 receptors reduces ethanol self-administration in rats. Neurosci Lett. 2002;332(1):1-4.

190. Rimondini R, Thorsell A, Heilig M. Suppression of ethanol self-administration by the neuropeptide Y (NPY) Y2 receptor antagonist BIIE0246: evidence for sensitization in rats with a history of dependence. Neurosci Lett. 2005;375(2):129-33. 
191. Heilig M, Thorsell A. Brain neuropeptide Y (NPY) in stress and alcohol dependence. Rev Neurosci. 2002;13(1):85-94.

192. Thorsell A. Neuropeptide Y (NPY) in alcohol intake and dependence. Peptides. 2007;28(2):480-3.

193. Thiele TE, Marsh DJ, Ste Marie L, Bernstein IL, Palmiter RD. Ethanol consumption and resistance are inversely related to neuropeptide $\mathrm{Y}$ levels. Nature. 1998;396(6709):366-9.

194. Hwang BH, Suzuki R, Lumeng L, Li TK, McBride WJ. Innate differences in neuropeptide Y (NPY) mRNA expression in discrete brain regions between alcohol-preferring (P) and -nonpreferring (NP) rats: a significantly low level of NPY mRNA in dentate gyrus of the hippocampus and absence of NPY mRNA in the medial habenular nucleus of $\mathrm{P}$ rats. Neuropeptides. 2004;38(6):359-68.

195. Thiele TE, Badia-Elder NE. A role for neuropeptide Y in alcohol intake control: evidence from human and animal research. Physiol Behav. 2003;79(1):95-101.

196. Olling JD, Ulrichsen J, Haugbol S, Glenthoj B, Hemmingsen R, Woldbye DP. Decreased gene expression of neuropeptide $\mathrm{Y}$ and its receptors in hippocampal regions during ethanol withdrawal in rats. Neurosci Lett. 2007;424(3):160-4.

197. Ilveskoski E, Kajander OA, Lehtimaki T, Kunnas T, Karhunen PJ, Heinala P, et al. Association of neuropeptide y polymorphism with the occurrence of type 1 and type 2 alcoholism. Alcohol Clin Exp Res. 2001;25(10):1420-2.

198. Mottagui-Tabar S, Prince JA, Wahlestedt C, Zhu G, Goldman D, Heilig M. A novel single nucleotide polymorphism of the neuropeptide Y (NPY) gene associated with alcohol dependence. Alcohol Clin Exp Res. 2005;29(5):702-7.

199. Heilig M, McLeod S, Koob GK, Britton KT. Anxiolytic-like effect of neuropeptide Y (NPY), but not other peptides in an operant conflict test. Regul Pept. 1992;41(1):61-9.

200. Bannon AW, Seda J, Carmouche M, Francis JM, Norman MH, Karbon B, et al. Behavioral characterization of neuropeptide Y knockout mice. Brain Res. 2000;868(1):79-87. 201. Thorsell A, Svensson P, Wiklund L, Sommer W, Ekman R, Heilig M. Suppressed neuropeptide Y (NPY) mRNA in rat amygdala following restraint stress. Regul Pept. 1998;75$76: 247-54$.

202. Silva AP, Xapelli S, Pinheiro PS, Ferreira R, Lourenco J, Cristovao A, et al. Upregulation of neuropeptide $\mathrm{Y}$ levels and modulation of glutamate release through neuropeptide $\mathrm{Y}$ receptors in the hippocampus of kainate-induced epileptic rats. $\mathrm{J}$ Neurochem. 2005;93(1):163-70.

203. Sajdyk TJ, Shekhar A, Gehlert DR. Interactions between NPY and CRF in the amygdala to regulate emotionality. Neuropeptides. 2004;38(4):225-34.

204. Sun QQ, Akk G, Huguenard JR, Prince DA. Differential regulation of GABA release and neuronal excitability mediated by neuropeptide $\mathrm{Y} 1$ and $\mathrm{Y} 2$ receptors in rat thalamic neurons. J Physiol. 2001;531(Pt 1):81-94.

205. Karl T, Burne TH, Herzog H. Effect of Y1 receptor deficiency on motor activity, exploration, and anxiety. Behav Brain Res. 2006;167(1):87-93.

206. Mathe AA, Jimenez PA, Theodorsson E, Stenfors C. Neuropeptide Y, neurokinin A and neurotensin in brain regions of Fawn Hooded "depressed", Wistar, and Sprague Dawley rats. Effects of electroconvulsive stimuli. Prog Neuropsychopharmacol Biol Psychiatry. 1998;22(3):529-46.

207. Jimenez-Vasquez PA, Diaz-Cabiale Z, Caberlotto L, Bellido I, Overstreet D, Fuxe K, et al. Electroconvulsive stimuli selectively affect behavior and neuropeptide Y (NPY) and NPY Y(1) receptor gene expressions in hippocampus and hypothalamus of Flinders Sensitive Line rat model of depression. Eur Neuropsychopharmacol. 2007;17(4):298-308. 
208. Ishida H, Shirayama Y, Iwata M, Katayama S, Yamamoto A, Kawahara R, et al. Infusion of neuropeptide $\mathrm{Y}$ into $\mathrm{CA} 3$ region of hippocampus produces antidepressant-like effect via Y1 receptor. Hippocampus. 2007;17(4):271-80.

209. Howell OW, Silva S, Scharfman HE, Sosunov AA, Zaben M, Shtaya A, et al. Neuropeptide $\mathrm{Y}$ is important for basal and seizure-induced precursor cell proliferation in the hippocampus. Neurobiol Dis. 2007;26(1):174-88.

210. Nikisch G, Agren H, Eap CB, Czernik A, Baumann P, Mathe AA. Neuropeptide $\mathrm{Y}$ and corticotropin-releasing hormone in CSF mark response to antidepressive treatment with citalopram. Int J Neuropsychopharmacol. 2005;8(3):403-10.

211. Nikisch G, Mathe AA. CSF monoamine metabolites and neuropeptides in depressed patients before and after electroconvulsive therapy. Eur Psychiatry. 2008;23(5):3569.

212. Heilig M, Egli M. Pharmacological treatment of alcohol dependence: target symptoms and target mechanisms. Pharmacol Ther. 2006;111(3):855-76.

213. Zhou Z, Zhu G, Hariri AR, Enoch MA, Scott D, Sinha R, et al. Genetic variation in human NPY expression affects stress response and emotion. Nature. 2008;452(7190):9971001 .

214. Kawauchi H, Kawazoe I, Tsubokawa M, Kishida M, Baker BI. Characterization of melanin-concentrating hormone in chum salmon pituitaries. Nature. 1983;305(5932):321-3.

215. Vaughan JM, Fischer WH, Hoeger C, Rivier J, Vale W. Characterization of melanin-concentrating hormone from rat hypothalamus. Endocrinology. 1989;125(3):1660-5.

216. Presse F, Nahon JL, Fischer WH, Vale W. Structure of the human melanin concentrating hormone mRNA. Mol Endocrinol. 1990;4(4):632-7.

217. Pissios P, Bradley RL, Maratos-Flier E. Expanding the scales: The multiple roles of $\mathrm{MCH}$ in regulating energy balance and other biological functions. Endocr Rev. 2006;27(6):606-20.

218. Bittencourt JC, Presse F, Arias C, Peto C, Vaughan J, Nahon JL, et al. The melanin-concentrating hormone system of the rat brain: an immuno- and hybridization histochemical characterization. J Comp Neurol. 1992;319(2):218-45.

219. Tan CP, Sano H, Iwaasa H, Pan J, Sailer AW, Hreniuk DL, et al. Melaninconcentrating hormone receptor subtypes 1 and 2: species-specific gene expression. Genomics. 2002;79(6):785-92.

220. Saito Y, Nothacker HP, Wang Z, Lin SH, Leslie F, Civelli O. Molecular characterization of the melanin-concentrating-hormone receptor. Nature. 1999;400(6741):2659.

221. Saito Y, Cheng M, Leslie FM, Civelli O. Expression of the melanin-concentrating hormone $(\mathrm{MCH})$ receptor mRNA in the rat brain. J Comp Neurol. 2001;435(1):26-40.

222. Hawes BE, Kil E, Green B, O'Neill K, Fried S, Graziano MP. The melaninconcentrating hormone receptor couples to multiple $\mathrm{G}$ proteins to activate diverse intracellular signaling pathways. Endocrinology. 2000;141(12):4524-32.

223. Chung S, Saito Y, Civelli O. MCH receptors/gene structure-in vivo expression. Peptides. 2009;30(11):1985-9.

224. Sailer AW, Sano H, Zeng Z, McDonald TP, Pan J, Pong SS, et al. Identification and characterization of a second melanin-concentrating hormone receptor, $\mathrm{MCH}-2 \mathrm{R}$. Proc Natl Acad Sci U S A. 2001;98(13):7564-9.

225. Obici S, Zhang BB, Karkanias G, Rossetti L. Hypothalamic insulin signaling is required for inhibition of glucose production. Nat Med. 2002;8(12):1376-82.

226. Elias CF, Saper CB, Maratos-Flier E, Tritos NA, Lee C, Kelly J, et al. Chemically defined projections linking the mediobasal hypothalamus and the lateral hypothalamic area. $\mathrm{J}$ Comp Neurol. 1998;402(4):442-59. 
227.

Della-Zuana O, Presse F, Ortola C, Duhault J, Nahon JL, Levens N. Acute and chronic administration of melanin-concentrating hormone enhances food intake and body weight in Wistar and Sprague-Dawley rats. Int J Obes Relat Metab Disord. 2002;26(10):128995 .

228. Ludwig DS, Tritos NA, Mastaitis JW, Kulkarni R, Kokkotou E, Elmquist J, et al. Melanin-concentrating hormone overexpression in transgenic mice leads to obesity and insulin resistance. J Clin Invest. 2001;107(3):379-86.

229. Tavares FX, Al-Barazanji KA, Bishop MJ, Britt CS, Carlton DL, Cooper JP, et al. 6-(4-chlorophenyl)-3-substituted-thieno[3,2-d]pyrimidin-4(3H)-one-based melaninconcentrating hormone receptor 1 antagonist. J Med Chem. 2006;49(24):7108-18.

230. Abbott CR, Kennedy AR, Wren AM, Rossi M, Murphy KG, Seal LJ, et al. Identification of hypothalamic nuclei involved in the orexigenic effect of melaninconcentrating hormone. Endocrinology. 2003;144(9):3943-9.

231. Georgescu D, Sears RM, Hommel JD, Barrot M, Bolanos CA, Marsh DJ, et al. The hypothalamic neuropeptide melanin-concentrating hormone acts in the nucleus accumbens to modulate feeding behavior and forced-swim performance. J Neurosci. 2005;25(11):293340 .

232. Wise RA, Rompre PP. Brain dopamine and reward. Annu Rev Psychol. 1989;40:191-225.

233. Colantuoni C, Rada P, McCarthy J, Patten C, Avena NM, Chadeayne A, et al. Evidence that intermittent, excessive sugar intake causes endogenous opioid dependence. Obes Res. 2002;10(6):478-88.

234. Zhang M, Balmadrid C, Kelley AE. Nucleus accumbens opioid, GABaergic, and dopaminergic modulation of palatable food motivation: contrasting effects revealed by a progressive ratio study in the rat. Behav Neurosci. 2003;117(2):202-11.

235. Avena NM, Rada P, Hoebel BG. Sugar bingeing in rats. Curr Protoc Neurosci. 2006; Chapter 9:Unit9 23C.

236. Avena NM, Rada P, Hoebel BG. Evidence for sugar addiction: behavioral and neurochemical effects of intermittent, excessive sugar intake. Neurosci Biobehav Rev. 2008;32(1):20-39.

237. Colantuoni C, Schwenker J, McCarthy J, Rada P, Ladenheim B, Cadet JL, et al. Excessive sugar intake alters binding to dopamine and mu-opioid receptors in the brain. Neuroreport. 2001;12(16):3549-52.

238. Volkow ND, Wang GJ, Fowler JS, Tomasi D, Baler R. Food and drug reward: overlapping circuits in human obesity and addiction. Curr Top Behav Neurosci. 2012;11:1-24. 239. Maldonado-Irizarry CS, Swanson CJ, Kelley AE. Glutamate receptors in the nucleus accumbens shell control feeding behavior via the lateral hypothalamus. J Neurosci. 1995;15(10):6779-88.

240. Hillebrand JJ, de Wied D, Adan RA. Neuropeptides, food intake and body weight regulation: a hypothalamic focus. Peptides. 2002;23(12):2283-306.

241. Schwartz MW, Woods SC, Porte D, Jr., Seeley RJ, Baskin DG. Central nervous system control of food intake. Nature. 2000;404(6778):661-71.

242. Aston-Jones G, Smith RJ, Moorman DE, Richardson KA. Role of lateral hypothalamic orexin neurons in reward processing and addiction. Neuropharmacology. 2009;56 Suppl 1:112-21.

243. Boutrel B, de Lecea L. Addiction and arousal: the hypocretin connection. Physiol Behav. 2008;93(4-5):947-51.

244. Kiefer F, Wiedemann K. Neuroendocrine pathways of addictive behaviour. Addict Biol. 2004;9(3-4):205-12. 
245.

Jezova D, Bartanusz V, Westergren I, Johansson BB, Rivier J, Vale W, et al. Rat melanin-concentrating hormone stimulates adrenocorticotropin secretion: evidence for a site of action in brain regions protected by the blood-brain barrier. Endocrinology. 1992;130(2):1024-9.

246. Kennedy AR, Todd JF, Dhillo WS, Seal LJ, Ghatei MA, O'Toole CP, et al. Effect of direct injection of melanin-concentrating hormone into the paraventricular nucleus: further evidence for a stimulatory role in the adrenal axis via SLC-1. J Neuroendocrinol. 2003;15(3):268-72.

247. Pariante CM, Lightman SL. The HPA axis in major depression: classical theories and new developments. Trends Neurosci. 2008;31(9):464-8.

248. Kela J, Salmi P, Rimondini-Giorgini R, Heilig M, Wahlestedt C. Behavioural analysis of melanin-concentrating hormone in rats: evidence for orexigenic and anxiolytic properties. Regul Pept. 2003;114(2-3):109-14.

249. Monzon ME, De Barioglio SR. Response to novelty after i.c.v. injection of melanin-concentrating hormone (MCH) in rats. Physiol Behav. 1999;67(5):813-7.

250. Borowsky B, Durkin MM, Ogozalek K, Marzabadi MR, DeLeon J, Lagu B, et al. Antidepressant, anxiolytic and anorectic effects of a melanin-concentrating hormone-1 receptor antagonist. Nat Med. 2002;8(8):825-30.

251. Gehlert DR, Rasmussen K, Shaw J, Li X, Ardayfio P, Craft L, et al. Preclinical evaluation of melanin-concentrating hormone receptor 1 antagonism for the treatment of obesity and depression. J Pharmacol Exp Ther. 2009;329(2):429-38.

252. Smith DG, Davis RJ, Rorick-Kehn L, Morin M, Witkin JM, McKinzie DL, et al. Melanin-concentrating hormone-1 receptor modulates neuroendocrine, behavioral, and corticolimbic neurochemical stress responses in mice. Neuropsychopharmacology. 2006;31(6):1135-45.

253. Kowalski TJ, McBriar MD. Therapeutic potential of melanin-concentrating hormone-1 receptor antagonists for the treatment of obesity. Expert Opin Investig Drugs. 2004;13(9):1113-22.

254. Mancini MC, Halpern A. Investigational therapies in the treatment of obesity. Expert Opin Investig Drugs. 2006;15(8):897-915.

255. Chung S, Hopf FW, Nagasaki H, Li CY, Belluzzi JD, Bonci A, et al. The melaninconcentrating hormone system modulates cocaine reward. Proc Natl Acad Sci U S A. 2009;106(16):6772-7.

256. Tyhon A, Lakaye B, Adamantidis A, Tirelli E. Amphetamine- and cocaineinduced conditioned place preference and concomitant psychomotor sensitization in mice with genetically inactivated melanin-concentrating hormone $\mathrm{MCH}(1)$ receptor. Eur J Pharmacol. 2008;599(1-3):72-80.

257. Duncan EA, Rider TR, Jandacek RJ, Clegg DJ, Benoit SC, Tso P, et al. The regulation of alcohol intake by melanin-concentrating hormone in rats. Pharmacol Biochem Behav. 2006;85(4):728-35.

258. Duncan EA, Sorrell JE, Adamantidis A, Rider T, Jandacek RJ, Seeley RJ, et al. Alcohol drinking in MCH receptor-1-deficient mice. Alcohol Clin Exp Res. 2007;31(8):132537.

259. Squeglia LM, Gray KM. Alcohol and Drug Use and the Developing Brain. Curr Psychiatry Rep. 2016;18(5):46.

260. Wiers CE, Cabrera E, Skarda E, Volkow ND, Wang GJ. PET imaging for addiction medicine: From neural mechanisms to clinical considerations. Prog Brain Res. 2016;224:175-201.

261. Flecknell P. Replacement, reduction and refinement. ALTEX. 2002;19(2):73-8. 
262. Tannenbaum J, Bennett BT. Russell and Burch's 3Rs then and now: the need for clarity in definition and purpose. J Am Assoc Lab Anim Sci. 2015;54(2):120-32.

263. Gazzaniga MS. The cognitive neurosciences: MIT press; 2004.

264. Pierce RC, O'Brien CP, Kenny PJ, Vanderschuren LJ. Rational development of addiction pharmacotherapies: successes, failures, and prospects. Cold Spring Harb Perspect Med. 2012;2(6):a012880.

$265 . \quad$ Robbins TW, Sahakian BJ. "Paradoxical" effects of psychomotor stimulant drugs in hyperactive children from the standpoint of behavioural pharmacology. Neuropharmacology. 1979;18(12):931-50.

266. Crawley JN. Exploratory behavior models of anxiety in mice. Neurosci Biobehav Rev. 1985;9(1):37-44.

267. Prut L, Belzung C. The open field as a paradigm to measure the effects of drugs on anxiety-like behaviors: a review. Eur J Pharmacol. 2003;463(1-3):3-33.

268. Lister RG. The use of a plus-maze to measure anxiety in the mouse. Psychopharmacology (Berl). 1987;92(2):180-5.

269. Shepherd JK, Grewal SS, Fletcher A, Bill DJ, Dourish CT. Behavioural and pharmacological characterisation of the elevated "zero-maze" as an animal model of anxiety. Psychopharmacology (Berl). 1994;116(1):56-64.

270. Crawley JN. Neuropharmacologic specificity of a simple animal model for the behavioral actions of benzodiazepines. Pharmacol Biochem Behav. 1981;15(5):695-9.

271. Crawley JN. Behavioral phenotyping of transgenic and knockout mice: experimental design and evaluation of general health, sensory functions, motor abilities, and specific behavioral tests. Brain Res. 1999;835(1):18-26.

272. File SE. Factors controlling measures of anxiety and responses to novelty in the mouse. Behav Brain Res. 2001;125(1-2):151-7.

273. Vogel JR, Beer B, Clody DE. A simple and reliable conflict procedure for testing anti-anxiety agents. Psychopharmacologia. 1971;21(1):1-7.

274. Pavlov PI. Conditioned reflexes: An investigation of the physiological activity of the cerebral cortex. Ann Neurosci. 2010;17(3):136-41.

275. Maren S, Quirk GJ. Neuronal signalling of fear memory. Nat Rev Neurosci. 2004;5(11):844-52.

276. Bogdanova OV, Kanekar S, D'Anci KE, Renshaw PF. Factors influencing behavior in the forced swim test. Physiol Behav. 2013;118:227-39.

277. Cryan JF, Mombereau C, Vassout A. The tail suspension test as a model for assessing antidepressant activity: review of pharmacological and genetic studies in mice. Neurosci Biobehav Rev. 2005;29(4-5):571-625.

278. Dulawa SC, Holick KA, Gundersen B, Hen R. Effects of chronic fluoxetine in animal models of anxiety and depression. Neuropsychopharmacology. 2004;29(7):1321-30.

279. Dulawa SC, Hen R. Recent advances in animal models of chronic antidepressant effects: the novelty-induced hypophagia test. Neurosci Biobehav Rev. 2005;29(4-5):771-83.

280. Willner P. Chronic mild stress (CMS) revisited: consistency and behaviouralneurobiological concordance in the effects of CMS. Neuropsychobiology. 2005;52(2):90-110. 281. Willner P. Validity, reliability and utility of the chronic mild stress model of depression: a 10-year review and evaluation. Psychopharmacology (Berl). 1997;134(4):31929.

282. Koolhaas JM, De Boer SF, De Rutter AJ, Meerlo P, Sgoifo A. Social stress in rats and mice. Acta Physiol Scand Suppl. 1997;640:69-72.

283. Yan HC, Cao X, Das M, Zhu XH, Gao TM. Behavioral animal models of depression. Neurosci Bull. 2010;26(4):327-37. 
284. Griffin WC, 3rd. Alcohol dependence and free-choice drinking in mice. Alcohol. 2014;48(3):287-93.

285. Carnicella S, Ron D, Barak S. Intermittent ethanol access schedule in rats as a preclinical model of alcohol abuse. Alcohol. 2014;48(3):243-52.

286. Simms JA, Steensland P, Medina B, Abernathy KE, Chandler LJ, Wise R, et al. Intermittent access to $20 \%$ ethanol induces high ethanol consumption in Long-Evans and Wistar rats. Alcohol Clin Exp Res. 2008;32(10):1816-23.

287. Spanagel R. Alcohol addiction research: from animal models to clinics. Best Pract Res Clin Gastroenterol. 2003;17(4):507-18.

288. Schuckit MA. Low level of response to alcohol as a predictor of future alcoholism. Am J Psychiatry. 1994;151(2):184-9.

289. Vanderschuren LJ, Everitt BJ. Behavioral and neural mechanisms of compulsive drug seeking. Eur J Pharmacol. 2005;526(1-3):77-88.

290. Wolffgramm J, Galli G, Thimm F, Heyne A. Animal models of addiction: models for therapeutic strategies? J Neural Transm (Vienna). 2000;107(6):649-68.

291. Vendruscolo LF, Roberts AJ. Operant alcohol self-administration in dependent rats: focus on the vapor model. Alcohol. 2014;48(3):277-86.

292. Vengeliene V, Celerier E, Chaskiel L, Penzo F, Spanagel R. Compulsive alcohol drinking in rodents. Addict Biol. 2009;14(4):384-96.

293. Spanagel R, Holter SM. Long-term alcohol self-administration with repeated alcohol deprivation phases: an animal model of alcoholism? Alcohol Alcohol. 1999;34(2):23143.

294. Batman AM, Miles MF. Translating Alcohol Research: Opportunities and Challenges. Alcohol Res. 2015;37(1):7-14.

$295 . \quad$ Prus AJ, James JR, Rosecrans JA. Conditioned Place Preference. In: Buccafusco JJ, editor. Methods of Behavior Analysis in Neuroscience. Frontiers in Neuroscience. 2nd ed. Boca Raton (FL)2009.

296. File SE. Factors controlling measures of anxiety and responses to novelty in the mouse. Behavioural brain research. 2001;125(1):151-7.

297. Gehlert DR, Cippitelli A, Thorsell A, Le AD, Hipskind PA, Hamdouchi C, et al. 3-(4-Chloro-2-morpholin-4-yl-thiazol-5-yl)-8-(1-ethylpropyl)-2,6-dimethyl-imidazo [1,2b]pyridazine: a novel brain-penetrant, orally available corticotropin-releasing factor receptor 1 antagonist with efficacy in animal models of alcoholism. J Neurosci. 2007;27(10):2718-26.

298. Ciccocioppo R, Stopponi S, Economidou D, Kuriyama M, Kinoshita H, Heilig $\mathrm{M}$, et al. Chronic treatment with novel brain-penetrating selective NOP receptor agonist MT7716 reduces alcohol drinking and seeking in the rat. Neuropsychopharmacology. 2014;39(11):2601-10.

299. Arnold JM, Roberts DC. A critique of fixed and progressive ratio schedules used to examine the neural substrates of drug reinforcement. Pharmacol Biochem Behav. 1997;57(3):441-7.

300. Richardson NR, Roberts DC. Progressive ratio schedules in drug selfadministration studies in rats: a method to evaluate reinforcing efficacy. J Neurosci Methods. 1996;66(1):1-11.

301. Gulotta KB, Byrne T. A progressive-duration schedule of reinforcement. Behav Processes. 2015;121:93-7.

302. Kotlinska J, Rafalski P, Biala G, Dylag T, Rolka K, Silberring J. Nociceptin inhibits acquisition of amphetamine-induced place preference and sensitization to stereotypy in rats. Eur J Pharmacol. 2003;474(2-3):233-9. 
303.

Sakoori K, Murphy NP. Central administration of nociceptin/orphanin FQ blocks the acquisition of conditioned place preference to morphine and cocaine, but not conditioned place aversion to naloxone in mice. Psychopharmacology (Berl). 2004;172(2):129-36.

304. Kalivas PW, McFarland K. Brain circuitry and the reinstatement of cocaineseeking behavior. Psychopharmacology (Berl). 2003;168(1-2):44-56.

305. Shaham Y, Shalev U, Lu L, De Wit H, Stewart J. The reinstatement model of drug relapse: history, methodology and major findings. Psychopharmacology (Berl). 2003;168(1-2):3-20.

306. Jenck F, Moreau JL, Martin JR, Kilpatrick GJ, Reinscheid RK, Monsma FJ, Jr., et al. Orphanin FQ acts as an anxiolytic to attenuate behavioral responses to stress. Proc Natl Acad Sci U S A. 1997;94(26):14854-8.

307. Koster A, Montkowski A, Schulz S, Stube EM, Knaudt K, Jenck F, et al. Targeted disruption of the orphanin FQ/nociceptin gene increases stress susceptibility and impairs stress adaptation in mice. Proc Natl Acad Sci U S A. 1999;96(18):10444-9.

308. Martin-Fardon R, Ciccocioppo R, Massi M, Weiss F. Nociceptin prevents stressinduced ethanol- but not cocaine-seeking behavior in rats. Neuroreport. 2000;11(9):1939-43.

309. Ciccocioppo R, Martin-Fardon R, Weiss F, Massi M. Nociceptin/orphanin FQ inhibits stress- and CRF-induced anorexia in rats. Neuroreport. 2001;12(6):1145-9.

310. Ciccocioppo R, Biondini M, Antonelli L, Wichmann J, Jenck F, Massi M. Reversal of stress- and CRF-induced anorexia in rats by the synthetic nociceptin/orphanin FQ receptor agonist, Ro 64-6198. Psychopharmacology (Berl). 2002;161(2):113-9.

311. Andero R, Brothers SP, Jovanovic T, Chen YT, Salah-Uddin H, Cameron M, et al. Amygdala-dependent fear is regulated by Oprl1 in mice and humans with PTSD. Sci Transl Med. 2013;5(188):188ra73.

312. Recker MD, Higgins GA. The opioid receptor like-1 receptor agonist Ro 64-6198 (1S,3aS-8-2,3,3a,4,5,6-hexahydro-1H-phenalen-1-yl-1-phenyl-1,3,8-triaza-spiro[4.5 ]decan4-one) produces a discriminative stimulus in rats distinct from that of a mu, kappa, and delta opioid receptor agonist cue. J Pharmacol Exp Ther. 2004;311(2):652-8.

313. Barak S, Carnicella S, Yowell QV, Ron D. Glial cell line-derived neurotrophic factor reverses alcohol-induced allostasis of the mesolimbic dopaminergic system: implications for alcohol reward and seeking. J Neurosci. 2011;31(27):9885-94.

314. Shen RY, Choong KC, Thompson AC. Long-term reduction in ventral tegmental area dopamine neuron population activity following repeated stimulant or ethanol treatment. Biol Psychiatry. 2007;61(1):93-100.

315. Weiss F, Parsons LH, Schulteis G, Hyytia P, Lorang MT, Bloom FE, et al. Ethanol self-administration restores withdrawal-associated deficiencies in accumbal dopamine and 5-hydroxytryptamine release in dependent rats. J Neurosci. 1996;16(10):3474-85.

316. Ahmed SH, Koob GF. Transition to drug addiction: a negative reinforcement model based on an allostatic decrease in reward function. Psychopharmacology (Berl). 2005;180(3):473-90.

317. Koob GF. Alcoholism: allostasis and beyond. Alcohol Clin Exp Res. 2003;27(2):232-43.

318. Meinhardt MW, Sommer WH. Postdependent state in rats as a model for medication development in alcoholism. Addict Biol. 2015;20(1):1-21.

319. Roberts AJ, Heyser CJ, Cole M, Griffin P, Koob GF. Excessive ethanol drinking following a history of dependence: animal model of allostasis. Neuropsychopharmacology. 2000;22(6):581-94.

320. Gilpin NW, Richardson HN, Cole M, Koob GF. Vapor inhalation of alcohol in rats. Curr Protoc Neurosci. 2008; Chapter 9:Unit 929. 
321. Green AS, Grahame NJ. Ethanol drinking in rodents: is free-choice drinking related to the reinforcing effects of ethanol? Alcohol. 2008;42(1):1-11.

322. Hodos W. Progressive ratio as a measure of reward strength. Science. 1961;134(3483):943-4.

323. Martin-Fardon R, Zorrilla EP, Ciccocioppo R, Weiss F. Role of innate and druginduced dysregulation of brain stress and arousal systems in addiction: Focus on corticotropinreleasing factor, nociceptin/orphanin FQ, and orexin/hypocretin. Brain Res. 2010;1314:14561.

324. Heyser CJ, Schulteis G, Koob GF. Increased ethanol self-administration after a period of imposed ethanol deprivation in rats trained in a limited access paradigm. Alcohol Clin Exp Res. 1997;21(5):784-91.

325. Collier G. Consummatory and instrumental responding as functions of deprivation. J Exp Psychol. 1962;64:410-4.

326. Bacchi F, Mathe AA, Jimenez P, Stasi L, Arban R, Gerrard P, et al. Anxiolyticlike effect of the selective neuropeptide Y Y2 receptor antagonist BIIE0246 in the elevated plus-maze. Peptides. 2006;27(12):3202-7.

327. Roy A, Pandey SC. The decreased cellular expression of neuropeptide Y protein in rat brain structures during ethanol withdrawal after chronic ethanol exposure. Alcohol Clin Exp Res. 2002;26(6):796-803.

328. Cippitelli A, Karlsson C, Shaw JL, Thorsell A, Gehlert DR, Heilig M. Suppression of alcohol self-administration and reinstatement of alcohol seeking by melaninconcentrating hormone receptor 1 (MCH1-R) antagonism in Wistar rats. Psychopharmacology (Berl). 2010;211(4):367-75.

329. Kowalski TJ, Spar BD, Weig B, Farley C, Cook J, Ghibaudi L, et al. Effects of a selective melanin-concentrating hormone 1 receptor antagonist on food intake and energy homeostasis in diet-induced obese mice. Eur J Pharmacol. 2006;535(1-3):182-91.

330. Rimondini R, Arlinde C, Sommer W, Heilig M. Long-lasting increase in voluntary ethanol consumption and transcriptional regulation in the rat brain after intermittent exposure to alcohol. FASEB J. 2002;16(1):27-35.

331. Rogers J, Wiener SG, Bloom FE. Long-term ethanol administration methods for rats: advantages of inhalation over intubation or liquid diets. Behav Neural Biol. 1979;27(4):466-86.

332. Morganstern I, Chang GQ, Chen YW, Barson JR, Zhiyu Y, Hoebel BG, et al. Role of melanin-concentrating hormone in the control of ethanol consumption: Region-specific effects revealed by expression and injection studies. Physiol Behav. 2010;101(4):428-37.

333. Schmid CL, Bohn LM. Physiological and pharmacological implications of betaarrestin regulation. Pharmacol Ther. 2009;121(3):285-93.

334. Toll L, Bruchas MR, Calo G, Cox BM, Zaveri NT. Nociceptin/Orphanin FQ Receptor Structure, Signaling, Ligands, Functions, and Interactions with Opioid Systems. Pharmacol Rev. 2016;68(2):419-57. 


\section{Scientific Articles}

The articles associated with this thesis have been removed for copyright reasons. For more details about these see:

http://urn.kb.se/resolve?urn=urn:nbn:se:liu:diva-139884 